\title{
The role of electrical anisotropy in magnetotelluric responses: From modelling and dimensionality analysis to inversion and interpretation
}

\author{
Anna Martí \\ GEOMODELS Research Institute, Departament de Geodinàmica i Geofísica, \\ Facultat de Geologia. Universitat de Barcelona. C/ Martí Franquès s/n. 08028 \\ Barcelona. Spain. \\ E-mail: annamarti@ub.edu
}

\begin{abstract}
The study of electrical anisotropy in the Earth, defined as the electrical conductivity varying with orientation, has experienced important advances in the last years regarding the investigation of its origins, how to identify and model it, and how it can be related to other parameters, such as seismic and mechanical anisotropy. This paper aims to provide a theoretical background and to be a review of the current state of the art of electrical anisotropy using electromagnetic methods in the frequency domain, focusing mainly on magnetotellurics. The aspects that will be considered are the modelling of the electromagnetic fields with anisotropic structures, the analysis of their responses to identify these structures, and how to properly use these responses in inversion and interpretation. Also, an update on the most recent case studies involving anisotropy is provided.
\end{abstract}

Keywords: Electrical anisotropy, magnetotelluric responses, dimensionality analysis, inversion 


\section{Introduction}

Within the study of the electrical properties of the Earth's interior, electrical anisotropy, for which electrical conductivity depends on orientation, can be recognized using electromagnetic (EM) methods, although its proper identification, characterization and interpretation are currently significantly debated.

Among EM methods, the magnetotelluric (MT) method is based on the measurement of time variations of the horizontal components of the natural electric and magnetic fields at the Earth's surface. The MT responses are defined in terms of the impedance tensor, from which apparent resistivity and phases can be obtained, and the geomagnetic transfer function, or Tipper, if the vertical component of the magnetic field is also recorded. It is in the responses themselves, during the dimensionality analysis of a full dataset or during the process of modelling or inversion of the data, that hints of anisotropy can be recognized. However, due to the non-uniqueness of the method (at least for anisotropic structures) and the lack of exact data (i.e. errors and insufficient data), anisotropy cannot always be identified. This can happen when anisotropy has the same orientation as the structures in question, occurs within 3D structures, or is confused or hidden within other effects such as galvanic distortion or current channelling.

The goal of this paper is to present a review on different works as a guide to understanding: a) how anisotropy affects the magnetotelluric responses, b) how to identify its footprint; and c) how to proceed in real situations, with modelling codes and strategies for inversion. Additionally, a summary with the most recent case studies involving electrical anisotropy will be provided.

The previous review on electrical anisotropy by Wannamaker (2005) focused on the causes for the electrical anisotropy observed using electromagnetic methods, with examples from different continental settings. Baba (2005) complemented it with examples from marine tectonics. Hence, studies related to the origins of the electrical anisotropy will be commented on briefly, and applications in specific areas will be presented at the end. 
Electrical anisotropy in the Earth observed using EM methods can have a microscopic origin (e.g. preferred orientations within crystals) or it can be a macroscopic effect due to the inability of the EM methods to resolve oriented structures (layering or lamination) smaller than the averaged volume (Weidelt 1999). The causes of anisotropy at different depths can be preferred orientation of fracture porosity, fluidized, melt-bearing or graphitized shear zones, lithologic layering, oriented heterogeneity, or hydrous defects within shear aligned olivine crystals (Wannamaker 2005). In marine settings, the conductivity of the sediments can be anisotropic due to grain-scale anisotropy in shales owing to mineral alignment, or macro-anisotropic due to interbedding of layers of different conductivities, for which the vertical resistivity is generally higher than the horizontal (Key 2012).

Currently the interpretation of electromagnetic responses showing anisotropy at long periods is accompanied by laboratory measurements of the electrical conductivity simulating crustal or mantle conditions (detailed reviews can be found in Nover 2005; Yoshino 2010; Pommier 2012). However, regarding the origins of the high electrical conductivity and strong anisotropy observed in the Earth's mantle using MT, there is currently strong debate. This is because MT observations infer anisotropy coefficients much higher (up to two orders of magnitude, e.g. Gatzemeier and Moorkamp 2005) than laboratory measurements. Some laboratory data (Wang et al. 2006) show that this might be caused by hydrous olivine, enhanced by other mechanisms (Gatzemeier and Tommasi 2006), perhaps at grain boundaries (Simpson and Tommasi 2005). Alternatively, studies from Yoshino et al. (2006), Gaillard et al. (2008) or Caricchi et al. (2011) point to partial melting.

Wannamaker (2005) also describes papers that relate electrical anisotropy to seismic anisotropy. Even if the relationship between them is not clear (although this is under study, e.g. Carcione et al. 2007; Jones et al. 2009a), they point to a common origin. In tectonic continental settings, the importance of anisotropy (electrical, seismic or mechanical) is recognized in the introduction of Eaton and Jones (2006) to the special volume on the study of continental tectonic fabrics 
using anisotropy constraints. Among papers on seismic and mechanical anisotropy, the volume contains articles with case studies using electromagnetic methods combined with seismic SKS splitting analysis and ones more specific to MT methodology. Although this review is not focused on comparing seismic and electrical anisotropy, some of those papers will be discussed.

After a brief introduction on the theoretical basis and terms related to electrical anisotropy, I will present a review of modelling and inversion codes that have been developed over the years. I will then provide the formulation of Maxwell's equations in anisotropic settings with the solutions for the simplest scenarios. Subsequently, I will comment on the features of these responses as described by different authors, and on papers regarding the removal of distortion, and finally, on dimensionality analysis. The following section will focus on new inversion codes, how they have been applied, and on work done analyzing several aspects of inversion of anisotropic data using different types of codes. I will complement the review with a brief update of the most recent case studies involving anisotropy.

\section{Understanding the responses}

\subsection{Mathematical and physical background}

In an anisotropic medium, the electrical conductivity at each point $(x, y, z)$ is defined by the conductivity tensor:

$$
\hat{\sigma}(x, y, z)=\left[\begin{array}{ccc}
\sigma_{x x} & \sigma_{x y} & \sigma_{x z} \\
\sigma_{y x} & \sigma_{y y} & \sigma_{y z} \\
\sigma_{z x} & \sigma_{z y} & \sigma_{z z}
\end{array}\right],(1)
$$

where $\mathrm{x}, \mathrm{y}, \mathrm{z}$ are defined herein in Cartesian coordinates, with $\mathrm{x}$ towards the north, $\mathrm{y}$ towards the east and $\mathrm{z}$ directly down, with origin at any point on the surface of the Earth. The interpretation of the non-diagonal elements denotes the deviation of the anisotropy axes from the Cartesian axes (Fig. 1). 
The conductivity tensor has two properties:

- It is a positive-definite matrix $\left(\vec{z}^{T} \cdot \vec{\sigma} \cdot \vec{z}>0\right.$, for all non-zero vectors $z$ with real entries). This is required to ensure that the specific energy dissipation $1 / 2 \vec{E}^{*} \cdot \vec{O} \cdot \vec{E}$ (dissipated energy per unit volume and time = integral of the normal component of the Poynting vector over a closed surface, Weidelt 2007) is nonnegative (Weidelt 1999). This product would be zero in the air, where the tensor is assumed to have all elements $=0$. As a consequence, the angle between $\boldsymbol{j}$ and $\boldsymbol{E}$ ( $\vec{j}=\hat{\sigma} \cdot \vec{E}$ ) must be less than $90^{\circ}$.

- It is symmetric. Whenever the magnetic field does not play a role in the conduction process (e.g. pure ohmic conduction, as opposed to Hall currents), the tensor is symmetric (a proof of that can be found in Dekker and Hastie 1980 (APPENDIX C)). Therefore, when I refer to only one of the non-diagonal components of the conductivity tensor, the same is assumed for the symmetric component.

Using Euler's elementary rotations the conductivity tensor can be diagonalized and its principal directions obtained, namely the strike, dip and slant anisotropy angles ( $\alpha_{S}$ around z-axis, $\alpha_{D}$ around x'-axis and $\alpha_{L}$ around z''-axis) (Fig. 1), although other parametrizations might apply. Hence, the conductivity tensor $\left(\hat{\sigma}^{\prime}\right)$ can be specified by six parameters: the three conductivity components along the principal directions $\left(\sigma_{x x}{ }^{\prime}, \sigma_{y y}{ }^{\prime}, \sigma_{z z}{ }^{\prime}\right.$ or, simply, $\sigma_{x}, \sigma_{y}$ and $\left.\sigma_{z}\right)$ and their corresponding angles:

$$
\hat{\sigma}^{\prime}(x, y, z)=R \cdot \hat{\sigma} \cdot R^{T}=\left[\begin{array}{ccc}
\sigma_{x} & 0 & 0 \\
0 & \sigma_{y} & 0 \\
0 & 0 & \sigma_{z}
\end{array}\right],(2)
$$

where $R$ is the rotation matrix resulting from the three elementary rotations. 
Depending on the number of different conductivity values in the principal directions, the anisotropy is referred to as biaxial (or triaxial, $\sigma_{x} \neq \sigma_{y} \neq \sigma_{z}$ ) or uniaxial $\left(\sigma_{x}=\sigma_{y}, \sigma_{x}=\sigma_{z}\right.$ or $\left.\sigma_{y}=\sigma_{z}\right)$. As for the geometry of the anisotropy (notation from Li 2002), one can have azimuthal (or horizontal) anisotropy when only the strike angle $\alpha_{S}$ is non-zero; dipping anisotropy, when the only non-zero angle is $\alpha_{D}$; or vertical (or transverse) anisotropy when all the angles are null and the tensor is diagonal in the measuring reference frame. Table 1 summarizes these cases, with particular cases for uniaxial anisotropy.

One parameter commonly used to characterize the amount of anisotropy is the anisotropy ratio, defined as the ratio between the maximum and minimum resistivity values $\left(\rho_{i}=1 / \sigma_{j}\right)$ of the principal directions: $\rho_{\max } / \rho_{\min }$ (which is equivalent to $\sigma_{\max } / \sigma_{\min }$ ), although other definitions might hold as well (e.g. square root). For completeness, anisotropy can also be described by the anisotropy ellipsoid parameters, anisotropy degree, $P$ and anisotropy shape, $S$ (Jelínek, 1981).

Anisotropy can be embedded within 1D, 2D or 3D structures; in each case affecting the EM responses in a different way. These situations are referred to as $1 \mathrm{D}$ anisotropic, 2D anisotropic and 3D anisotropic models. Before reviewing the magnetotelluric responses for these types of models, in the next section I will present a compilation of the different codes on modelling and inversion that have been introduced over the years.

\subsection{Outline on the progress of modelling and inversion of MT data considering anisotropic structures}

I will start by going back almost half a century. At that time, the magnetotelluric method was in its infancy and either the conductivity and the frequency dependent impedance and apparent resistivities were defined as scalars. In general, the word "anisotropy" was used to illustrate any lateral change in conductivity, which occurred when the measured $E_{x} / H_{y}$ differed from $E_{y} / H_{x}$. Neves (1957) introduced the impedance tensor $\underline{Z}(\omega)$, and the related "tensorial apparent resistivities" (or apparent resistivities). The impedance tensor does not depend on the magnitude 
and polarization of the source fields and theoretically only two measurements at a given frequency is sufficient to determine the full tensor.

Mann (1965) introduced the concept of electrical anisotropy in magnetotellurics and represented the conductivity as a $3 \times 3$ tensor. In his paper he formulated the mathematical problem of an anisotropic half-space, assuming a flat incident EM wave at the surface. According to his development, the vertical fields $H_{z}$ and $E_{z}$ are zero at both the surface and within the half space (although $E_{z}$ within a medium is not zero, in general) and there are two modes propagating downwards through the medium, with wave numbers depending on the values of the principal horizontal conductivities. The author points out the importance of using $\underline{Z}$ and tensorial apparent resistivities instead of scalar ones, otherwise the solution is dependent on the polarization of the fields. Compared to the problems of a vertical fault and of a sinusoidal interface, which solutions converge at short periods; the anisotropic model counterpart does not converge to these other solutions, and hence it shows a different nature than the responses of isotropic media.

In subsequent years several authors formulated the solution of the forward problem in different 1D scenarios (O’Brien and Morrison 1967; Praus and Petr 1969; Sinha 1969; Reddy and Rankin 1971; Negi and Saraf 1972, 1973; Loewenthal and Landisman 1973; Abramovici 1974; Shoham and Loewenthal 1975; Dekker and Hastie 1980). More recently, codes were provided by Pek and Santos (2002) and Yin (2006) (Marine MT). The solution at the surface is characterized by the impedance tensor having diagonal components with equal amplitude but opposite signs, and zero vertical magnetic field (hence the Tipper vector $\vec{T}$ is null). Similar problems were solved in anisotropic 1D media for CSTMT (Controlled Source Tensor MT, Li and Pedersen 1991; Li and Pedersen 1992), in which case, because of working in near and intermediate field, the tipper vector is non-zero and provides valuable information; CSAMT (Controlled Source Audio MT, Yin and Maurer 2001) and DC (direct current, Yin and Weidelt 1999).

Abramovici and Shoham (1977) proposed the solution of the inverse problem in simple 1D anisotropic media. Yin (2003) proved that (as in DC, Yin 2000) the 
solution of the inverse problem in MT is inherently non-unique (as opposed to isotropic 1D inversion, which, theoretically, with accurate and complete data, is unique). Due to the fact that currents flow horizontally, the vertical conductivity cannot be resolved. This is reflected in the work of Pek and Santos (2002), which presents the forward responses and parametric sensitivities for 1D anisotropic media using an equivalent azimuthal anisotropy conductivity tensor that is characterized by 3 parameters instead of 6 . Li et al. (2000) presented an inversion approach to invert CSTMT data for azimuthal anisotropy in a 1D layered earth, including electric and magnetic distortion in the modelling parameters, and simultaneously fitted impedances and Tipper, which was not possible using isotropic models. Linde and Pedersen (2004) presented a similar approach with an application to radiomagnetotelluric data (RMT). Pek and Santos (2006) presented a 1D MT inversion code based on the Occam algorithm (Constable et al. 1987). Novel codes were developed by Roux et al. (2011) and Mandolesi and Jones (2012a), with joint inversion of magnetotelluric data and surface-wave dispersion curves for 1D anisotropic structures.

The first steps towards 2D were taken by Reddy and Rankin (1975) who computed the MT responses of a laterally inhomogeneous anisotropic media, specifically that of a dyke, with azimuthal anisotropy both inside and outside the dyke. They obtained solutions for $\underline{Z}$ and $\vec{T}$, using two arbitrary source $\vec{H}$ fields. The analysis of the results shows that the "additional impedances" $\left(Z_{x x}\right.$ and $\left.Z_{y y}\right)$ are non-zero along the structural direction and that the skew ( $\left.\left(Z_{x x}+Z_{y y}\right) /\left(Z_{x y}-Z_{y x}\right)\right)$ and ellipticity $\left(\left(Z_{x x}-Z_{y y}\right) /\left(Z_{x y}+Z_{y x}\right)\right)$ parameters do not vanish, as a consequence of the anisotropy not being along the measuring axes. The non-vanishing of $H_{z}$ is due to the lateral inhomogeneity rather than the anisotropy itself (as anisotropies alone do not produce a vertical magnetic field). Saraf et al. (1986) presented the solution for the TM mode of structures with vertical anisotropy, and Osella and Martinelli (1993), for stratified models with smooth irregular layers, with the anisotropy along the measuring directions.

By far, the most known and used code is the one of Pek and Verner (1997), which computes the MT responses at the surface (EM fields $\underline{Z}$ and $\vec{T}$ ) for $2 \mathrm{D}$ models with arbitrary anisotropy (any orientations), using the finite-difference method. 
This is a reliable code that the authors have made available to the MT community. Li (2002) tackles the same problem by computing the horizontal fields and transfer functions using finite elements, and the computation of the vertical magnetic field was also implemented in Brasse et al. (2009). Li and Pek (2008) presented an adaptive finite element solver for the MT forward problem in 2D generally anisotropic media, aiming at arbitrarily shaped structural boundaries, rough topographies and bathymetries, such as table mountains, sea hills and volcanoes. Li et al. (2012) simulated the CSEM field responses from 2D models with arbitrary anisotropy using an adaptive finite element approach. More recently, Qin et al. (2013) derived the quasi-analytic solution for the 2D magnetotelluric fields on an axially anisotropic infinite fault.

The steps done towards the inversion in 2D anisotropic media, with significant progress, include the following works: Li et al. (2003) presented a methodology to invert all the components of the apparent resistivity and phase, and obtain 6 anisotropic parameters (3 principal conductivities and 3 angles) for each cell, within a 2D structure. Mackie (2002) and Baba et al. (2006) developed an inversion code that inverts TE and TM data, considering azimuthal anisotropy aligned with the 2D structure, based on the code of Rodi and Mackie (2001). Pek et al. (2011) (and J. Pek, pers. comm.) have a preliminary version of a 2D inversion code which considers anisotropy in any direction. Using the Pek and Verner (1997) 2D code to solve the forward responses, Mandolesi and Jones (2012b) are working on an inversion code, based on a classical LevenbergMarquardt strategy. Despite some promising results from their synthetic example, the authors show the difficulty of correctly fitting $\sigma_{z z}$. Similarly, Chen and Weckmann (2012) presented an inversion code that adds an additional term with the variation of the conductivity tensor elements in the penalty function. Montahaie and Oskooi (2012) implemented an inversion method to obtain 1D and 2D azimuthally anisotropic models based on artificial neural networks; and Plotkin (2012) described a procedure to invert laterally heterogeneous anisotropic responses collected using a dense synchronous 2D MT array, which improves the reconstruction of the full conductivity tensor.

In 3D, Martinelli and Osella (1997) presented a solution for 3D models with vertical anisotropy using the Rayleigh-Fourier technique. Weidelt (1999) 
formulated the forward solution of the MT problem for a 3D anisotropic medium using a staggered-grid finite-difference method, and points out that anisotropic models cannot explain data that cannot be interpreted by isotropic 3D models of arbitrary complexity (which will be discussed later in the text). Pankratov et al. (1997) developed a technique, based on a Modified Neumann Series, to solve Maxwell's equation for 3D arbitrary anisotropy. Wang and Fang (2001) and Weiss and Newman $(2002,2003)$ presented the general solution for a fully 3D anisotropic earth, using staggered-grid finite differences, and with examples from induction logging. Within multi-component induction logging several forward 3D codes incorporating anisotropy have been presented (e.g. Davydycheva et al. 2003; Hou et al. 2006; Davydycheva and Wang 2011; Everett 2012 and references therein). In DC resistivity modelling, Li and Spitzer (2005) presented a 3D code for arbitrary anisotropy using finite elements.

In marine controlled source electromagnetic (CSEM) several forward (e.g. Edwards 2005 and references therein; Løseth and Ursin 2007; Kong et al. 2008; Li and Dai 2011) and inverse (e.g. Ramananjaona and MacGregor 2010) formulations and codes considering anisotropy have been developed. Newman et al. (2010) presented a code that solves the 3D inverse problem for structures with transverse anisotropy, which is the situation observed generally in geologic basins. In MT per se there are no 3D anisotropic inversion codes freely available yet, but in marine contexts CSEM and MT data have proven to be complementary to image sedimentary anisotropic structures (e.g. Ramananjaona et al. 2011). In this sense, Sasaki (2011) presented a 3D code which jointly inverts CSEM and MT marine data.

Independently of these anisotropic codes, several authors have approached the problem by modelling macro-anisotropy using isotropic codes, either in 1D (Padilha et al. 2006), or 3D (e.g. Leibecker et al. 2002; Gatzemeier and Moorkamp 2005), as a succession of alternate lamellae of different conductivities aligned in certain directions. 


\subsection{Magnetotelluric responses from anisotropic models}

In the quasi-stationary approximation, in absence of charges, and considering a harmonic $e^{-i \omega t}$ time dependence of the electric and magnetic fields (following the notation of Pek and Verner 1997), Maxwell's equations can be expressed in the frequency domain as:

$$
\begin{gathered}
\vec{\nabla} \times \vec{E}=i \omega \mu_{0} \vec{H},(3 \mathrm{a}) \\
\vec{\nabla} \times \vec{H}=\hat{\sigma} \cdot \vec{E},(3 \mathrm{~b})
\end{gathered}
$$

where the value of magnetic permeability $(\mu)$ is considered equal to the value in a vacuum $\left(\mu_{0}\right)$. I implicitly assume the dependence of the electric and magnetic fields on frequency $(\omega)$.

\section{1) $1 D$ anisotropic models}

In a $1 \mathrm{D}$ anisotropic Earth, where the conditions $\partial / \partial x=\partial / \partial y=0$ can be applied, Maxwell's equations ( $3 a$ ) and ( $3 b)$ reduce to:

$$
\begin{gathered}
\frac{\partial E_{x}}{\partial z}=i \omega \mu_{0} H_{y},(4 \mathrm{a}) \\
\frac{\partial E_{y}}{\partial z}=-i \omega \mu_{0} H_{x},(4 \mathrm{~b}) \\
H_{z}=0,(4 \mathrm{c})
\end{gathered}
$$

and

$$
\begin{gathered}
\frac{\partial H_{y}}{\partial z}=-\sigma_{x x} E_{x}-\sigma_{x y} E_{y}-\sigma_{x z} E_{z},(5 \mathrm{a}) \\
\frac{\partial H_{x}}{\partial z}=\sigma_{y x} E_{x}+\sigma_{y y} E_{y}+\sigma_{y z} E_{z},(5 \mathrm{~b})
\end{gathered}
$$




$$
\sigma_{z x} E_{x}+\sigma_{z y} E_{y}+\sigma_{z z} E_{z}=0
$$

As a common procedure, we take the derivative of Eqs. (4a) and (4b), and eliminate the magnetic fields by substituting their derivatives in (5a) and (5b), obtaining a system of $2^{\text {nd }}$ order differential equations for the horizontal electric fields, which has the form of coupled pendulums equations,

$$
\begin{aligned}
& \frac{\partial^{2} E_{x}}{\partial z^{2}}+i \omega \mu_{0}\left(A_{x x} E_{x}+A_{x y} E_{y}\right)=0,(6 \mathrm{a}) \\
& \frac{\partial^{2} E_{y}}{\partial z^{2}}+i \omega \mu_{0}\left(A_{y x} E_{x}+A_{y y} E_{y}\right)=0,(6 \mathrm{~b})
\end{aligned}
$$

with:

$$
\begin{gathered}
A_{x x}=\sigma_{x x}-\frac{\sigma_{x z} \sigma_{z x}}{\sigma_{z z}}=\sigma_{x x}-\frac{\sigma_{x z}^{2}}{\sigma_{z z}},(7 \mathrm{a}) \\
A_{x y}=\sigma_{x y}-\frac{\sigma_{x z} \sigma_{z y}}{\sigma_{z z}},(7 \mathrm{~b}) \\
A_{y x}=\sigma_{x y}-\frac{\sigma_{y z} \sigma_{z x}}{\sigma_{z z}},(7 \mathrm{c}) \\
A_{y y}=\sigma_{y y}-\frac{\sigma_{y z} \sigma_{z y}}{\sigma_{z z}}=\sigma_{x x}-\frac{\sigma_{y z}^{2}}{\sigma_{z z}} .(7 \mathrm{~d})
\end{gathered}
$$

From Eqs. (6a) and (6b) it can be seen that EM fields at the surface will depend on 3 parameters: $A_{x x}, A_{x y}\left(=A_{y x}\right)$ and $A_{y y}$. Whatever is the form of the conductivity tensor, the value of $\sigma_{z z}$ is always linked to either $\sigma_{x z}$ or $\sigma_{y z}$, which means that the solutions will depend on $\sigma_{z z}$ only in the case of dipping anisotropy. Hence, following Pek and Santos(2002), in a 1D anisotropic medium, the conductivity tensor at each layer is equivalent to an azimuthally anisotropic layer, with components $A_{x x}, A_{x y}\left(=A_{y x}\right)$ and $A_{y y}$, which can be diagonalised with principal horizontal conductivities $A_{1}$ and $A_{2}$, and effective anisotropic strike $\beta_{S}$. 
The solutions of Eqs. (6a) and (6b) in each layer are two pairs of propagating waves, each with a $e^{+k z}$ and $e^{-k z}$ dependence (as would happen in a 2D isotropic earth), and a different wave number $k\left(k_{1}\right.$ and $\left.k_{2}\right)$ directly related to the values of $A_{i j}$ (Pek and Santos 2002). Hence, two pairs of downgoing and upgoing waves exist in a generally anisotropic layer, the first one "slow" (related to the greater of the two effective conductivities), and the other "fast" (the one in the plane of the lower conductivity direction) (Pek 2009). The different values of $k$ mean that for the same frequency, different depths are being investigated for each wave number.

In an N-layered media, the impedance tensor can be obtained by propagating the relationships between EM field components from the bottom basement to the surface, applying the corresponding boundary conditions (either the full impedance tensor or the Ricatti equations as suggested by Kováčiková and Pek 2002, which significantly simplify the algebra involved). The impedance tensor has the form (e.g. Jones, 2012a):

$$
\underline{Z}_{1 D-a n i s}=\left[\begin{array}{cc}
Z_{x x} & Z_{x y} \\
Z_{y x} & -Z_{x x}
\end{array}\right]
$$

The condition of the zero trace of the impedance tensor $\left(Z_{x x}+Z_{y y}=0\right)$ is the same as in a 2D model (Kováčiková and Pek 2002). However, it is not necessarily diagonalizable by rotation if more than one anisotropy strike occurs in different layers. The vertical magnetic field at any point in the medium and at the surface is zero (Eq. 4c), hence the tipper vector is $\vec{T}=0$.

For the particular case of an anisotropic half-space (assuming the effective azimuthal anisotropy characterised by $A_{1}, A_{2}$ and $\beta_{S}$ ), the impedance tensor is related to the components of the conductivity tensor by the expression:

$$
Z_{\text {half-space-anis }}=-\frac{1}{2}\left[\begin{array}{cc}
-\left(\varsigma_{1}-\varsigma_{2}\right) \sin 2 \beta_{S} & \left(\varsigma_{1}+\varsigma_{2}\right)+\left(\varsigma_{1}-\varsigma_{2}\right) \cos 2 \beta_{S} \\
-\left(\varsigma_{1}+\varsigma_{2}\right)+\left(\varsigma_{1}-\varsigma_{2}\right) \cos 2 \beta_{S} & \left(\varsigma_{1}-\varsigma_{2}\right) \sin 2 \beta_{S}
\end{array}\right],
$$


with $\varsigma_{i}=\sqrt{i \omega \mu_{0} / A_{i}}$. Along the measuring directions $\left(\beta_{S}=0\right)$ the tensor becomes anti-diagonal, as in a 2D case. Thus, in the simplest case of azimuthal anisotropy, along the measuring $x$ and $y$ axes:

$$
\underline{Z}_{\text {half-space-azim-anis }}=\left[\begin{array}{cc}
0 & -\varsigma_{1} \\
\varsigma_{2} & 0
\end{array}\right]=\sqrt{\frac{\omega \mu_{0}}{2}}\left[\begin{array}{cc}
0 & -1 / \sqrt{\sigma_{x}} \\
1 / \sqrt{\sigma_{y}} & 0
\end{array}\right](1-i) .(10)
$$

For a 1D medium with one anisotropic layer (see model example and responses in Fig. 2), the apparent resistivities and phases computed at one site resemble those from a 2D model with identical, but not null, diagonal responses (unless the anisotropic strike is null). In real data, this is usually observed as a constant phase splitting between two modes at different sites. These responses can be distinguished from those of a 2D isotropic one because of the absence of tipper and the repetition of the responses at different sites. In a 2D model, if measurements were made along the strike, the impedances would be identical as well, but the tipper would have a component perpendicular to the strike direction.

\section{2) $2 D$ anisotropic models}

In a 2D anisotropic earth (following the notation of Pek and Verner 1997), considering the strike along the $\mathrm{x}$ direction, the condition $\partial / \partial x=0$ can be applied, and Maxwell's equations (3a) and (3b) become:

$$
\begin{gathered}
\frac{\partial E_{z}}{\partial y}-\frac{\partial E_{y}}{\partial z}=i \omega \mu_{0} H_{x},(11 \mathrm{a}) \\
\frac{\partial E_{x}}{\partial z}=i \omega \mu_{0} H_{y},(11 \mathrm{~b}) \\
-\frac{\partial E_{x}}{\partial y}=i \omega \mu_{0} H_{z},(11 \mathrm{c})
\end{gathered}
$$

and 


$$
\begin{gathered}
\frac{\partial H_{z}}{\partial y}-\frac{\partial H_{y}}{\partial z}=\sigma_{x x} E_{x}+\sigma_{x y} E_{y}+\sigma_{x z} E_{z},(12 \mathrm{a}) \\
\frac{\partial H_{x}}{\partial z}=\sigma_{y x} E_{x}+\sigma_{y y} E_{y}+\sigma_{y z} E_{z},(12 \mathrm{~b}) \\
-\frac{\partial H_{x}}{\partial y}=\sigma_{z x} E_{x}+\sigma_{z y} E_{y}+\sigma_{z z} E_{z} \cdot(12 \mathrm{c})
\end{gathered}
$$

Taking $2^{\text {nd }}$ derivatives and some algebra, we obtain two coupled second-order partial differential equations for $E_{x}$ and $H_{x}$ :

$$
\begin{gathered}
\frac{\partial^{2} E_{x}}{\partial y^{2}}+\frac{\partial^{2} E_{x}}{\partial z^{2}}+i \omega \mu_{0}\left(\sigma_{x x}+\sigma_{z x} A+\sigma_{x y} B\right) E_{x}+i \omega \mu_{0} A \frac{\partial H_{x}}{\partial y}-i \omega \mu_{0} B \frac{\partial H_{x}}{\partial z}=0, \\
\quad \frac{\partial}{\partial y}\left(\frac{\sigma_{y y}}{D} \frac{\partial H_{x}}{\partial y}\right)+\frac{\partial}{\partial z}\left(\frac{\sigma_{z z}}{D} \frac{\partial H_{x}}{\partial z}\right)+\frac{\partial}{\partial y}\left(\frac{\sigma_{y z}}{D} \frac{\partial H_{x}}{\partial z}\right)+\frac{\partial}{\partial z}\left(\frac{\sigma_{y z}}{D} \frac{\partial H_{x}}{\partial y}\right),(13 \mathrm{~b}) \\
+i \omega \mu_{0} H_{x}-\frac{\partial\left(A E_{x}\right)}{\partial y}-\frac{\partial\left(B E_{x}\right)}{\partial z}=0
\end{gathered}
$$

with:

$$
\begin{gathered}
A=\frac{\sigma_{x y} \sigma_{y z}-\sigma_{x z} \sigma_{y y}}{D},(14 \mathrm{a}) \\
B=\frac{\sigma_{x z} \sigma_{y z}-\sigma_{x y} \sigma_{z z}}{D},(14 \mathrm{~b}) \\
D=\sigma_{y y} \sigma_{z z}-\sigma_{y z}^{2} .(14 \mathrm{c})
\end{gathered}
$$

In the isotropic case: equations become decoupled into two distinct modes:

$$
\frac{\partial^{2} E_{x}}{\partial y^{2}}+\frac{\partial^{2} E_{x}}{\partial z^{2}}+i \omega \mu_{0} \sigma E_{x}=0 \text { (TE mode), (15a) }
$$




$$
\frac{\partial^{2} H_{x}}{\partial y^{2}}+\frac{\partial^{2} H_{x}}{\partial z^{2}}+i \omega \mu_{0} H_{x}=0(\text { TM mode }) .(15 b)
$$

For vertical and dipping anisotropy, the equations can be decoupled as well: Vertical anisotropy (e.g. Baba et al. 2006) $\left(A=0, B=0\right.$ and $\left.D=\sigma_{y y} \sigma_{z z}\right)$ :

$$
\begin{gathered}
\frac{\partial^{2} E_{x}}{\partial y^{2}}+\frac{\partial^{2} E_{x}}{\partial z^{2}}+i \omega \mu_{0} \sigma_{x x} E_{x}=0,(16 \mathrm{a}) \\
\frac{\partial}{\partial y}\left(\frac{1}{\sigma_{z z}} \frac{\partial H_{x}}{\partial y}\right)+\frac{\partial}{\partial z}\left(\frac{1}{\sigma_{y y}} \frac{\partial H_{x}}{\partial z}\right)+i \omega \mu_{0} H_{x}=0
\end{gathered}
$$

Dipping anisotropy $\left(A=0, B=0\right.$ and $\left.D=\sigma_{y y} \sigma_{z z}-\sigma_{y z}^{2}\right)$ :

$$
\begin{gathered}
\frac{\partial^{2} E_{x}}{\partial y^{2}}+\frac{\partial^{2} E_{x}}{\partial z^{2}}+i \omega \mu_{0} \sigma_{x x} E_{x}=0,(17 \mathrm{a}) \\
\frac{\partial}{\partial y}\left(\frac{\sigma_{y y}}{D} \frac{\partial H_{x}}{\partial y}\right)+\frac{\partial}{\partial z}\left(\frac{\sigma_{z z}}{D} \frac{\partial H_{x}}{\partial z}\right)+\frac{\partial}{\partial y}\left(\frac{\sigma_{y z}}{D} \frac{\partial H_{x}}{\partial z}\right)+\frac{\partial}{\partial z}\left(\frac{\sigma_{y z}}{D} \frac{\partial H_{x}}{\partial y}\right)+i \omega \mu_{0} H_{x}=0
\end{gathered}
$$

The solutions of Eqs. (16a) and (17a) would be the same as in a medium with scalar conductivity $\sigma=\sigma_{x x}$ whereas the ones of (16b) and (17b) would result from a combination of the rest of the conductivity values in the different directions.

In the case of azimuthal anisotropy: $\sigma(x, y, z)=\left[\begin{array}{ccc}\sigma_{x x} & \sigma_{x y} & 0 \\ \sigma_{y x} & \sigma_{y y} & 0 \\ 0 & 0 & \sigma_{z z}\end{array}\right], A=0$, $\mathrm{B}=-\sigma_{x y} / \sigma_{y y}, D=\sigma_{y y} \sigma_{z z}$ and the equations cannot be decoupled but have simpler expressions than in the general case (13a) and (13b):

$$
\frac{\partial^{2} E_{x}}{\partial y^{2}}+\frac{\partial^{2} E_{x}}{\partial z^{2}}+i \omega \mu_{0}\left(\sigma_{x x}-\frac{\sigma_{x y}^{2}}{\sigma_{y y}}\right) E_{x}+i \omega \mu_{0} \frac{\sigma_{x y}}{\sigma_{y y}} \frac{\partial H_{x}}{\partial z}=0, \text { (18a) }
$$




$$
\frac{\partial}{\partial y}\left(\frac{1}{\sigma_{z z}} \frac{\partial H_{x}}{\partial y}\right)+\frac{\partial}{\partial z}\left(\frac{1}{\sigma_{y y}} \frac{\partial H_{x}}{\partial z}\right)+i \omega \mu_{0} H_{x}+\frac{\partial}{\partial z}\left(\frac{\sigma_{x y}}{\sigma_{y y}} E_{x}\right)=0
$$

and the solutions must be sought at a higher computational cost.

In any $2 \mathrm{D}$ anisotropic case, once $E_{x}$ and $H_{x}$ are determined at the surface, the rest of the field components can be computed, through differentiation (or integration):

$$
\begin{gathered}
H_{y}=\frac{1}{i \omega \mu_{0}} \frac{\partial E_{x}}{\partial z},(19 \mathrm{a}) \\
H_{z}=-\frac{1}{i \omega \mu_{0}} \frac{\partial E_{x}}{\partial y},(19 \mathrm{~b}) \\
E_{y}=\frac{\sigma_{y z}}{D} \frac{\partial H_{x}}{\partial y}+\frac{\sigma_{z z}}{D} \frac{\partial H_{x}}{\partial z}+B E_{x},(19 \mathrm{c}) \\
E_{z}=-\frac{\sigma_{y y}}{D} \frac{\partial H_{x}}{\partial y}-\frac{\sigma_{y z}}{D} \frac{\partial H_{x}}{\partial z}+A E_{x},(19 \mathrm{~d})
\end{gathered}
$$

and the transfer functions determined. In general, the impedance tensor is nondiagonalizable, imitating locally a 3D subsurface, and the tipper vector is not null.

In order to understand more clearly the differences between the responses from 2D isotropic and anisotropic structures, I will focus on the simplest cases of decoupled equations, by comparing the solutions from the isotropic case to those from a $2 \mathrm{D}$ case with vertical anisotropy. For a given frequency $\omega$ the impedances and induction vectors at the surface have the following expressions:

A) 2D isotropic medium, with the strike along the $\mathrm{x}$ axis:

$$
\begin{gathered}
\underline{Z}_{2 D-i s o}=\left[\begin{array}{cc}
0 & Z_{x y}=f(\sigma(y, z)) \\
Z_{y x}=g(\sigma(y, z)) & 0
\end{array}\right], \\
\underline{\vec{T}}_{2 D-i s o}=\left(0, T_{y}=h(\sigma(y, z)) \cdot(20 \mathrm{~b})\right.
\end{gathered}
$$


B) 2D anisotropic medium, with vertical anisotropy along the measuring axes: (i.e. all the anisotropic bodies embedded in this 2D structure must have this same type of anisotropy):

$$
\begin{gathered}
\underline{Z}_{2 D-a n i s}=\left[\begin{array}{cc}
0 & Z_{x y}=f\left(\sigma_{x x}(y, z)\right) \\
Z_{y x}=g\left(\sigma_{y y}(y, z), \sigma_{z z}(y, z)\right) & 0
\end{array}\right],(21 \mathrm{a}) \\
\underline{\vec{T}}_{2 D-a n i s}=\left(0, T_{y}=h\left(\sigma_{x x}(y, z)\right) \cdot(21 \mathrm{~b})\right.
\end{gathered}
$$

Hence, the forms of the impedance tensor and the tipper are the same as in the 2D case. However, whereas in the isotropic case, both $Z_{x y}, Z_{y x}$ and $T_{y}$ depend on the distribution of $\sigma(y, z)$, in the anisotropic case $\sigma_{x x}(y, z)$ determines the values of $Z_{x y}$ and $T_{y}$ (TE mode), whereas $Z_{y x}$ (TM mode) depends on the distribution of both $\sigma_{y y}$ and $\sigma_{z z}$ along the y and $\mathrm{z}$ axes. The different sensitivities of TE and TM modes to the components of the conductivity tensor were already discussed in Saraf et al. (1986) and Osella and Martinelli (1993). This is also illustrated in an example of an anisotropic block shown in Jones (2006), where the TE mode fields (depending on $\sigma_{x x}$, which in the example has the highest conductivity value) are more attenuated than the TM mode (depending on $\sigma_{y y}$ and $\sigma_{z z}$, with lower conductivity values). As in the $1 \mathrm{D}$ case, a frequency to depth conversion has to be performed with care, as each mode investigates different depths for the same frequency. As for the tipper vector, it is important to note that, apart from depending on the variations of the conductivity across lateral boundaries, it is specifically the conductivity component along the strike $\left(\sigma_{x x}\right)$ that is responsible for the vertical magnetic field.

The fact that the TM mode is affected by the value of $\sigma_{z z}$ (as seen in Eq. 21a and as opposed to 1D anisotropic media, in which the responses are insensitive to it) was illustrated in the following example from Pek et al. (2008). It consists of three synthetic 2D models with an anisotropic body (vertical anisotropy) in each: a horizontal block, a vertical block and a horizontal layer on top of an isotropic block, and the corresponding responses, computed for different values of $\sigma_{z z}$ (Fig. $3)$. It can be seen how the responses are most sensitive to the value of $\sigma_{z z}$ for the 
third model, with the anisotropic layer on top of an isotropic block. The authors also emphasize the fact, arising from the first term of Eq. (16b) with derivatives on y, that $\sigma_{z z}$ is sensed mainly in the close vicinity of lateral conductivity gradients (in the third example, the lateral gradients would be between the isotropic block and the background).

For the case of dipping anisotropy the expressions of the impedance and the tipper would be similar to those in Eq. 21, although the TM mode component and the tipper would depend on the non-diagonal components of the conductivity tensor as well $\left(\sigma_{y z}(y, z)=\sigma_{z y}(y, z)\right)$ (see Eq. 17b). This dependence is illustrated in the example of $\mathrm{Li}$ (2002) (Fig. 4), where the responses of a model with an anisotropic block are computed for different values of the dipping angle.

Hence, the particularity of the responses of 2D models with vertical or dipping anisotropy (i.e. equations can be decoupled in 2 modes) is that the impedance tensor looks like those of an isotropic 2D Earth that varies with position and period. However, the tipper is only a function of the variations in $\sigma_{x x}$, so it is possible to find "apparent" inconsistencies between $Z_{x y}$ and $Z_{y x}$, and between $Z_{y x}$ and the tipper, $T_{y}$.

Figure 5 shows an example with three models, all with the same geometry (layer, 2 half-layers, half-space), and the corresponding responses, computed using the code of Pek and Verner (1997), for 40 sites, in the period range between $5 \mathrm{~s}$ and $5000 \mathrm{~s}$. The models are (A) a 2D isotropic model; (B) an anisotropic 2D model with the 2 half-layers vertically anisotropic, among which the value of $\rho_{x x}$ remains constant whereas the values of $\rho_{y y}$ and $\rho_{z z}$ are different; and (C) a 2D anisotropic model, also with vertically anisotropic half-layers, but with different values of $\rho_{x x}$ and the same values of $\rho_{y y}$ and $\rho_{z z}$. The responses for the isotropic model show vertical and lateral changes in resistivities and phases in both the TE and TM modes, and due to the lateral discontinuity (between $1 \Omega \cdot \mathrm{m}$ and $100 \Omega \cdot \mathrm{m}$ ), the real induction arrows (in red) at the center of the model point towards the conductive side. For the first anisotropic model (B), the responses in the TE mode are the same as in a homogeneous half-space of $10 \Omega \cdot \mathrm{m}$ (no change in conductivity in the "xz" plane), whereas the TM mode responses are similar to the ones in the 
isotropic model, reflecting the changes between the isotropic $10 \Omega \cdot \mathrm{m}$ (top and bottom layers of the model), and the anisotropic values $\rho_{y y}(1 \Omega \cdot \mathrm{m}$ and $100 \Omega \cdot \mathrm{m})$ and $\rho_{z z}(100 \Omega \cdot \mathrm{m}$ and $1000 \Omega \cdot \mathrm{m})$ between the two half-spaces. Induction arrows are null (in accordance with TE horizontal responses, but apparently inconsistent with the TM ones). For model $\mathrm{C}$, the contrary effect is observed; TE mode data exhibit lateral changes due to the variation of $\rho_{x x}$ and induction arrows pointing to the conductive side, as one would expect. The TM mode responses resemble those of a stratified medium (because there is no lateral change in $\rho_{y y}$ and $\rho_{z z}$ ).

For the general 2D anisotropic case (when Maxwell equations cannot be decoupled), it is not easy to describe the responses from an analytical point of view. However, from studies using synthetic and real data, it has been possible to describe features that characterize the responses from 2D anisotropic media, keeping in mind that, in general they resemble those of a 3D isotropic media.

Pek (2009) presents a comprehensive summary of the effects of anisotropic conductors on MT data, emphasizing several works done on the effects of 2D models with arbitrary anisotropy, including the analyses described in Pek and Verner (1997).

These effects can be classified as:

(1) Deflection of the induction arrows

In a 2D isotropic Earth, real induction arrows are considered an undistorted strike indicator, being perpendicular to it, and pointing away from (or towards, using Parkinson (1962) convention) conductive areas. On the contrary, anisotropic structures attract the currents induced in the Earth towards the direction of the preferred conductivity in the anisotropic domains, and the induction arrows can be deflected, to lower or higher degrees, depending on the depth and extent of the anisotropic domains. This implies that the strike directions indicated by the impedance tensor and the induction arrows are inconsistent. Figure 6 shows two examples from Pek and Verner (1997), with a model for which this deflection is towards the direction perpendicular to the anisotropic strike (model A), which is what would be expected intuitively; and a second model (model B), for which the 
effect of the anisotropy is that the induction arrows become almost parallel to the anisotropic strike as a consequence of a chain of induction processes under the inter-mode coupling conditions. Weidelt (1999) also uses an example of a 2D model with an anisotropic conductor (with principal directions not aligned to the measuring directions) overlaying an isotropic layer with lateral varying conductivity, showing how the vertical field is generated and deflected due to the anisotropic conductor.

In a real context, Brasse et al. (2009) presented, for the South-Central Chilean continental margin, a 2D model with a lower crust formed by different anisotropic domains (Fig. 7A), where the responses fit the measured induction arrows (pointing NE), which was not possible using a 3D model. In the proposed model, the ocean (East) extends below the continental NE oriented anisotropic layer and both structures are coupled. Thus, the resulting induction arrows (P4) are deflected from what would result from a simple vectorial addition (P3) of the ocean (P1) and the anisotropic layer (P2) induction arrows.

(2) Distortion effects in the responses

The responses of 2D models with generally anisotropic bodies can be highly distorted, in the sense that the diagonal components of the impedances dominate over the non-diagonal ones, and that the phases of the principal impedances roll out of their natural quadrant (Phases Rolling out of Quadrant, PROQ, as described by Chouteau and Tournerie 2000). These effects have been simulated by an outcropping anisotropic block, with the anisotropic azimuth different than the structural azimuth, either within an isotropic background (Pek 2009) or underlain by an anisotropic layer with principal directions orthogonal to the ones of the block (Heise and Pous 2003).

The PROQ effect is a consequence of the continuity of the normal current densities and tangential electric field across a contact separating two media of different conductivities. In a 2D model with the strike along the x direction, any discontinuity would be along the $\mathrm{x}$ direction too, and this condition applies for the electric field in the TE mode $\left(E_{x}\right)$, which is always continuous, and for the current density in the y direction (assuming that the discontinuity is vertical) $\left(j_{y}=(\sigma \cdot \boldsymbol{E})_{\mathrm{y}}\right)$. 
If the media on each side of the discontinuity are isotropic with $\sigma_{1}$ and $\sigma_{2}$, this implies that $E_{x 1}=E_{x 2}$ and because $j_{y}=\sigma \cdot E_{y}$, then $\sigma_{2} E_{y 2}=\sigma_{1} E_{y 1}$; hence $E_{y 2}=\left(\sigma_{1} / \sigma_{2}\right) E_{y 1}$. Because $\sigma_{1} / \sigma_{2}$ is a real number, the amplitude of $E_{y}$ changes but not its phase. If the media are anisotropic, the conductivities are tensorial and the condition of the current densities implies that $E_{x l}=E_{x 2}$ and $\left(\sigma_{2} \boldsymbol{E}_{2}\right)_{y}=\left(\sigma_{1} \boldsymbol{E}_{1}\right)_{y}$. In this case, unless the anisotropy is aligned with the measuring axes or for a specific combination of the tensor and field components, the phases of $E_{y 2}$ and $E_{y 1}$ will be different, and it is possible that one of these moves from its natural quadrant.

Pek (2009) shows an example of an outcropping anisotropic block, within an isotropic layered host, with an anisotropic strike of $30^{\circ}$ (Fig. 8). The responses of the model show both effects described, phases rolling out of quadrant, and significantly high diagonal components. Specifically, PROQ is identified when the measuring directions are away from both the structural and the anisotropy strike.

Heise and Pous (2003) studied in detail the responses generated by a 2D model with an anisotropic block underlain by an anisotropic layer (modified from Pek and Verner 1997), having different anisotropic strikes (Fig. 9), that were characterized by phases out of quadrant at some sites (on top of the block or close to its edges) and periods. By varying different parameters of the model, they concluded that the phases out of quadrant happens when the anisotropy radio is high (either in the block or in the layer), the host medium is more resistive than the maximum resistivity of the anisotropic block, the difference between anisotropic strikes is high, and the block is near the surface and relatively narrow. The authors show, in terms of the electric field, that PROQ occur for the TM mode impedances. It is also important to note that these effects mainly affect the sites near the anisotropic block, leaving the rest of the 2D regional structure unaffected. From this study the authors generated a 2D anisotropic model with shallow anisotropic anomalies to fit the MT data measured in the Ossa Morena Zone (SW Iberia), where anomalous phases at some sites occurred. In contrast, the rest of sites, away from the anisotropic blocks, could be inverted using a 2D isotropic code. Similar tests were performed by Chen et al. (2009), with data from South Africa, leading to similar conclusions, and in Weckmann et al. (2012), who 
discard the data with phases out of quadrant in the 2D isotropic inversion, as those do not affect the general regional structure.

Overall, the responses of a generally anisotropic 2D medium are characterized by apparent inconsistencies between the strike direction (defined on the basis of the properties of the MT impedance tensor) and the tipper vectors, and by the phases rolling out of their natural quadrants. The first effect can be observed even when anisotropy is aligned with the 2D structure (e.g. Fig. 5, where there is a clear strike direction but the tipper can be null), whereas the phases out of quadrant are observed when the anisotropic bodies are not aligned with the structure, as a consequence of the boundary conditions on the margins of the anisotropic bodies. However, phases out of quadrant can be also explained by the data being affected by galvanic distortion (Chouteau and Tournerie 2000, Lilley and Weaver 2010), 2D structures with high resistivity contrasts (Selway et al. 2012) or certain 3D conductive bodies generating strong current channelling (Lezaeta and Haak 2003), such as an L-shaped body (Ichihara and Mogi 2009). An example with a superposition of these effects was given by Weckmann et al. (2003) in which the complexity of the data is explained by a model with three main features: a shallow conductive ring structure, a crustal anisotropic layer and an elongated conductor producing current channelling. A procedure that is often used to rule out 3D effects is to create a 3D model and calculate the responses. If, after testing the grid resolution, the $3 \mathrm{D}$ model responses cannot fit both the impedances and the induction arrows, then the data must be anisotropic (e.g. Padilha et al. 2006, Brasse et al. 2009, Ruiz-Constán et al. 2010). However care must be taken in this approach as, in order to justify anisotropy, all the responses should fail to fit the 3D model, unless there are other geological or geophysical evidences.

\section{3) 3D anisotropic models}

In a 3D anisotropic Earth, the general equations (3a) and (3b) have to be developed without any simplification and the solutions have a general form, with generally non-diagonalizable impedances, possibly phases out of quadrant, and no specific patterns to the induction vectors. As already mentioned in section 2.2, responses from an anisotropic model should also be interpretable by an isotropic 
model of arbitrary complexity (Weidelt 1999). According to Weidelt, this is a consequence of the EM induction studies imaging spatial averages, for which the microstructure of the conduction mechanisms is inaccessible, and, hence, all anisotropy can be explained by structural anisotropy resulting from spatial averages over isotropic structures with a preferred orientation. However, this equivalence should be understood as a practical problem resulting from the limited resolution of the MT method, and not as a rigorous statement (J. Pek, pers. comm.).

\section{4) Anisotropy, galvanic distortion and impedance tensor decomposition}

To finish this section, I will comment on the galvanic effects generated by smallscale superficial bodies on the responses of models with anisotropy. In MT, galvanic distortion can be modeled as a $2 \times 2$ real matrix that multiplies the impedance tensor. In isotropic situations, it is possible to identify and remove (sometimes with an unknown and unknowable scale effect) galvanic effects over $1 \mathrm{D}$ and $2 \mathrm{D}$ structures. In three-dimensional geoelectric structures it is not easy to perform the decomposition unless the characteristics of the distortion are well known, although several approaches have been proposed (e.g. Jones $2012 \mathrm{~b}$ and references therein).

For 1D anisotropic structures, some theoretical studies have been performed on the effects of galvanic distortion:

Santos and Mendes-Victor (2000) compare impedance responses affected by galvanic distortion for 2D isotropic structures (3D/2D) and two particular cases of $1 \mathrm{D}$ anisotropic structures $\left(3 \mathrm{D} / 1 \mathrm{D}_{\text {anis }}\right)$. In the principal directions of a $2 \mathrm{D}$ structure affected by galvanic distortion, the impedance tensor becomes:

$$
\underline{Z}_{3 D / 2 D}=C \cdot Z_{2 D}=\left[\begin{array}{ll}
c_{1} & c_{2} \\
c_{3} & c_{4}
\end{array}\right]\left[\begin{array}{cc}
0 & Z_{x y} \\
Z_{y x} & 0
\end{array}\right]=\left[\begin{array}{cc}
c_{2} Z_{y x} & c_{1} Z_{x y} \\
c_{4} Z_{y x} & c_{3} Z_{x y}
\end{array}\right] .
$$

In the axes of the anisotropy for a 1D model with a single anisotropic layer (1Danis1) (or different anisotropic layers with the same principal axes orientations), 
affected by galvanic distortion, the distorted impedance tensor takes the same form:

$$
\underline{Z}_{3 D / 1 D-a n i s 1}=C \cdot Z_{1 D-a n i s 1}=\left[\begin{array}{cc}
c_{1} & c_{2} \\
c_{3} & c_{4}
\end{array}\right]\left[\begin{array}{cc}
0 & Z_{x y} \\
Z_{y x} & 0
\end{array}\right]=\left[\begin{array}{ll}
c_{2} Z_{y x} & c_{1} Z_{x y} \\
c_{4} Z_{y x} & c_{3} Z_{x y}
\end{array}\right],
$$

whereas if the 1D model has more than one anisotropic layer, with different principal axes (1D-anis2), then the distorted impedance tensor becomes:

$$
\underline{Z}_{3 D / 1 D-a n i s 1}=C \cdot Z_{1 D-a n i s 2}=\left[\begin{array}{cc}
c_{1} & c_{2} \\
c_{3} & c_{4}
\end{array}\right]\left[\begin{array}{cc}
Z_{x x} & Z_{x y} \\
Z_{y x} & -Z_{x x}
\end{array}\right]=\left[\begin{array}{cc}
c_{1} Z_{x x}+c_{2} Z_{y x} & c_{1} Z_{x y}-c_{2} Z_{x x} \\
c_{4} Z_{y x}+c_{3} Z_{x x} & -c_{4} Z_{x x}+c_{3} Z_{x y}
\end{array}\right],
$$

where the reference frame can be aligned with one of the layers' principal axes. After performing a 3D/2D decomposition to recover the regional responses using synthetic data, the authors indicate the difficulty of recovering the undistorted tensor in the second anisotropic case.

Kováčiková and Pek (2002), as an application of the Ricatti equations developed for 1D anisotropic media, studied the influence of a depth-variable regional strike on magnetotelluric decomposition results, with the variable strike simulated by a variable anisotropy within the $1 \mathrm{D}$ model. When the anisotropic layers are separated, the inclusion of magnetic distortions allows for a better retrieval of the strike directions.

Jones (2012a) tackled the problem of removing galvanic distortion in 1D anisotropic responses (for a general model, as in Eq. 24), following a hypothesis testing statistical approach with a parametrization used for 3D structures, 3D/1Danis. The following example was used to test the approach. A synthetic 1D model of the lithosphere and asthenosphere with 2 anisotropic layers was created (used to describe the MT responses over the Great Slave Lake shear zone in northwestern Canada), and the responses were computed using the 1D code of Pek and Santos (2002) (Fig. 10A). Noise and galvanic distortion (twist angle of $5^{\circ}$ and shear angle of $+30^{\circ}$ ) were added to the responses (Fig. 10B). Using a 
$3 \mathrm{D} / 2 \mathrm{D}$ approach, the resulting distortion parameters are inconsistent because the strike angle does not match with any of the anisotropic layer azimuths, and neither the twist nor the shear values are properly recovered. If the new approach $3 \mathrm{D} / 1 \mathrm{Danis}$ is applied, the distortion values are properly recovered and the corrected responses are consistent with the original undistorted ones (Fig. 10C). The method was also applied to data from Central Germany (DIE site) and the Great Slave Craton shear zone.

The formulation of the galvanic distortion decomposition for more complex anisotropic structures $\left(3 \mathrm{D} / 2 \mathrm{D}_{\text {anis }}\right.$ or $\left.3 \mathrm{D} / 3 \mathrm{D}_{\text {anis }}\right)$ remains unsolved, given the inherent inability of the method to separate structure and anisotropy from distortion effects.

\subsection{Dimensionality tools}

In an isotropic Earth, dimensionality analysis allows discerning if the regional geoelectrical structures are 1D, 2D or 3D and whether the MT responses are affected or not by galvanic distortion. Dimensionality analysis methods are commonly based on the use of the invariant parameters of the impedance tensor and a statistical analysis of the data errors (see Martí 2006, Chapter 2 and references therein). The same procedure can be extended to anisotropic structures, but, due to the higher number of parameters involved, additional information is needed, such as the induction vectors, or the joint behaviour of different sites and periods.

This complementarity between the use of rotational invariants and the comparison between responses at different sites was already highlighted by Reddy and Rankin (1975) some 37 years ago. They compared the responses from 3D isotropic structures and 2D anisotropic ones (with general anisotropy). In both cases, the "additional impedances" $\left(Z_{x x}\right.$ and $\left.Z_{y y}\right)$ are not necessarily zero, and both the skew $\left(\left(Z_{x x}+Z_{y y}\right) /\left(Z_{x y}-Z_{y x}\right)\right)$ and ellipticity $\left(\left(Z_{x x}-Z_{y y}\right) /\left(Z_{x y}+Z_{y x}\right)\right)$ do not vanish. However, in the $3 \mathrm{D}$ cases, the dimensionality coefficients and the impedances are space dependent, varying from one sounding to another or from one profile to another; whereas in anisotropic 2D structures, these parameters are invariant for profiles 
perpendicular to the structural strike. A very important consequence of this is that it is always possible to use an array of magnetotelluric soundings to distinguish anisotropic, two-dimensional structure from isotropic, two-dimensional structures or three-dimensional structures.

Nowadays, the most common methods used for dimensionality analysis are the Strike Decomposition Code (McNeice and Jones 2001), the Phase Tensor (Caldwell et al. 2004) and the WAL invariants criteria (Weaver et al. 2000). The Strike Decomposition Code is a hypothesis testing method to assess how well the data fits a 3D/2D model and thereby extracts the best fitting $1 \mathrm{D}$ or $2 \mathrm{D}$ impedances statistically consistent with the full dataset. The phase tensor has the advantage that it is invariant under distortion, so it provides information on the regional structures, whereas the WAL invariant method extracts full information from the impedances and allows identifying distortion and recovering the regional strike if applicable. The three methods are well established for isotropic structures. It has only been more recently that these methods have been tested to analyse responses from anisotropic media.

Regarding the use of the Strike Decomposition Code, it has been observed that for 2D anisotropic models, the strike directions computed at a single site change along with the period (Heise and Pous 2003), and how the strike direction and distortion parameters can be incorrectly determined, except in areas where the twist and shear parameters are close to zero (Miensopust and Jones 2011).

Heise et al. (2006) analysed the relationship between anisotropy and phase splits using the phase tensor along with the resistivity and phase responses computed from synthetic anisotropic models. For an anisotropic half-space, no phases splits are produced and the phase tensor ellipses reduce to circles of unit one. For 1D models with a layer with vertical and dipping uniaxial anisotropy, the phase split is caused by the conductivity change at the interface between layers, and the phase tensor ellipses are oriented along the horizontal and vertical axes (Fig. 11). The responses would be the same as in a $2 \mathrm{D}$ isotropic model only that in the anisotropic cases at each site the same phase tensor sequence would be observed. If the anisotropy of the layer has a different orientation, the phase tensor principal 
axes are determined by the orientation of the equivalent azimuthal anisotropy. In these examples, the phase tensors themselves do not provide enough information to identify anisotropy, and hence, in general, phase splits should not be interpreted as being solely caused by anisotropic structures. In $2 \mathrm{D}$, an example is shown to illustrate the difficulty to distinguish between anisotropic and isotropic models, which produce very similar phase tensor ellipses and induction arrows (Fig. 12). The main discussion of the paper focuses on the fact that MT phase splits are different from seismic shear wave splits, which are caused by the bulk anisotropic properties of the material, as evidenced by the fact that a shear wave split will be observed at the surface of an anisotropic body but an MT phase split will not. Direct information on the bulk conductivity is contained only in the MT amplitude response, although it can be subject to distortions, making its interpretation difficult. Preliminary results from the study from Rödder and Junge (2012) shows how, by combining the information from the MT phase tensor and the DC apparent resistivity tensor, it is possible to identify uniquely the presence of anisotropy.

Martí et al. (2010) studied the imprints of anisotropic media responses in the WAL rotational invariants. The tests were made using synthetic $1 \mathrm{D}$ and $2 \mathrm{D}$ anisotropic models, computing the responses with the code of Pek and Verner (1997), and adding 1\% random noise. The dimensionality analysis was then performed using the WALDIM (Martí et al. 2009) code, which takes into account the data errors. The results allowed extending the WAL dimensionality criteria to include extra conditions that allow anisotropic media to be distinguished from 2D isotropic ones (Table 2). An exception is the case where the principal anisotropy directions are aligned with the measuring axes, then the information from the induction arrows might be necessary (as noted in the synthetic tests of Berdichevsky and Pushkarev 2006). Also, except in simple cases, anisotropy cannot be identified from one site alone, and it is necessary to check for the full dimensionality pattern at different periods (or sites), as in the following example.

Figure 13 shows two models with 2 anisotropic layers each, with different anisotropic strike. The dimensionality pattern for both is, from the shortest to the longest period, 1D (corresponding to the first isotropic layer), $2 \mathrm{D}$ with $30^{\circ}$ strike 
direction (corresponding to the first anisotropic layer), 3D (due to an abrupt increase in the value of invariant $I_{7}$, caused by the inclusion of the second anisotropic layer), and finally $2 \mathrm{D}$, with approximately $39^{\circ}$ strike, a value in between the two anisotropic strike values of the two layers, $30^{\circ}$ and $45^{\circ}$. In all $2 \mathrm{D}$ cases, after a statistical analysis of the results, the directions $\theta_{2 \mathrm{D}}$ (computed independently from the real and imaginary part of the MT tensor) and $\theta_{3 \mathrm{D} / 2 \mathrm{D}}$ (computed combining both real and imaginary parts) are non-significantly discrepant.

WAL dimensionality analysis was also carried out for the 2D models in Heise et al. (2006) (models A and B, Fig. 12). The dimensionality pattern for the anisotropic and isotropic models (models $4 \mathrm{a}$ and $4 \mathrm{~b}$ in Fig. 14) is almost the same. A third model was created as a modification of model 4a by rotating the anisotropic layer by $30^{\circ}$. The dimensionality results (model 4c in Fig. 14) show a more complex pattern, with 2D cases with different values of the strike computed from the real or imaginary part of the tensor, which is an indication of anisotropy.

The comparison of the dimensionality description obtained using the WAL invariants criteria with the phase tensor diagrams in the tests performed, allowed us to conclude that in some cases both provide the same information (although in the paper Martí et al. (2010) there is an error in the representation of the phase tensor ellipses in the example of the 2D anisotropic model). Nevertheless, if phases do not change with the period, such as in the case of an anisotropic halfspace, only WAL criteria allow anisotropy to be identified.

\section{Inversion of anisotropic data}

An appropriate inversion process in MT is a crucial step in order to obtain a reliable model that fits the measured data and is consistent with other observed data. The problems that might arise when not using the appropriate approaches according to the dimensionality of the data are well known in isotropic contexts (Ledo 2006 and references therein). Added to the fact of the general nonuniqueness of the solutions to the inverse problem, in anisotropic situations additional conductivity components have to be resolved, giving rise to a broader 
range of possible solutions. Some studies that compare the effects of inverting anisotropic data using isotropic codes have been carried out, which have been compared with the first results from new anisotropic inversion codes.

The effects of inverting anisotropic data using an isotropic 2D code were studied by Heise and Pous (2001), before anisotropic inversion codes became available. The authors showed that it is possible to obtain a macro-anisotropic model comprised of a sequence of conductive and resistive dykes jointly inverting TE and TM modes for some anisotropic parameters and by using the correct strike direction. Wannamaker (2005) suggests an alternative procedure, consisting in inverting one mode or combined modes for the period range where the strike angle is constant. Then to invert the other mode's data, keeping the model close to the previous one, to obtain a smoother estimate of the deep anisotropic conductivity values.

Since these studies, great progress has been made with regard to the development of anisotropic inversion codes, and consequently a better understanding of the effects of anisotropy in either isotropic or anisotropic inversion. In the following, I will change the discourse to some of the most recently appeared MT inversion code and their applications, along with new studies on the effects of inverting anisotropic data using different approaches.

Pek and Santos (2006) presented their 1D azimuthally anisotropic algorithm, which can be used as a practical tool to understand and analyse anisotropic data in a simple context and to design 2D (or even 3D) test models for anisotropy simulation studies. The inversion procedure allows for different regularization parameters, such as smoothness and anisotropy ratio. The code was used with a subset of MT data from the South Portuguese Zone and Ossa Morena Zone in southern Portugal, with geological and geophysical indications on possible anisotropy due to shearing and graphitization. The resulting 1D anisotropic models were then used to generate an initial 2D anisotropic model, which was modified by trial and error. 
As a useful tool for constrained inversion, Roux et al. (2011) put forth a genetic algorithm code to jointly invert long period MT data and Rayleigh surface-wave dispersion curves, for 1D azimuthally anisotropic media, based on the isotropic Genetic Algorithm scheme of Moorkamp et al. (2010). In their approach, the connection between the electrical and seismic models is in their coincident interfaces and, within each layer, free parameters are the principal resistivities, shear-wave velocities, the corresponding azimuths (which in some cases, such as in the asthenosphere, are required to be the same), and thickness. The authors applied the algorithm to invert MT and surface-wave datasets from Central Germany (DIE site), which validated the code and provided new information on the deep structure of the region. The model obtained (Fig. 15) allowed delineation of a common electrical and seismic LAB (Lithosphere-Asthenosphere Boundary) at approximately $84 \mathrm{~km}$. It also showed an emerging agreement between the two anisotropic coefficients, of around one order of magnitude (less than the two orders of magnitude defined on the basis of MT data alone by Gatzemeier and Moorkamp 2005), with the electrical anisotropy most likely explained by hydrogen diffusion (Gatzemeier and Tommasi 2006).

Using the same data, but after removing galvanic distortion effects (Jones 2012a), Mandolesi and Jones (2012a) presented a joint inversion scheme based on mutual information. In this scheme a reference model is fixed and the information shared with the conductivity structure is maximized, through the computation of the joint probability distribution and the minimization of the entropy. As a reference model, the authors used a seismic model with minimum RMS, and the MT data corrected for distortion. The results agree with previous studies and the strength of the method is that the use of an independent dataset as reference improves the resolution in the resistive direction in the asthenosphere.

The first application of a 2D anisotropic inversion code was presented by Baba et al. (2006) in marine MT data from the East Pacific Rise within the MELT experiment, using the code of Mackie (2002) (which also corrects 3D topographic effects in the data). This code searches for a 2D anisotropic model with anisotropy aligned with the structural directions (along and across strike, and vertical), that fits either the TE and TM resistivity and phase or the vertical magnetic transfer 
function, or all three. It uses the method of nonlinear conjugate gradients (NLCG) with two regularization parameters: smoothness and anisotropy. In this case, strike was along the rise axis, and TE and TM resistivities and phases were inverted.

Given the broad range of possible models that such types of inversion can generate, different values for the smoothness and the anisotropy ratio regularization parameters as well as initial models were tested. The final anisotropic model presented a better data fit than previous isotropic models (as expected due to the greater number of degrees of freedom permitted). An isotropic resistive zone was interpreted as a region of mantle that has undergone melting at the ridge and has been depleted of water, whereas vertical and horizontal anisotropic features were related with melting aligned in the vertical direction and to the presence of hydrogen in olivine (Fig. 16). The code was also used in the studies of Matsuno et al. (2010) for the Mariana subduction system, where anisotropy was found to be weak; and Evans et al. (2011) in the Kaapvaal craton, which model was compatible with an isotropic model as well.

Pek et al. (2011) presented the state of the art of their 2D inversion code for arbitrary anisotropy, which, although still in a raw stage, shows promising results, as illustrated in application to real data. The inversion is based on Occam's inverse strategy, using NLCG, where the penalties are the structural complexity and anisotropy. The forward problem and parametric sensitivities can be computed using finite volumes. Model parameters are the 6 conductivity components at each cell and the inverted data are the 4 complex components of the impedance tensor plus the 2 complex vertical transfer functions, for a total of 12 data per frequency. Fig. 17 shows an example of an inversion from synthetic data. The true model (bottom right panel) consists of a near-surface anisotropic block, underlain by a layer separated in sections with different anisotropic properties. This type of model can produce MT phases rolling out of their natural quadrant, which may be a source of difficulty for the inversion procedure. However, in this particular case, it does not happen in the model coordinate frame. The inversion was run for all three principal resistivities and for the anisotropic strike, assuming uniaxial anisotropy. In general, the inverse model captures the true structure satisfactorily, especially the anisotropic block and the right most anisotropic layer. The authors conclude that, as in the 1D case, the most reliable 
inversions result from structures with azimuthal anisotropy and the anisotropic dip is unresolvable without additional information; vertical resistivity is almost indistinguishable in 2D models in spite of its irreplaceable role in the forward responses; and, because of the structural and anisotropy penalties, care must be taken when interpreting resistivities from dipping structures. In application to real data, the code was used to invert data from the Southern Portuguese Zone and the Ossa Morena Zone in southern Portugal (Pous et al. 2004), and from the BC87 data set, obtaining a satisfactory fit to the experimental data. However, a lot of open questions still remain, such as how do static distortions and 3D effects interfere with 2D anisotropic structures, how one determines the structural strike, and issues related to the inversion of anomalous MT phases.

As already said, there are no 3D anisotropic inversion codes available in MT. However, a starting point can be the 3D inversion codes for marine CSEM data, with transverse anisotropy. Newman et al. (2010) successfully applied his 3D code with transverse anisotropy in synthetic and real (Troll field and Campos Basin) contexts. The code solves the forward problem using finite differences and the inversion is performed using non-linear conjugate gradients based on a regularized least squares approach. The authors have extended the code to jointly image CSEM and MT data, to improve the resolution, although this has not been applied yet (G. Newman, pers. comm.), and they also point at the difficulty of considering arbitrary anisotropy. Sasaki (2011) presented an anisotropic 3D joint inversion code for CSEM and MT data, with transverse anisotropy, based on the Gauss-Newton approach and finite difference modeling. Using a synthetic example, the author emphasizes the fact that, in the presence of an anisotropic reservoir, CSEM inline data has sensitivity on the vertical resistivity and the overburden, whereas broadside data is more sensitive to the horizontal resistivity. Hence, MT (mainly sensitive to horizontal resistivity) can improve the resolution of the model, especially when only inline data are available.

Miensopust and Jones (2011), motivated by the presence of an unusual bent conductive body in a 2D model obtained in the ZIM profile (northeastern Botswana) using an isotropic code, studied the artefacts that can be obtained when inverting anisotropic data for isotropic models. They used different 2D models 
with a layered isotropic background and anisotropic blocks, resembling the resistivity distribution found in the study area, where anisotropy is due to the presence of a dyke swarm. The responses were computed using the code of Pek and Verner (1997) and inverted using the code of Mackie (2002), by enforcing isotropic models and anisotropic ones for comparison, and by changing different parameters in the model. For isotropic inversions, the effects created by the anisotropic structures are that resistivity values can be overestimated, horizontal conductive layers can appear downward bent, and interface depths might not be properly located. Such artefacts are more evident for large anisotropic strikes and the wider and thicker are the anisotropic blocks. The results from these tests made suspect that the conductive area observed in the ZIM profile is probably an artefact due to anisotropy. This conductor is located under the Okavango Dyke Swarm (ODS), where dykes have an azimuth of $75^{\circ}$ with respect to the profile azimuth. By inverting the data from this area using the anisotropic code, the ODS extends only to crustal depths, and the conductor beneath appears more horizontal (Fig. 18).

Another study was performed by Schmoldt and Jones (2012), which presented an approach to invert 3D data with two oblique directions, using 1D anisotropic (Pek and Santos 2006) and 2D anisotropic (Mackie 2002) inversions. The tests were performed by inverting data from a 3D model with two orthogonal crust and mantle structures, along a profile oblique to both structures. The 2D models obtained (or stack of 1D models) from the inversion results allowed the identification of certain features of the 3D model.

\section{Case studies}

Finally, in this section I would like to mention some of the case studies involving anisotropy using MT that have appeared since the last review by Wannamaker (2005).

Frederiksen et al. (2006) combined MT, seismic (Lithoprobe) and teleseismic (from the POLARIS experiment) data to study upper-mantle fabrics of the Eastern 
Canadian Shield across the Grenville Front. The geoelectrical strike agrees well with seismic SKS results, for which they conclude that the upper lithosphere contains thin anisotropic layers, related to relicts of lower crustal materials or slabs related to paleo-subduction; whereas the lower lithosphere is more ductile and uniformly anisotropic.

Rosell (2012) revisited the MT data from the Alberta Province, where previous studies (Boerner et al. 2000) had shown solid evidence of anisotropy at lithospheric levels, and added data from a new profile to the study. The dimensionality analysis of the data using complementary tools (WALDIM and Strike decomposition) led to contradictory results, which was interpreted as being caused by the presence of electrical anisotropy. The results from the models constructed using a 2D anisotropic code allowed identifying anisotropy in both the crust and lithospheric mantle.

In the MT study of Wannamaker et al. (2008) across the Great Basin - Colorado Transition Zone, the 2D model obtained from isotropic inversion imaged two narrow steep conductors separated by steep resistive zones, which were identified as artefacts due to the presence of anisotropy. This anisotropy was interpreted as interconnected melt in the upper mantle, which has supplied magma to the lower crust, consistent with shear wave splitting observation and extensional mantle melting models.

Naif et al. (2013) inverted MT data from the subduction zone of the Cocos plate offshore Nicaragua using a 2D code (Key and Ovall 2011) including anisotropy. The resulting model images, beneath a resistive oceanic lithosphere, an anisotropic conductive layer ( $45-70 \mathrm{~km}$ depth), which is interpreted as a partially molten layer, with higher conductivity in the direction parallel to the plate motion. This indicates that the melt has been sheared into tube-like structures, and that the LAB acts as a low viscosity channel that decouples the overlying brittle lithosphere from the deeper convecting mantle.

Brasse et al. (2009) characterised the lower crust in the South Chilean continental margin as anisotropic, interpreted as caused by the presence of fluids and 
fractures, with a direction oblique to the continental margin but in accordance with the stress field in the region of the volcanic arc.

Padilha et al. (2006) analysed the geoelectric strikes from MT data and shear wave splitting parameters to study the degree and orientation of strain in the subcontinental mantle in central-southeastern Brasil. 3D forward modelling tests indicated that the ocean affects the tipper magnitude and direction, whereas an anisotropic layer in the upper mantle accounted for the measured strike directions, which agree with the fast polarization of S-waves. The anisotropy ratio is low, so it can be interpreted as intrinsic anisotropy of aligned olivine crystals, with some additional mechanism in localized regions.

In the Betic Cordillera (Western Mediterranean) Ruiz-Constán et al. (2010) inferred the presence of electrical anisotropy in the upper mantle based on analysis of the strikes and induction arrows from an MT NS profile. However, the study of Martí et al. (2012) of a broader area, analyzing data from both the impedances and the tippers, found that the data are influenced mainly by $3 \mathrm{D}$ effects.

In the African continent, several MT studies have shown evidence of electrical anisotropy (Weckmann 2012 and references therein). Here are the most recent:

Hamilton et al. (2006) inferred the presence of electrical anisotropy from the analysis of MT strikes in the southern African lithosphere, and compared the results with SKS data. Because of the broad range of penetration depths in the area, the Strike Decomposition was performed after a frequency to depth NiblettBostick (NB) conversion. In the crust, the directions of the anisotropy change across terrane boundaries. In the upper mantle the results are not consistent with the SKS results, for which electrical anisotropy is mainly caused by large-scale structures, and the causes for seismic anisotropy probably have a weak electrical signature. Jones et al. (2009b) presented maps of the electrical anisotropy at 100 $\mathrm{km}$ and $200 \mathrm{~km}$ for the same region, computed from an NB approximation, and correlated the limits between the isotropic and anisotropic as target areas for the possible presence of diamondiferous kimberlites. 
Häuserer and Junge (2011) studied the crustal structure of the Rwenzori Mountains in Uganda using an MT survey. Using an alternative representation of the phase tensor (in the form of bars) the disagreement between the phase tensor invariants and the small induction arrows was explained as anisotropy directly beneath the Moho, with an anisotropy ratio of at least 10, which might be caused by the presence of wet olivine in the upper mantle with a preferred orientation.

In the Eastern Indian Craton, Shalivahan and Bhattacharya (2005) performed an MT broadband study, where data were modeled as 2D, as indicated by dimensionality analysis results. The model showed a rare high resistive continental lower crust (1 S conductance), which provided a window to resolve the mantle structure underneath. 1D anisotropic modelling (suggested by the consistent phase splitting of the data and negligible induction arrows) depicted an anisotropic mantle at depths of $175 \mathrm{~km}$, with a direction oblique to the present-day Indian Plate movement. The discrepancy was interpreted as either resistance to mantle flow by present-day motion, complex convection of the mantle or a combination of both.

Patro et al. (2005) studied the crustal structure below the Deccan Flood Basalts (India). The isotropic models showed high resistive values with the presence of subvertical zones of enhanced conductivity in the middle-lower crust. These were interpreted as partly reactivated faults and fractures of the Precambrian basement, and the use of a forward anisotropic code confirmed the presence of conductive dykes in the lower crust.

Le Pape et al. (2012) revisited the magnetotelluric models from the northern part of the Tibetan Plateau and performed and anisotropic inversion (using the code of Mackie 2002), obtaining evidences of anisotropy, which was interpreted to be related to melt intrusion north of the Kunlun Fault. The geometry of this intrusion was tested in a 3D model, which responses agreed with a fingered-type intrusion, as opposed to a single intrusion. South West of this region, in the Main Central Thrust Zone of the Sikkim Himalaya, Pavan Kumar and Manglik (2012) observed anomalous MT phases which were modelled locally as an anisotropic block on top 
of an anisotropic layer with different anisotropic strikes. The model was supported by the presence of graphyite bearing schists within the High Himalayan Crystallines, although other hypotheses (such as strong resistivity contrasts) were not ruled out.

Heinson and White (2005) illustrate two possible interpretations for the electrical nature of the lithosphere of the North Australian Craton from a marine MT study in the Gulf of Carpentaria, off the north coast of Australia. Using 1D or 2D anisotropic inversions, the data can fit either a model with anisotropy (with an anisotropy ratio higher than 100) in the lower crust, or a 2D model with an isotropic lithosphere with lateral variations in upper mantle resistivity of a factor of 10. However, from other geological and geophysical constraints the heterogeneous model is preferred, where the variations in resistivity may be due to temperature changes.

\section{Summary and Conclusions}

This paper has reviewed the main theoretical aspects necessary to understand how electrical anisotropy in the Earth affects magnetotelluric responses in different dimensionality settings, including the main steps involved in the analysis, modelling and inversion of MT data.

To summarise, I will address again the questions arisen in the introduction:

a) How does anisotropy affect the magnetotellurics responses?

In 1D anisotropic models, the impedance responses resemble those from 2D isotropic ones, but with the particularity that the magnetic transfer functions are null with the repetition of the responses observed at different sites. An important feature is that when more than one anisotropy azimuth is involved, the impedance tensor is not diagonalizable at some periods and has a quasi-3D form. In $2 \mathrm{D}$, the responses are more or less complex depending on whether Maxwell's equations can be decoupled or not. Not so well known is the fact that in some situations the induction arrows can be null as well. For 3D anisotropic structures, the solutions 
take the general form. Even in the simplest cases, the responses do not fully characterize the conductivity tensor, making the solution to the inverse problem non-unique. Weidelt's statement that anisotropic responses can be explained with a 3D isotropic model has to be understood as a consequence of the lack of resolution at the investigation depth. This equivalence has been successfully applied to reproduce macro-anisotropic structures, but it should be taken carefully as even the simplest models (e.g. anisotropic half-space) cannot always be explained by a 3D model.

b) How to identify its footprint?

In the responses themselves, anisotropy can be identified by apparent inconsistencies between TE and TM responses and induction vectors, and by phases rolling out of their quadrant (PROQ). However, phases out of quadrant can also be explained by strong galvanic distortion, 2D structures with high resistivity contrasts, 3D conductive bodies generating strong current channelling or a superposition of different effects. An important limitation is that for realistic situations with weak anisotropy, these footprints are not evident.

Using dimensionality tools, it is possible to identify anisotropy, with some remarks. Results have to be processed in a statistical frame, and interpreted from the joint dimensionality analysis at different sites and periods (as opposed to isotropic dimensionality analysis). It is important that all the data components are used, including the tipper if possible, to solve possible ambiguities. Inconsistencies between different dimensionality tools (e.g. WALDIM, strike analysis) might indicate anisotropy as well.

Additionally, anisotropy can be identified in the results from data inversion: Unrealistic features can be identified as anisotropic artefacts, or a succession of conductive and resistive bodies can be labelled as macro-anisotropy.

c) How to proceed in real situations, with modelling codes and strategies for inversion? 
One should check if data are really anisotropic by looking for evidences from MT and other data: most of the MT studies advocating electrical anisotropy are supported by other anisotropic evidences (usually seismic anisotropy); and, rule out 3D effects: if 3D model responses cannot fit the full dataset (or if the 3D model is totally unrealistic), anisotropy might be needed to explain the responses.

In recent years, a great step has been taken with the development and introduction of new forward and anisotropic inversion codes. It is recommended to test the data with different codes. Even if structure is more complex, 1D inversion results can be used to construct 2D anisotropic models. However, the solution is not-unique, even in 1D. It is worth taking into account that anisotropy affects only sites above and surrounding, and hence, it is possible to model the rest of data using isotropic codes. Corroborations with other data are still necessary, and joint inversion can be the key to do so.

I will finish this review by mentioning that in the last 7 years a significant number of MT studies have included anisotropy in their results, with a wide range of interpretations for the causes of anisotropy, in most cases supported by other data.

\section{Acknowledgements}

I would like to thank the Program Committee of the 21 EMIW for the opportunity to present this review. It would not have been possible without the collaboration, encouragement and discussions with other colleagues, and those who kindly let me use their materials for the review. I would like to thank mainly Josef Pek and my colleagues at the EXES group in the Universitat de Barcelona, particularly Pilar Queralt and Juanjo Ledo. I wrote most of this review during my stay at Geomar, Helmholtz Centre for Ocean Research, in Kiel, Germany. For that I thank Marion Jegen and the people in the Marine EM group for the facilities offered and the useful discussions. I thank the Workshop Financial Committee, the project PIERCO2 (CGL2009-07604) and the Facultat de Geologia of the University of Barcelona for the financial support to attend the workshop. Finally I would like to thank the editor Graham Heinson, an anonymous referee and especially Alan G Jones who greatly helped improving the paper. 


\section{References}

Abramovici F (1974) The forward magnetotelluric problem for an inhomogeneous and anisotropic structure. Geophysics 39:56-68. doi:10.1190/1.1440412

Abramovici F, Shoham Y (1977) Inversion of anisotropic magnetotelluric data. Geophys J R Astr Soc 50:55-74. doi:10.1111/j.1365-246X.1977.tb01324.x

Baba K (2005) Electrical structure in marine tectonic settings. Surv Geophys 26:701-731. doi:10.1007/s10712-005-1831-2

Baba K, Chave AD, Evans RL, Hirth G, Mackie RL (2006) Mantle dynamics beneath the East Pacific Rise at $17^{\circ} \mathrm{S}$ : Insights from the Mantle Electromagnetic and Tomography (MELT) experiment. J Geophys Res 111:B02101. doi:10.1029/2004JB003598

Berdichevsky MN, Pushkarev PY (2006) Are the crustal and mantle conductive zones isotropic or anisotropic? Acta Geophys 54:333-342. doi:10.2478/s11600-006-0031-x

Boerner DE, Kurtz RD, Craven JA, Ross GM, Jones FW (2000) A synthesis of electromagnetic studies in the Lithoprobe Alberta Basement Transect: constraints on Paleoproterozoic indentation tectonics. Can J Earth Sci 37:1509-1534. doi:10.1139/e00-063

Brasse H, Kapinos G, Li Y, Mütschard L, Soyer W, Eydam D (2009) Structural electrical anisotropy in the crust at the South-Central Chilean continental margin as inferred from geomagnetic transfer functions. Phys Earth Planet Int 173:7-16. doi:10.1016/j.pepi.2008.10.017

Caldwell TG, Bibby HM, Brown C (2004) The magnetotelluric phase tensor. Geophys J Int 158:457469. doi:10.1111/j.1365-246X.2004.02281.x

Carcione JM, Ursin B, Nordskag JI (2007) Cross-property relations between electrical conductivity and the seismic velocity of rocks. Geophysics 72:E193-E204. doi:10.1190/1.2762224

Caricchi L, Gaillard F, Mecklenburgh J, Le Trong E (2011) Experimental determination of electrical conductivity during deformation of melt-bearing olivine aggregates: Implications for electrical anisotropy in the oceanic low velocity zone. Earth Planet Sci Lett 302:81-94. doi:10.1016/j.epsl.2010.11.041

Chen X, Weckmann U (2012) Inversion of 2D magnetotelluric data with anisotropic conductivities.21 Electromagnetic Induction Workshop, Darwin, Australia

Chen X, Weckmann U, Tietze K (2009) From forward modelling of MT phases over $90^{\circ}$ towards 2D anisotropic inversion. Schmucker-Weidelt-Colloquium für Elektromagnetische Tiefenforschung, Heimvolkshochschule am Seddiner See. Germany

Chouteau M, Tournerie B (2000) Analysis of magnetotelluric data showing phase rolling out of quadrant (PROQ). $70^{\text {th }}$ Annual Int Meeting, SEG Expanded Abstracts 19:344-46. http://link.aip.org/link/doi/10.1190/1.1816062

Constable SC, Parker RL, Constable CG (1987) Occam's inversion: A practical algorithm for generating smooth models from electromagnetic sounding data. Geophysics 52:289-300. doi:10.1190/1.1442303 
Davydycheva S, Druskin V, Habashy T (2003) An efficient finite-difference scheme for electromagnetic logging in 3D anisotropic inhomogeneous media. Geophysics 68:1525-1536. doi:10.1190/1.1620626

Davydycheva S, Wang T (2011) A fast modelling method to solve Maxwell's equations in 1D layered biaxial anisotropic medium. Geophysics 76:F293-F302. doi:10.1190/GEO2010-0280.1

Dekker DL, Hastie LM (1980) Magneto-telluric impedances of an anisotropic layered Earth model. Geophys J R Astr Soc 61:11-20. doi:10.1111/j.1365-246X.1980.tb04300.x

Eaton DW, Jones AG (2006) Tectonic fabric of the subcontinental lithosphere: Evidence from seismic, magnetotelluric and mechanical anisotropy. Phys Earth Planet Int 158:85-91. doi:10.1016/j.pepi.2006.05.005

Edwards N (2005) Marine controlled source electromagnetics: principles, methodologies, future commercial applications. Surv Geophys 26:675-700. doi:10.1007/s10712-005-1830-3

Evans RL, Jones, AG, Garcia X, Muller M, Hamilton M, Evans S, Fourie CJS, Spratt J, Webb S, Jelsma H, Hutchins D (2011) Electrical lithosphere beneath the Kaapvaal craton, southern Africa, J Geophys Res 116:B04105. doi:10.1029/2010JB007883

Evans RL, Tarits P, Chave AD, White A, Heinson G, Filloux JH, Toh H, Seama N, Utada H, Booker JR, Unsworth MJ (1999) Asymmetric electrical structure in the mantle beneath the East Pacific Rise at $17^{\circ} \mathrm{S}$. Science 286:752-756. doi:10.1126/science.286.5440.752

Everett ME (2012) Theoretical developments in electromagnetic induction geophysics with selected applications in the near surface. Surv Geophys 33:29-63. doi:10.1007/s10712-011-9138-y

Frederiksen AW, Ferguson IJ, Eaton D, Miong SK, Gowan E (2006) Mantle fabric at multiple scales across an Archean-Proterozoic boundary, Grenville Front, Canada. Phys Earth Planet Int 158:240-263. doi:10.1016/j.pepi.2006.03.025

Gaillard F, Malki M, Iacono-Marziano G, Pichavant M, Scaillet B (2008) Carbonatite melts and electrical conductivity in the asthenosphere. Science 322:1363-1365. doi:10.1126/science.1164446

Gatzemeier A, Moorkamp M (2005) 3D modelling of electrical anisotropy from electromagnetic array data: hypothesis testing for different upper mantle conduction mechanisms. Phys Earth Planet Int 149:225-242. doi:10.1016/j.pepi.2004.10.004

Gatzemeier A, Tommasi A (2006) Flow and electrical anisotropy in the upper mantle: Finite-element models constraints on the effects of olivine crystal preferred orientation and microstructure. Phys Earth Planet Int 158:92-106. doi:10.1016/j.pepi/2006.01.009

Hamilton MP, Jones AG, Evans RL, Evans S, Fourie CJS, Garcia X, Mountford A, Spratt JE, SAMTEX team (2006) Electrical anisotropy of South African lithosphere compared with seismic anisotropy from shear-wave splitting analyses. Phys Earth Planet Int 158:226-239. doi:10.1016/j.pepi.2006.03.027

Häuserer M, Junge A (2011) Electrical mantle anisotropy and crustal conductor: a 3-D conductivity model of the Rwenzori Region in western Uganda. Geophys J Int 185: 1235-1242. doi:10.1111/j.1365-246X.2011.05006.x 
Heinson G, White A (2005) Electrical resistivity of the Northern Australian lithosphere: Crustal anisotropy or mantle heterogeneity? Earth Planet Sci Lett 232:157-170. doi:10.1016/j.eps1.2004.12.029

Heise W, Caldwell TG, Bibby HM, Brown C (2006) Anisotropy and phase splits in magnetotellurics. Phys Earth Planet Int 158:107-121. doi:10.1016/j.pepi.2006.03.021

Heise W, Pous J (2001) Effects of anisotropy on the two-dimensional inversion procedure. Geophys J Int 147:610-621. doi:10.1046/j.0956-540x.2001.01560.x

HeiseW, Pous J (2003) Anomalous phases exceeding $90^{\circ}$ in magnetotellurics: anisotropic model studies and a field example. Geophys J Int 155:308-318. doi:10.1046/j.1365246X.2003.02050.X

Hou J, Mallan RK, Torres-Verdín C (2006) Finite-difference simulation of borehole EM measurements in 3D anisotropic media using coupled scalar-vector potentials. Geophysics 71:G225-G223. doi:10.1190/1.2245467

Ichihara H, Mogi T (2009) A realistic 3-D resistivity model explaining anomalous large magnetotelluric phases: the L-shaped conductor model. Geophys J Int 179:14-17. doi:10.1111/j.1365-246X.2009.04310.x

Jelínek V (1981) Characterization of the magnetic fabric of rocks. Tectonophysics 79:T63-T67. doi:10.1016/0040-1951(81)90110-4

Jones AG (2006) Electromagnetic interrogation of the anisotropic Earth: Looking into the Earth with polarized spectacles. Phys Earth Planet Int 158:281-291. doi:10.1016/j.pepi.2006.03.026

Jones AG (2012a) Distortion decomposition of the magnetotelluric impedance tensors from a onedimensional anisotropic Earth. Geophys J Int 189:268-284. doi:10.1111/j.1365246X.2012.05362.X

Jones AG (2012b) Distortion of magnetotelluric data: its identification and removal. In: Chave AD, Jones AG (eds) The magnetotelluric method: Theory and practice. Cambridge Univ Press, pp 219-302

Jones AG, Evans RL, Eaton DW (2009a) Velocity-conductivity relationships for mantle mineral assemblages in Archean cratonic lithosphere based on a review of laboratory data and HashinShtrikman extremal bounds. Lithos 109:131-143. doi:10.1016/j.lithos.2008.10.014

Jones AG, Evans RL, Muller MR, Hamilton MP, Miensopust MP, Garcia X, Cole P, Ngwisanyi T, Hutchins D, Fourie CJS, Jelsma H, Aravanis T, Pettit W, Webb S, Wasborg J, SAMTEX Team (2009b) Area selection for diamonds using magnetotellurics: Examples from southern Africa. Lithos112S:83-92. doi:10.1016/j.lithos.2009.06.011

Key K (2012) Marine electromagnetic studies of seafloor resources and tectonics. Surv Geophys 33:135-167. doi:10.1007/s10712-011-9139-х

Key K, Ovall J (2011) A parallel goal-oriented adaptive finite element method for 2.5-D electromagnetic modelling. Geophys J Int 186:137-154. doi:10.1111/j.1365-

246X.2011.05025.X

Kong FN, Johnstad SE, Røsten T, Westerdahl H (2008) A 2.5D finite-element-modeling difference method for marine CSEM modeling in stratified anisotropic media. Geophysics 73:F9-F19. doi:10.1190/1.2819691 
Kováčiková S, Pek J (2002) Generalized Riccati equations for 1-D magnetotelluric impedances over anisotropic conductors Part I: Plane wave field model. Earth Planets Space 54:473-482

Le Pape F, Jones AG, Vozar J, Wei W (2012) Penetration of crustal melt beyond the Kunlun Fault into northern Tibet. Nature Geoscience 5:330-335. doi:10.1038/NGEO1449

Ledo J (2006) 2-D versus 3-D magnetotelluric data interpretation. Surv Geophys 27:111-148. doi:10.1007/s10712-005-1757-8

Leibecker J, Gatzemeier A, Hönig M, Kuras O, Soyer W (2002) Evidence of electrical anisotropic structures in the lower crust and the upper mantle beneath the Rhenish Shield. Earth Planet Sci Lett 202:289-302. doi:10.1016/S0012-821X(02)00783-5

Lezaeta P, Haak V (2003) Beyond magnetotelluric decomposition: Induction, current channeling, and magnetotelluric phases over 90. J Geophys Res 108:B6, 2305. doi:10.1029/2001JB000990

Li X, Oskooi B, Pedersen LB (2000) Inversion of controlled-source tensor magnetotelluric data for a layered earth with azimuthal anisotropy. Geophysics 65:452-464. doi:10.1190/1.1444739

Li X, Pedersen LB (1991) The electromagnetic response of an azimuthally anisotropic half-space. Geophysics 56:1462-1473. doi:10.1190/1.1443166

Li X, Pedersen LB (1992) Controlled-source tensor magnetotelluric responses of a layered earth with azimuthal anisotropy. Geophys J Int 111:91-103. doi:10.1111/j.1365-246X.1992.tb00557.x

Li Y (2002) A finite-element algorithm for electromagnetic induction in two-dimensional anisotropic conductivity structures. Geophys J Int 148:389-401. doi:10.1046/j.1365-246x.2002.01570.x

Li Y, Dai S (2011) Finite element modelling of marine controlled-source electromagnetic responses in two-dimensional dipping anisotropic conductivity structures. Geophys J Int 185:622-636. doi:10.1111/j.1365-246X.2011.04974.X

Li Y, Luo M, Pei J (2012) A 2.5D finite element modelling of marine CSEM fields in arbitrarily anisotropic media. 21 Electromagnetic Induction Workshop, Darwin, Australia

Li Y, Pek J (2008) Adaptive finite element modelling of two-dimensional magnetotelluric fields in general anisotropic media. Geophys J Int 175:942-954. doi:10.1111/j.1365-

246X.2008.03955.x

Li Y, Pek J, Brasse H (2003) Magnetotelluric inversion for 2D anisotropic conductivity structures. In: Jordt A, Stoll J (eds) Protokoll uber das 20 Kolloquium Elektromagnetische Tiefenforschung, Königstein, Germany, pp 250-259

Li Y, Spitzer K (2005) Finite element resistivity modelling for three-dimensional structures with arbitrary anisotropy. Phys Earth Planet Int 150:15-27. doi:10.1016/j.pepi.2004.08.014

Lilley FEM, Weaver JT (2010) Phases greater than $90^{\circ}$ in MT data: Analysis using dimensionality tools. J App Geophys 70:9-16. doi:10.1016/j.appgeo.2009.08.007

Linde N, Pedersen LB (2004) Evidence of electrical anisotropy in limestone formations using the RMT technique. Geophysics 69:909-916. doi:10.1190/1.1778234

Loewenthal D, Landisman M (1973) Theory for magnetotelluric observations on the surface of a layered anisotropic half space. Geophys J R Astr Soc 35:195-214. doi:10.1111/j.1365246X.1973.tb02422.x

Løseth LO, Ursin B (2007) Electromagnetic fields in planarly layered anisotropic media. Geophys J Int 170:40-80. doi:10.1111/j.1365-246X.2007.03390.x 
Mackie RL (2002) User manual and software documentation for two-dimensional inversion of magnetotelluric data. Anisotropy Version 6.7. GSY-USA Inc. San Francisco, CA, USA

Mandolesi E, Jones AG (2012a) Magnetotelluric inversion based on mutual information. Geophys J Int. Submitted

Mandolesi E, Jones AG (2012b) Magnetotelluric inversion in a 2D anisotropic environment. EGU General Assembly

Mann JE (1965) The importance of anisotropic conductivity in magnetotelluric interpretation. J Geophys Res 70:2940-2942. doi:10.1029/JZ070i012p02940

Martí A (2006) A magnetotelluric investigation of geoelectrical dimensionality and study of the Central Betic crustal structure. Dissertation, Univ Barcelona

Martí A, Queralt P, Ledo J (2009) WALDIM: A code for the dimensionality analysis of magnetotelluric data using the rotational invariants of the magnetotelluric tensor. Comput Geosci 35:2295-2303. doi:10.1016/j.cageo.2009.03.004

Martí A, Queralt P, Ledo J, Farquharson C (2010) Dimensionality imprint of electrical anisotropy in magnetotelluric responses. Phys Earth Planet Int 182:139-151. doi:10.1016/j.pepi.2010.07.007

Martí A, Rosell O, Queralt P, Ledo J, Marcuello A, Roca E (2012) Are the geoelectric structures of the Betics lithosphere anisotropic? Insights from a complete dimensionality analysis of magnetotelluric data. EGU General Assembly

Martinelli P, Osella A (1997) MT forward modeling of 3-D anisotropic electrical conductivity structures using the Rayleigh-Fourier method. J Geomag Geoelectr 49:1499-1518. http://dx.doi.org/10.5636/jgg.49.1499

Matsuno T, Seama N, Evans RL, Chave AD, Baba K, White A, Goto T, Heinson G, Boren G, Yoneda A, Utada H (2010) Upper mantle electrical resistivity structure beneath the central Mariana subduction system, Geochem Geophys Geosyst 11:Q09003. doi:10.1029/2010GC003101

McNeice GW, Jones AG (2001) Multisite, multifrequency tensor decomposition of magnetotelluric data. Geophysics 66:158-173. doi:10.1190/1.1444891

Miensopust MP, Jones AG (2011) Artefacts of isotropic inversion applied to magnetotelluric data from an anisotropic Earth. Geophys J Int 187:677-689. doi:10.1111/j.1365-246X.2011.05157.x

Montahaie M, Oskooi B (2012) Magnetotelluric inversion for azimuthally anisotropic resistivities employing artificial neural networks. Acta Geophys. Submitted

Moorkamp M, Jones AG, Fishwick S (2010) Joint inversion of receiver functions, surface wave dispersion, and magnetotelluric data. J Geophys Res 115:B04318. doi:10.1029/2009JB006369

Naif S, Key K, Constable S, Evans R (2013) Melt-rich channel observed at the lithosphereasthenosphere boundary. Nature. In Press

Negi JG, Saraf PD (1972) Effect of anisotropy of the earth on the impedance measurements. Pure Appl Geophys 96:37-44. doi:10.1007/BF00875626

Negi JG, Saraf PD (1973) Inductive sounding of a stratified earth with transition layer resting on dipping anisotropic beds. Geophys Prospect 21:635-647. doi:10.1111/j.13652478.1973.tb00049.x

Neves AS (1957) The generalized magneto-telluric method. Dissertation, Mass Inst Technol 
Newman GA, Commer M, Carazzone JJ (2010) Imaging CSEM data in the presence of electrical anisotropy. Geophysics 75:F51-F61. doi:10.1190/1.3295883

Nover G (2005) Electrical properties of crustal and mantle rocks - a review of laboratory measurements and their explanation. Surv Geophys 26:593-651. doi:10.1007/s10712-0051759-6

O’Brien DP, Morrison HF (1967) Electromagnetic fields in an n-layer anisotropic half-space. Geophysics 32:668-677. doi:10.1190/1.1439882

Osella AM, Martinelli P (1993) Magnetotelluric response of anisotropic 2-D structures. Geophys J Int 115:819-828. doi:10.1111/j.1365-246X.1993.tb01494.x

Padilha AL, Vitorello I, Pádua MB, Bologna MS (2006) Lithospheric and sublithospheric anisotropy beneath central-southeastern Brazil constrained by long period magnetotelluric data. Phys Earth Planet Int 158:190-209. doi:10.1016/j.pepi.2006.05.006

Pankratov OV, Kuvshinov AV, Avdeev DB (1997) High-performance three-dimensional electromagnetic modelling using modified Neumann series. Anisotropic earth. J Geomag Geoelectr 49:1541-1547. http://dx.doi.org/10.5636/jgg.49.1541

Parkinson, WD (1962) The influence of continents and oceans on geomagnetic variations, Geophys J R Astron Soc 6:441-449. doi:10.1111/j.1365-246X.1962.tb02992.x

Patro BPK, Brasse H, Sarma SVS, Harinarayana T (2005) Electrical structure of the crust below the Deccan Flood Basalts (India), inferred from magnetotelluric soundings. Geophys J Int 163:931-943.doi:10.1111/j.1365-246X.2005.02789.X

Pavan Kumar G, Manglik A (2012). Electrical anisotropy in the Main Central Thrust Zone of the Sikkim Himalaya: inference from anomalous MT phase. J Asian Earth Sci 57:120-127. http://dx.doi.org/10.1016/j.jseaes.2012.06.017

Pek J (2009) Effects of electrical anisotropy upon magnetotelluric data: modelling and experiments. In: Spichak VV (ed) Modern methods of electromagnetic data measurement, processing and interpretation. Librokom Publ, Moscow, pp 110-135 (in Russian)

Pek J, Santos FAM (2002) Magnetotelluric impedances and parametric sensitivities for 1-D anisotropic layered media. Comput Geosci 28:939-950. doi:10.1016/S0098-3004(02)00014-6

Pek J, Santos FAM (2006) Magnetotelluric inversion for anisotropic conductivities in layered media. Phys Earth Planet Int 158:139-158. doi:10.1016/j.pepi.2006.03.023

Pek J, Santos FAM, Li Y (2008) Anomalies of magnetotellurics field due to electrical anisotropy and their inverse conductivity images. 19 EM Induction Workshop, Beijing, China

Pek J, Santos FAM, Li Y (2011) Non-linear conjugate gradient magnetotelluric inversion for 2-D anisotropic conductivities. Schmucker-Weidelt-Colloquium. Neustadt and der Weinstrasse, Germany

Pek J, Verner, T (1997) Finite-difference modelling of magnetotelluric fields in two-dimensional anisotropic media. Geophys J Int 128:505-521. doi:10.1111/j.1365-246X.1997.tb05314.X

Plotkin VV (2012) Inversion of heterogeneous anisotropic magnetotelluric responses. Russ Geol Geophys 53:829-836. doi:10.1016/j.rgg.2012.06.010

Pommier A (2012) Interpretation of magnetotelluric results using laboratory measurements. Review presented at the 21th EM Induction Workshop. Darwin, Australia 
Pous J, Muñoz G, Heise W, Melgarejo JC, Quesada C (2004) Electromagnetic imaging of Variscan crustal structures in SW Iberia: the role of interconnected graphite. Earth Planet Sci Lett 217:435-450. doi:10.1016/S0012-821X(03)00612-5

Praus O, Petr V (1969) Magnetotelluric calculations for interaction of polarized fields with anisotropic layered media. Can J Earth Sci 6:759-769. doi:10.1139/e69-070

Qin L, Yang C, Chen K (2013) Quasi-analytic solution of 2-D magnetotelluric fields on an axially anisotropic infinite fault. Geophys J Int 192:67-74. doi:10.1093/gji/gss018

Ramananjaona C, MacGregor L (2010) 2.5D inversion of CSEM data in a vertically anisotropic earth. Jour Phys: Conf Ser 255:012004. doi:10.1088/1742-6596/255/1/012004

Ramananjaona C, MacGregor L, Andréis D (2011) Sensitivity and inversion of marine electromagnetic data in a vertically anisotropic stratified earth. Geophys Pros 59:341-360. doi:10.1111/j.13652478.2010.00919.x

Reddy IK, Rankin D (1971) Magnetotelluric effect of dipping anisotropies. Geophys Prospect 19:8497. doi:10.1111/j.1365-2478.1971.tb00586.x

Reddy IK, Rankin D (1975) Magnetotelluric response of laterally inhomogeneous and anisotropic media. Geophysics 40:1035-1045. doi:10.1190/1.1440579

Rödder A, Junge A (2012) Modelling of apparent resistivity tensors and magnetotellurics phase tensors on anisotropic structures. 21 Electromagnetic Induction Workshop, Darwin, Australia

Rodi W, Mackie RL (2001) Nonlinear conjugate gradients algorithm for 2-D magnetotelluric inversion. Geophysics 66:174-187. doi:10.1190/1.444893

Rosell O (2012) Application of the magnetotelluric method in complex geological systems. Dissertation. Univ Barcelona. In preparation

Roux E, Moorkamp M, Jones AG, Bischoff, M, Endrun B, Ledebev S, Meier T (2011) Joint inversion of long-period magnetotelluric data and surface-wave dispersion curves for anisotropic structure: Application to data from Central Germany. Geophys Res Lett 38:L05304. doi:10.1029/2010GL046358

Ruiz-Constán A, Galindo-Zaldivar J, Pedrera A, Arzate JA, Pous J, Anahnah F, Heise W, Santos FAM, Marín-Lechado C (2010) Deep deformation pattern from electrical anisotropy in an arched orogen (Betic Cordillera, western Mediterranean). Geology 38:731-734. doi:10.1130/G31144.1

Santos FAM, Mendes-Victor LA (2000) 1D anisotropic versus 2D isotropic media in magnetotellurics. Acta Geod Geoph Hung 35:49-61

Saraf PD, Negi JG, Červ V (1986) Magnetotelluric response of a laterally inhomogeneous anisotropic inclusion. Phys Earth Planet Int 43:196-198. doi:10.1016/0031-9201(86)90046-4

Sasaki Y (2011) Anisotropic, joint 3D inversion of marine CSEM and MT data. $81^{\text {st }}$ Annual Int Meeting, SEG Expanded Abstracts 30:547-550. doi:10.1190/1.3628141

Schmoldt JP, Jones AG (2012) A novel anisotropic inversion approach for magnetotelluric data from subsurfaces with oblique geoelectric strike directions. Geophys J Int, in preparation.

Selway K, Thiel S, Key K (2012) A simple 2-D explanation for negative phases in TE magnetotelluric data. Geophys J Int 188:945-958. doi:10.1111/j.1365-246X.2011.05312.x 
Shalivahan, Bhattacharya BB (2005) Electrical anisotropy of asthenosphere in a region of window to mantle underneath Eastern Indian Craton. Phys Earth Planet Int 152:43-61. doi:10.1016/j.pepi.2005.06.001

Shoham Y, Loewenthal D (1975) Matrix polynomial representation of the anisotropic magnetotelluric impedance tensor. Phys Earth Planet Int 11:128-138. doi:10.1016/0031-9201(75)90006-0

Simpson F, Tommasi A (2005) Hydrogen diffusivity and electrical anisotropy of a peridotite mantle. Geophys J Int 160:1092-1102. doi:10.1111/j.1365-246X.2005.02563.X

Sinha AK (1969) The magnetotelluric effect in an inhomogeneous and anisotropic Earth. Geoexploration 7:9-28

Wang D, Mookherjee M, Xu Y, Karato S (2006) The effect of water on the electrical conductivity of olivine. Nature 443:977-980. doi:10.1038/nature05256

Wang T, Fang S (2001) 3-D electromagnetic anisotropy modeling using finite differences. Geophysics 66:1386-1398. doi:10.1190/1.1486779

Wannamaker PE (2005) Anisotropy versus heterogeneity in continental solid Earth electromagnetic studies: Fundamental response characteristics and implications for physicochemical state. Surv Geophys 26:733-765. doi:10.1007/s10712-005-1832-1

Wannamaker PE, Hasterok DP, Johnston JM, Stodt JA, Hall DB, Sodergren TL, Pellerin L, Maris V, Doerner WM, Groenewold KA, Unsworth MJ (2008) Lithospheric dismemberment and magmatic processes of the Great Basin-Colorado Plateau transition, Utah, implied from magnetotellurics. Geochem Geophys Geosyst 9:Q05019. doi:10.1029/2007GC001886

Weaver JT, Agarwal AK, Lilley FEM (2000) Characterization of the magnetotelluric tensor in terms of its invariants. Geophys J Int 141:321-336. doi:10.1046/j.1365-246x.2000.00089.x

Weckmann U (2012) Making and breaking of a continent: Following the scent of geodynamic imprints of the African continent using electromagnetics. Surv Geophys 33:107-134. doi:10.1007/s10712-011-9147-x

Weckmann U, Ritter O, Chen X, Tietze C, de Wit M (2012) Magnetotelluric image linked to surface geology across the Cape Fold Belt, South Africa. Terranova 24:207-212. doi:10.1111/j.13653121.2011.01054.x

Weckmann U, Ritter O, Haak V (2003) A magnetotelluric study of the Damara Belt in Namibia 2. MT phases over $90^{\circ}$ reveal the internal structure of the Waterberg Fault/Omaruru Lineament. Phys Earth Planet Int 138:91-112. doi:10.1016/S0031-9201(03)00079-7

Weidelt P (1999) 3D conductivity models: implications of electrical anisotropy. In: Oristaglio M, Spies B (eds) Three-dimensional electromagnetics. SEG, pp 119-137

Weidelt P (2007) Guided waves in marine CSEM. Geophys J Int 171:153-176. doi:10.1111/j.1365246X.2007.03527.x

Weiss CJ, Newman GA (2002) Electromagnetic induction in a fully 3-D anisotropic earth. Geophysics 67:1104-1114. doi:10.1190/1.1500371

Weiss CJ, Newman GA (2003) Electromagnetic induction in a generalized 3D anisotropic earth, Part 2: The LIN preconditioner. Geophysics 68:922-930. doi:10.1190/1.1581044

Yin C (2000) Geoelectrical inversion for a one-dimensional anisotropic model and inherent nonuniqueness. Geophys J Int 140:11-23. doi:10.1046/j.1365-246x.2000.00974.x 
Yin C (2003) Inherent nonuniqueness in magnetotellurics inversion for 1D anisotropic models.

Geophysics 68:138-146. doi:10.1190/1.1543201

Yin C (2006) MMT forward modeling for a layered earth with arbitrary anisotropy. Geophysics 71:G115-G128. doi:10.1190/1.2197492

Yin C, Maurer HM (2001) Electromagnetic induction in a layered earth with arbitrary anisotropy. Geophysics 66:1405-1416. doi:10.1190/1.1487086

Yin C, Weidelt P (1999) Geoelectrical fields in a layered earth with arbitrary anisotropy. Geophysics 64:426-434. doi:10.1190/1.1444547

Yoshino T (2010) Laboratory electrical conductivity measurement of mantle minerals. Surv Geophys 31:163-206. doi:10.1007/s10712-009-9084-0

Yoshino T, Matsuzaki T, Yamashita S, Katsura T (2006) Hydrous olivine unable to account for conductivity anomaly at the top of the asthenosphere. Nature 443:973-976. doi:10.1038/nature05223 


\section{Figure captions:}

Fig. 1 Diagram of successive Euler rotations applied to generate any orientation of the anisotropic principal directions, using the anisotropy strike $\left(\alpha_{S}\right)$, dip $\left(\alpha_{D}\right)$, and slant $\left(\alpha_{L}\right)$ angles. From Martí et al. (2010).

Fig. 2 Cross section of a layered model with an anisotropic layer; resistivity and phase responses obtained at an arbitrary site in the model. Tippers vectors are null. Phases are represented using the $e^{-i w t}$ convention.

Fig. 3 Apparent resistivities and phases for three 2D models with a vertically anisotropic body. For each model, the responses are computed for different values of $\sigma_{z z}$ to illustrate its effect (both xy and yx phases are represented in the third quadrant). From Pek et al. (2008).

Fig. 4 A: A 2-D slab with dipping anisotropy in an isotropic homogeneous half-space with $\rho_{0}=1000 \mathrm{ohm} \cdot \mathrm{m}$. The conductivity tensor of the slab is given by the principal resistivities $\rho_{x} / \rho_{y} /$ $\rho_{z}=500 / 10 / 500 \mathrm{ohm} \cdot \mathrm{m}$ for varying dip angles $\beta$. B: Apparent resistivities for the model computed for various dip angles $\beta\left(\alpha_{D}\right)$ at $\mathrm{T}=10 \mathrm{~s}$ (top: $\rho_{x y}$, bottom: $\rho_{y x}$ ). Modified from $\operatorname{Li}(2002)$.

Fig. 5 2D models with their corresponding responses: induction vector maps (non-reversed, Wiese convention) and pseudosections of the xy (TE) and yx (TM) apparent resistivities and phases. A: isotropic model. B: anisotropic model with 2 anisotropic half-layers, both with the same value of $\rho_{x x} ; \mathrm{C}$ : anisotropic model with 2 anisotropic half-layers, with different values of $\rho_{x x}$ and the same values of $\rho_{y y}$ and $\rho_{z z}$.

Fig. 6 Top view of polar impedance diagrams and real induction arrows at different points of the surface of models A and B, represented below, for periods between $30 \mathrm{~s}$ and $3000 \mathrm{~s}$. Blue dashed arrows indicate the strike and anisotropy directions. Model A is an anisotropic half-layer inserted in an isotropic half-space. Model B contains an anisotropic layer underlain by an isotropic halfspace. Modified from Pek and Verner (1997).

Fig. 7 A: 2D anisotropic model for the South-Central Chilean margin that best fits the real induction arrows (shown on top of the model). B: Scheme of the influence of the anisotropic blocks and the ocean near and farther from the coast. P1: coast effect, P2: anisotropic layer, P3: vector addition of P1 and P2; P4: vector resulting from the anisotropic model. Modified from Brasse et al. (2009).

Fig. 8 Top and front view of a 2D model with an anisotropic block in an isotropic layered medium, and the responses (apparent resistivities and phases and polar diagrams) computed at the centre of the model along different directions $\left(0^{\circ}\right.$ is the structural strike and $30^{\circ}$ is the anisotropic strike of the block). Modified from Pek (2009).

Fig. 9 2D model with an anisotropic block underlain by an anisotropic layer, with different directions of the maximum conductivities (in this case, perpendicular), and the MT responses computed at 6 points at the surface (phases are represented using $e^{+i w t}$ convention, and yx phases are shifted $180^{\circ}$ ). From Heise and Pous (2003). 
Fig. 10 A: Synthetic responses of the model in Table 1. B: Responses after applying galvanic distortion and noise. C: Responses recovered using the parameters obtained from the 3D/1Danis parametrization. Modified from Jones (2012a).

Fig. 11 A: 1D model with a vertically anisotropic layer $(20 / 1000 / 20 \mathrm{ohm} \cdot \mathrm{m})$. B: xy and yx apparent resistivities and phases (xx and yy components are null). Phases are represented using $e^{+i w t}$ convention, where yx phase is shifted $180^{\circ}$. C: Map view of the phase tensor ellipses at different periods. The yx component is constant with the period, as there are no conductivity changes in this polarization. The xy component senses the changes from $1000 \mathrm{ohm} \cdot \mathrm{m}$ to $20 \mathrm{ohm} \cdot \mathrm{m}$ and again to $1000 \mathrm{ohm} \cdot \mathrm{m}$. From Heise et al. (2006).

Fig. 12 2D anisotropic (A) and isotropic (B) models and the corresponding graphical representation of the phase tensor ellipses and real induction arrows (C and D). From Heise et al. (2006).

Fig. 13 Left: cross sections of two one dimensional models with two anisotropic layers. Right: dimensionality pattern of the corresponding responses, with the strike angles indicated. From Martí et al. (2010).

Fig. 14 Dimensionality patterns corresponding to the responses of models 4a (model a in Fig. 12), $4 \mathrm{~b}$ (model b in Fig. 12), and model 4c, which is a variation of model 4a with anisotropy rotated $30^{\circ}$. Modified from Martí et al. (2010).

Fig. 15 Joint MT and seismic models: In black: A: Minimum (solid lines) and maximum (dashed lines) resistivities on a logarithmic scale, and electrical anisotropy azimuth, B: mean values of shear-wave velocities, azimuthal anisotropy coefficient and fast-propagation azimuth. Grey lines represent all the possible solutions fitting the datasets within error bars. Background colours represent, for a certain depth, the number of models within a given interval for each inversion parameter. Modified from Roux et al. (2011).

Fig. 16 Two-dimensional resistivity (A) isotropic and (B) anisotropic models obtained using the anisotropic inversion in this study and (C) model presented by Evans et al. (1999). The rise axis is located at $0 \mathrm{~km}$ on the horizontal axis, and triangles indicate the locations of the MT measurements. Modified from Baba et al. (2006).

Fig. 17 Inversion of noisy synthetic MT data generated by a model of a shallow, strongly anisotropic anomaly and a layer of moderate anisotropy increasing from the left to the right beneath the anomalous block. The panels show the principal resistivities (top), anisotropy strike and anisotropy ratio ( $\alpha_{\mathrm{S}}, v_{\text {ani }}$, bottom panels) recovered by the inversion. The bottom rightmost panel shows the geometry and parameters of the true model generating the data (resistivities are given in ohm.m). The regularization weights used are $\lambda_{\mathrm{s}}=10, \lambda_{\mathrm{a}}=3$. The inverse model's RMS is 1.060. Modified from Pek et al. (2011).

Fig. 18 Isotropic and anisotropic inversion results of the ODS part of the ZIM profile. From Miensopust and Jones (2011). 


\section{Table captions:}

Table 1 Summary of particular types of anisotropy, depending on the different conductivity values and geometry. For the uniaxial case, only examples in which the tensor is diagonal are shown.

Table 2 Dimensionality criteria extended to anisotropic structures, characterized by the WAL invariants criteria indicating isotropic 2D. Modified from Martí et al. (2010). 

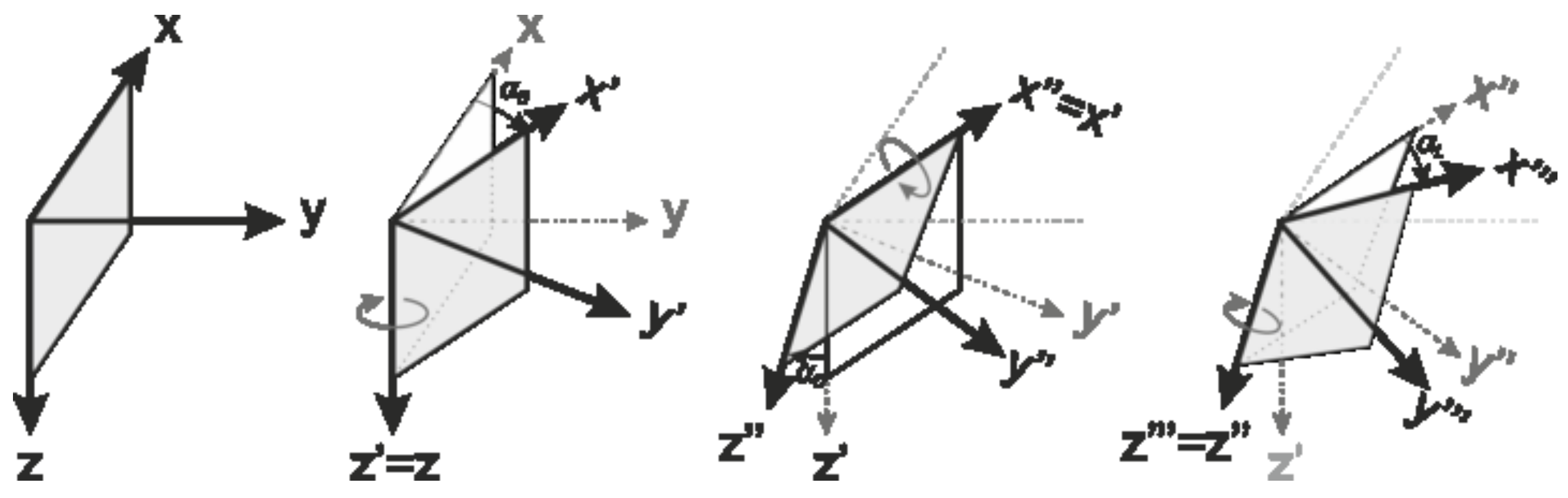

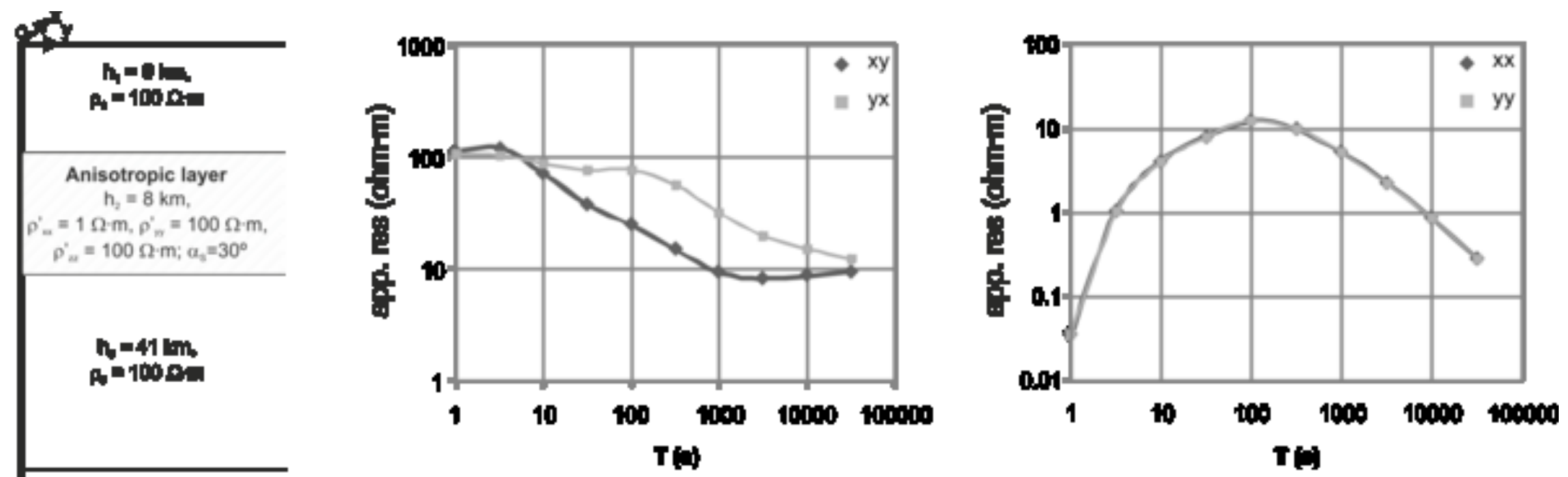

netom
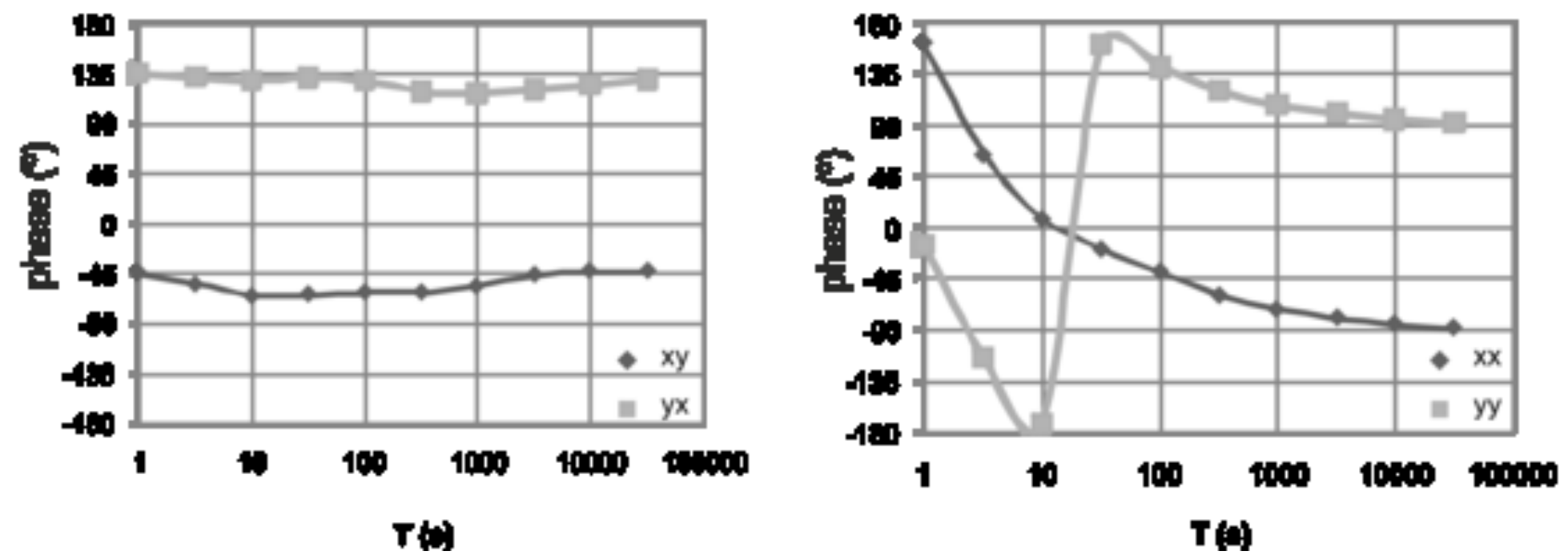

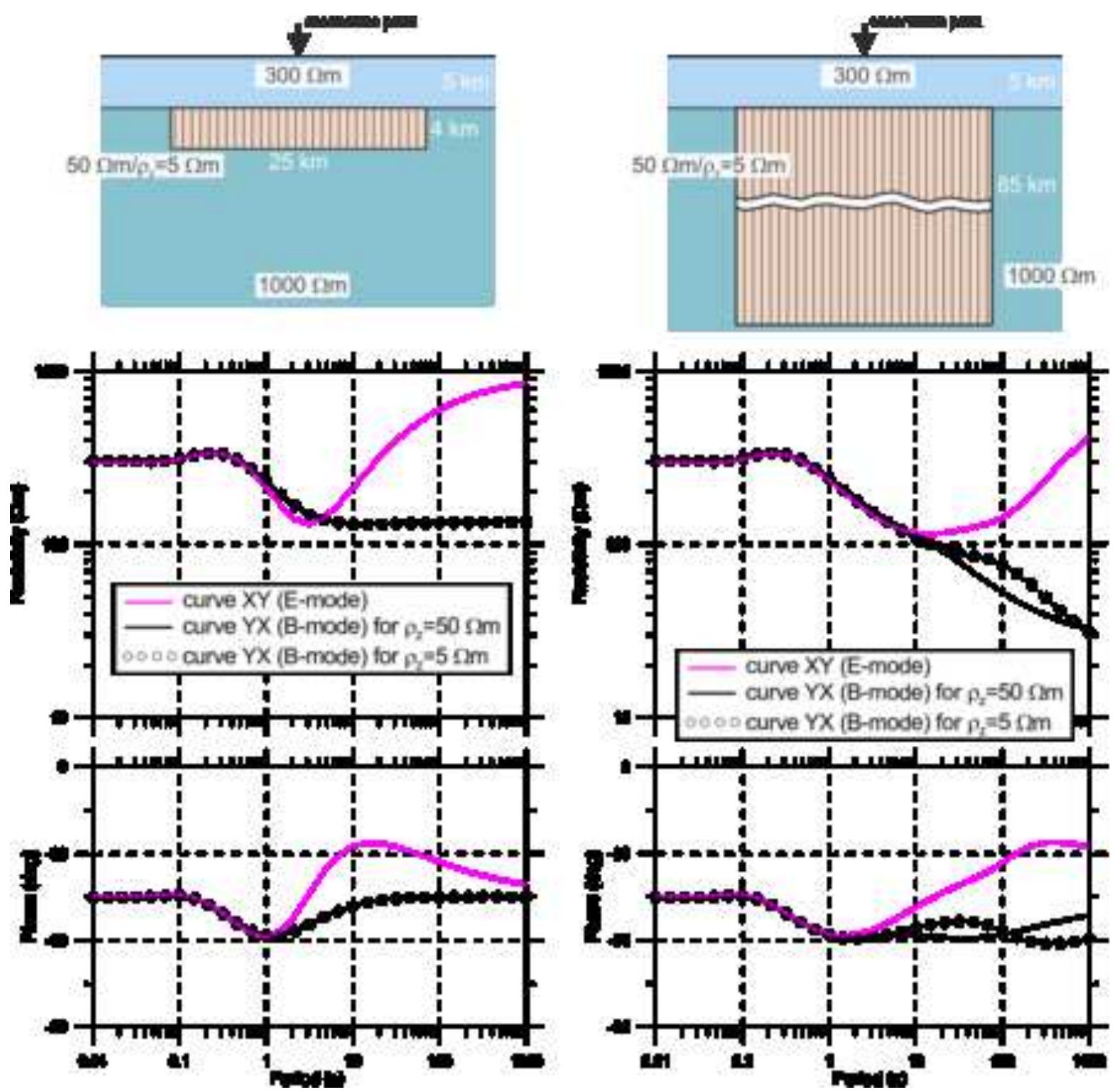

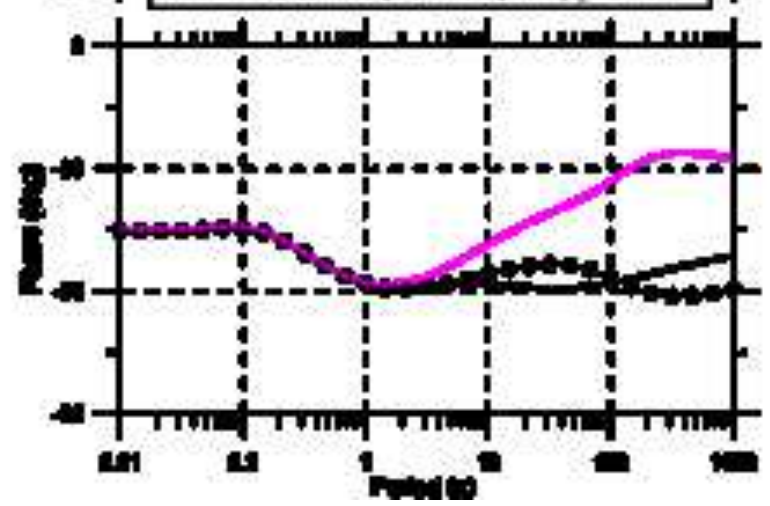

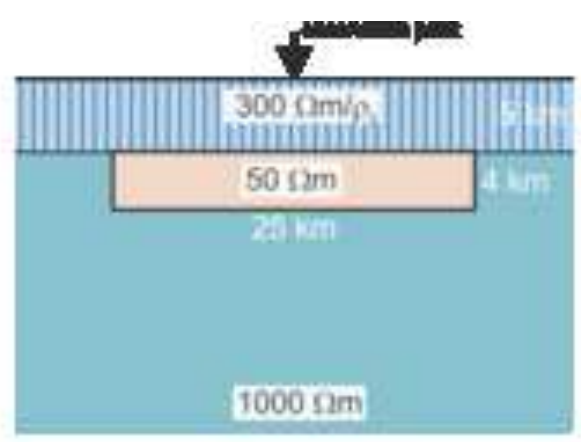

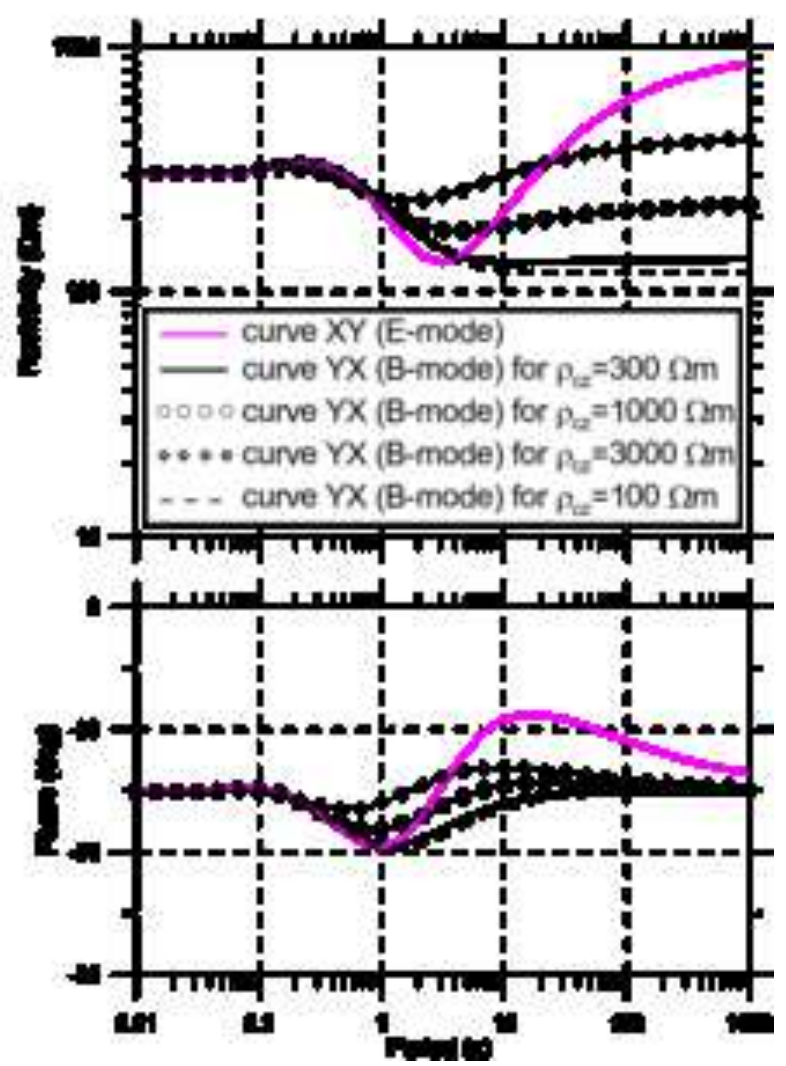




\section{Figure 4}

Click here to download high resolution image

A
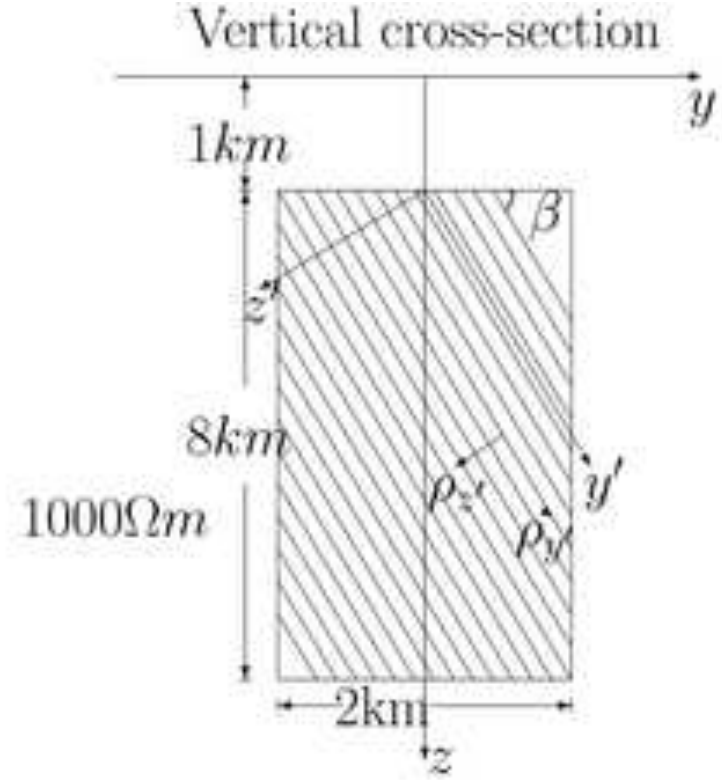

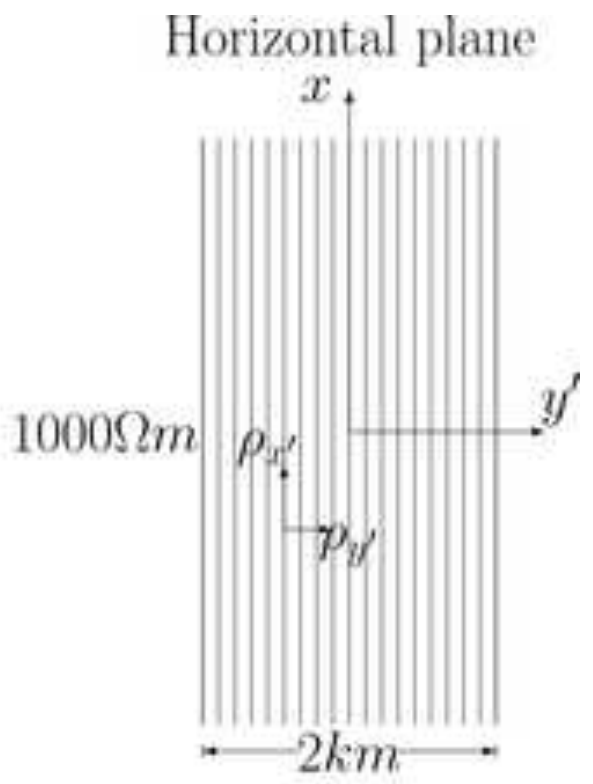

B
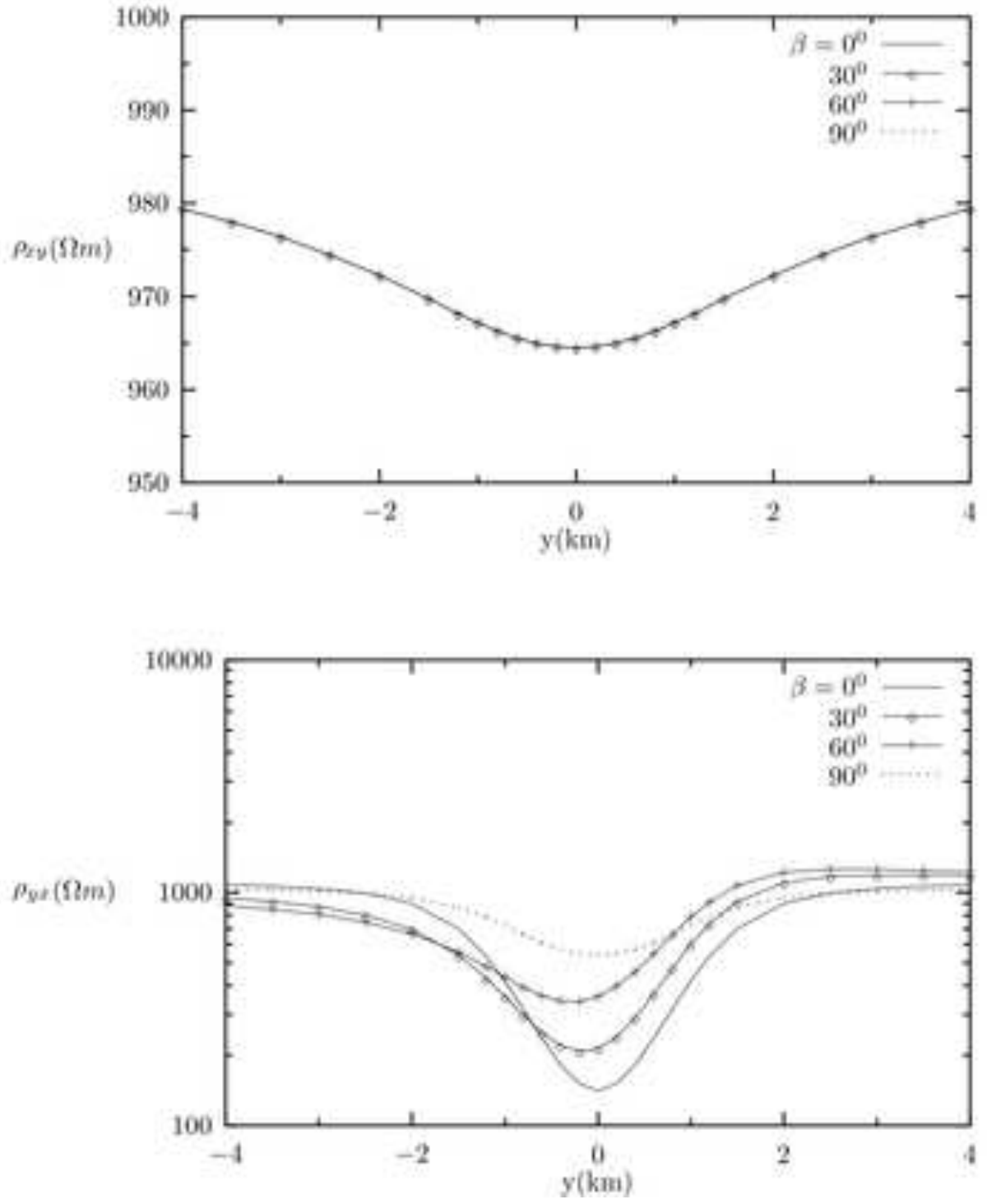


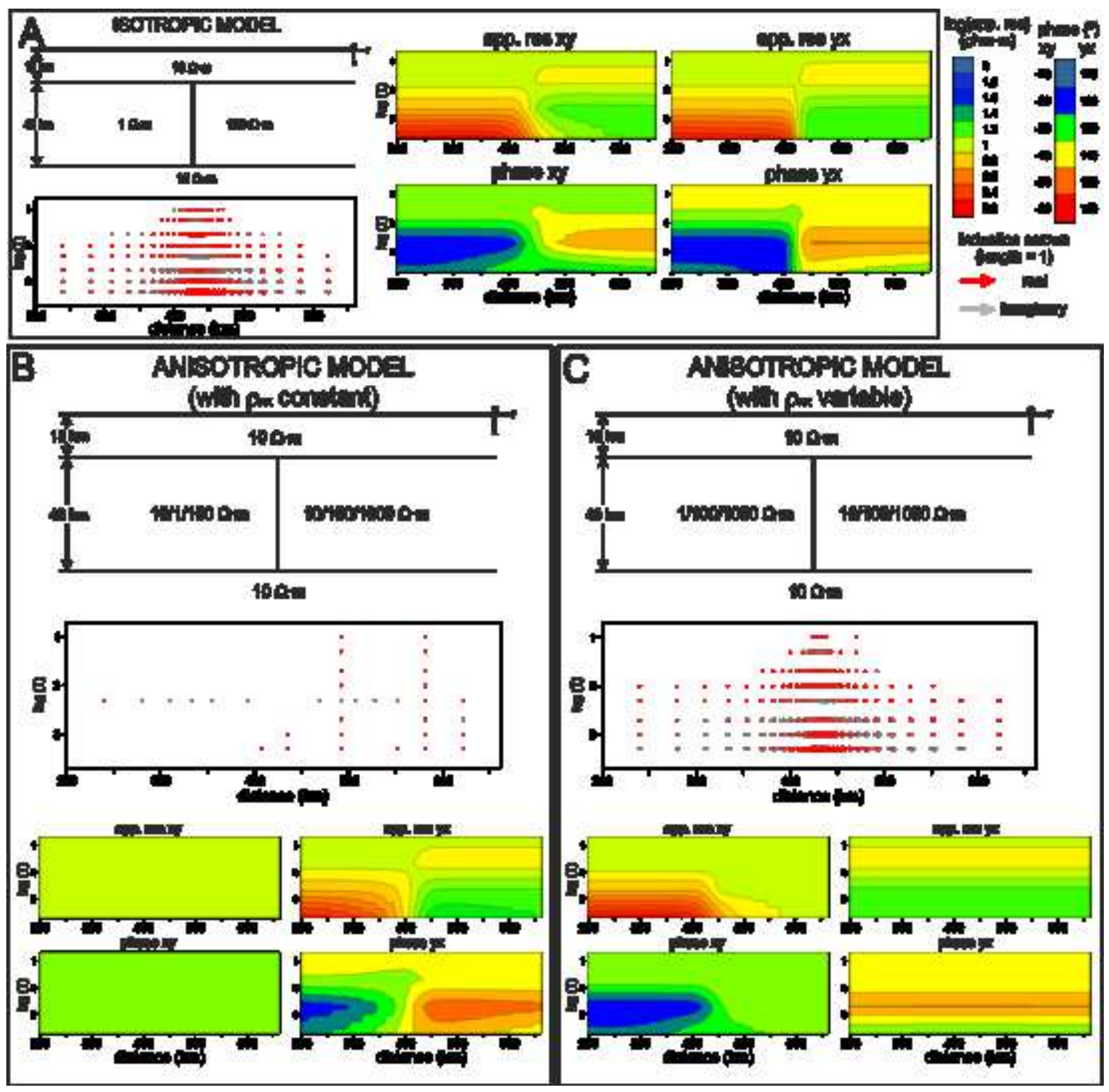


A

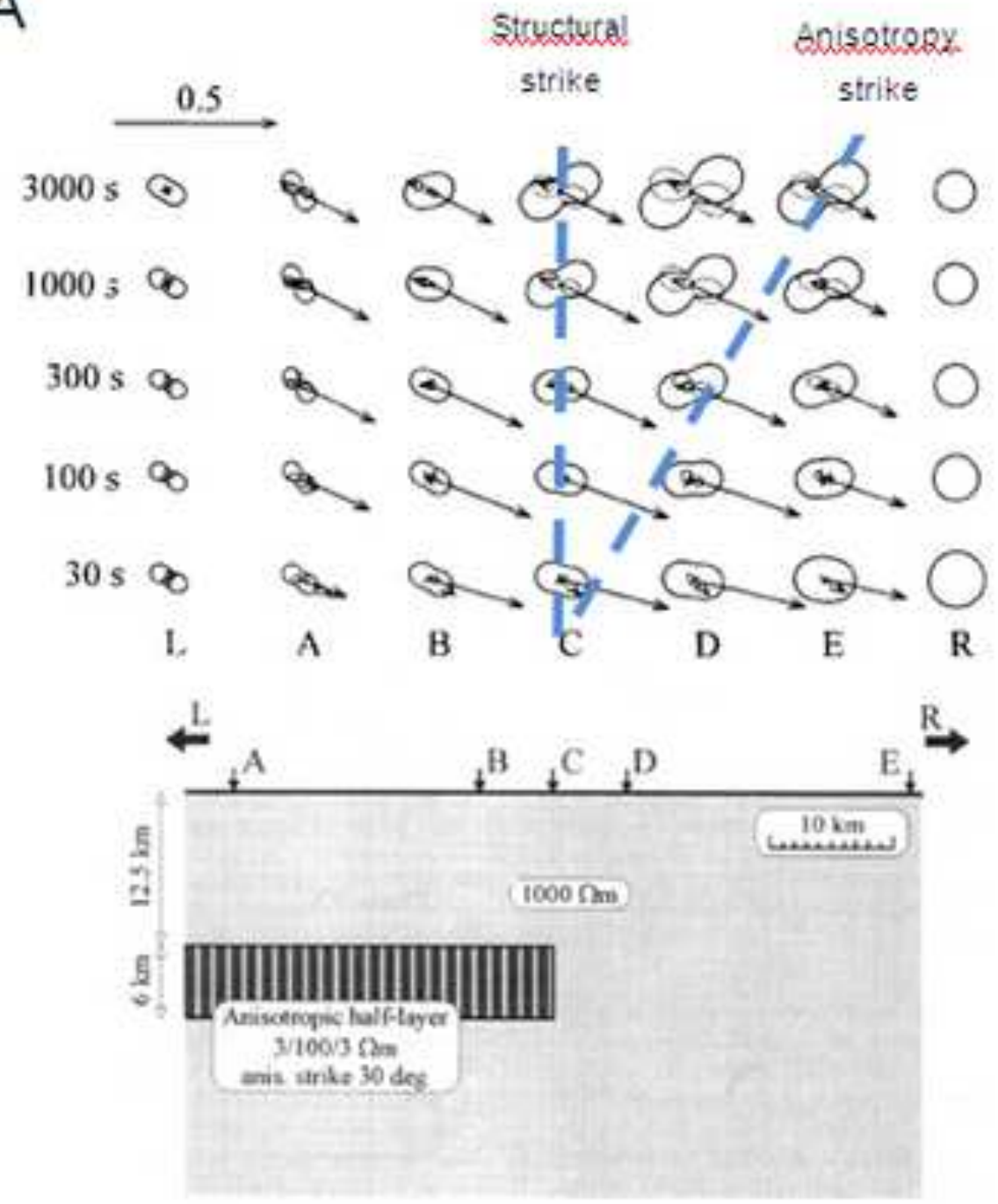

B

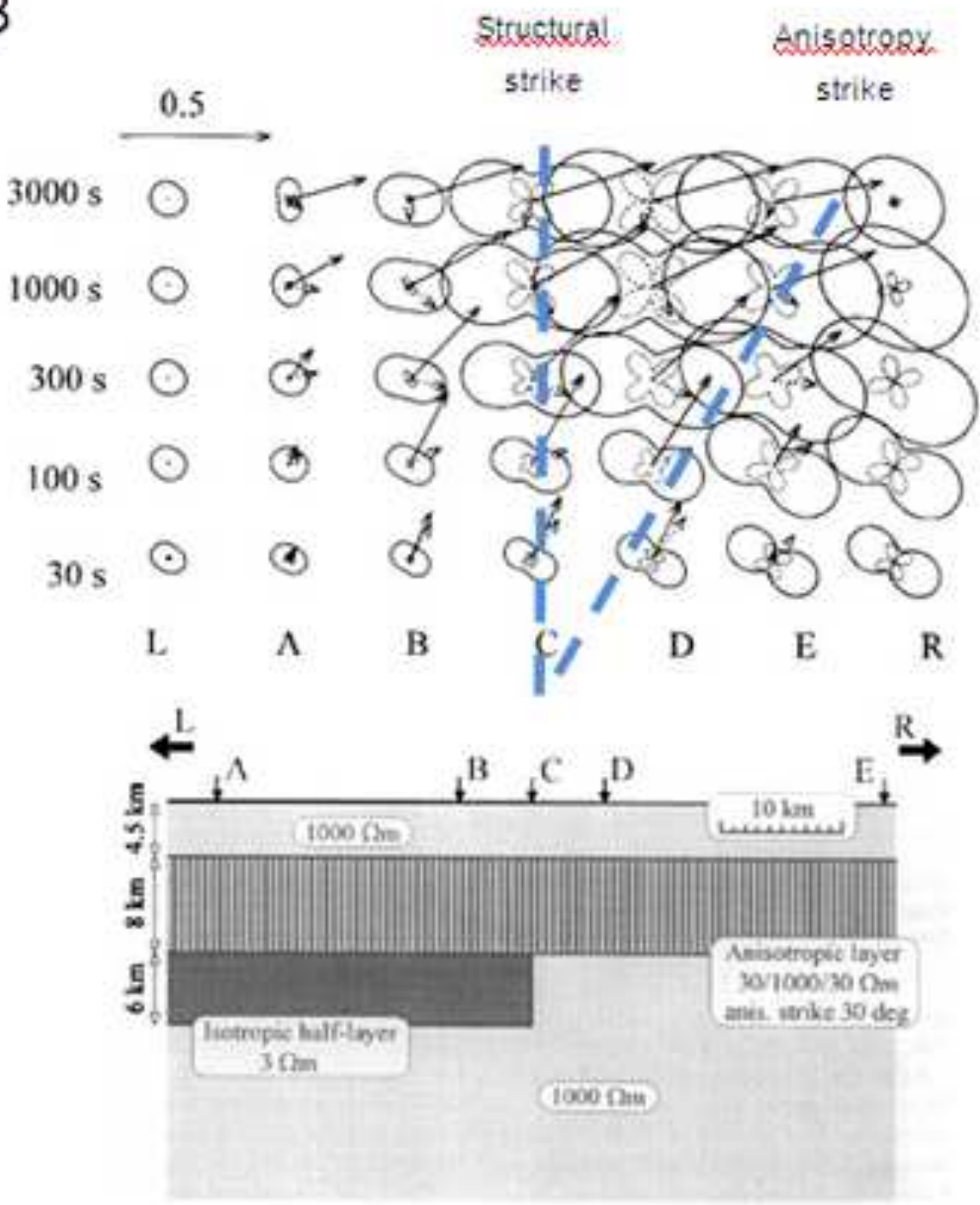


A

I: $10 / 500 / 10 \Omega \mathrm{m}, 45^{\circ}$ II: $5 / 500 / 5 \Omega \mathrm{m}, 38^{\circ}$

III: $1 / 500 / 1 \Omega \mathrm{m}, 50^{\circ}$
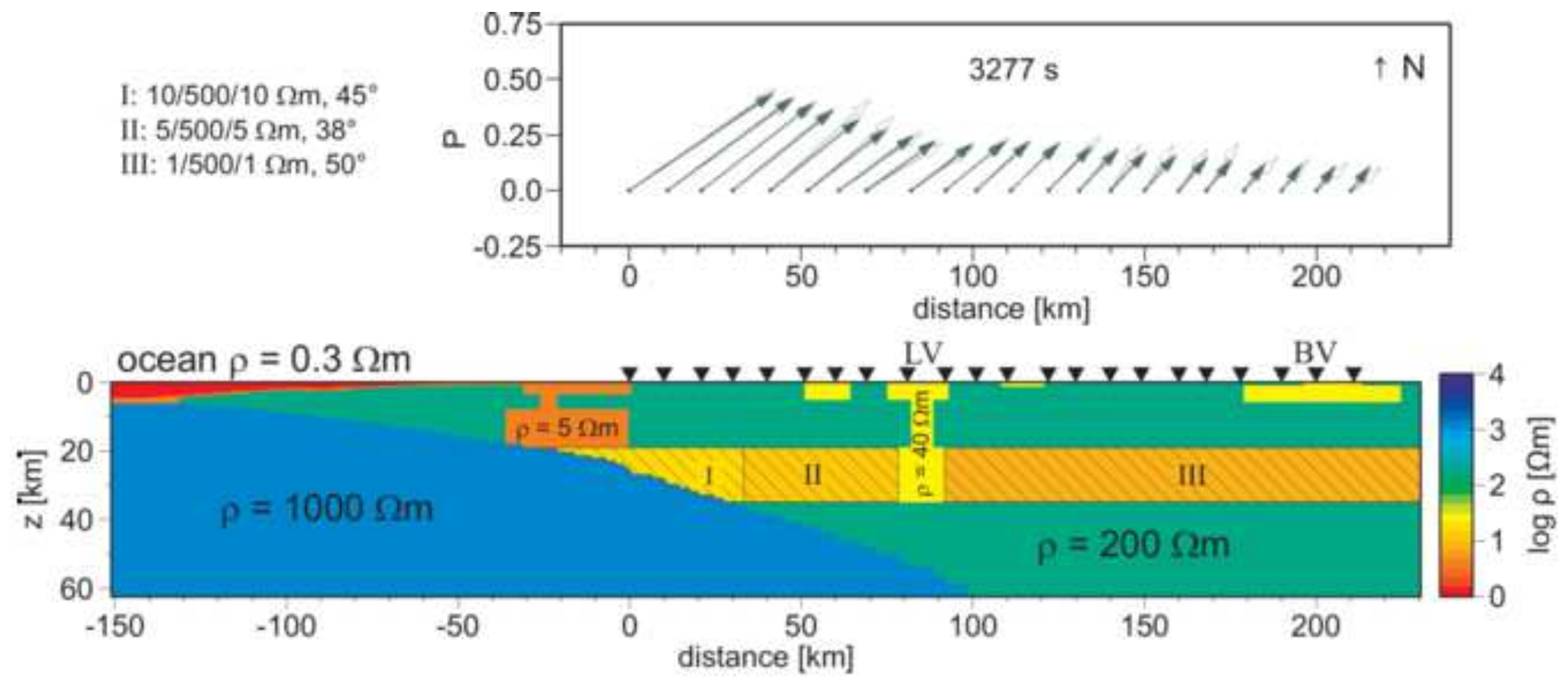

closer to coast

farther from coast
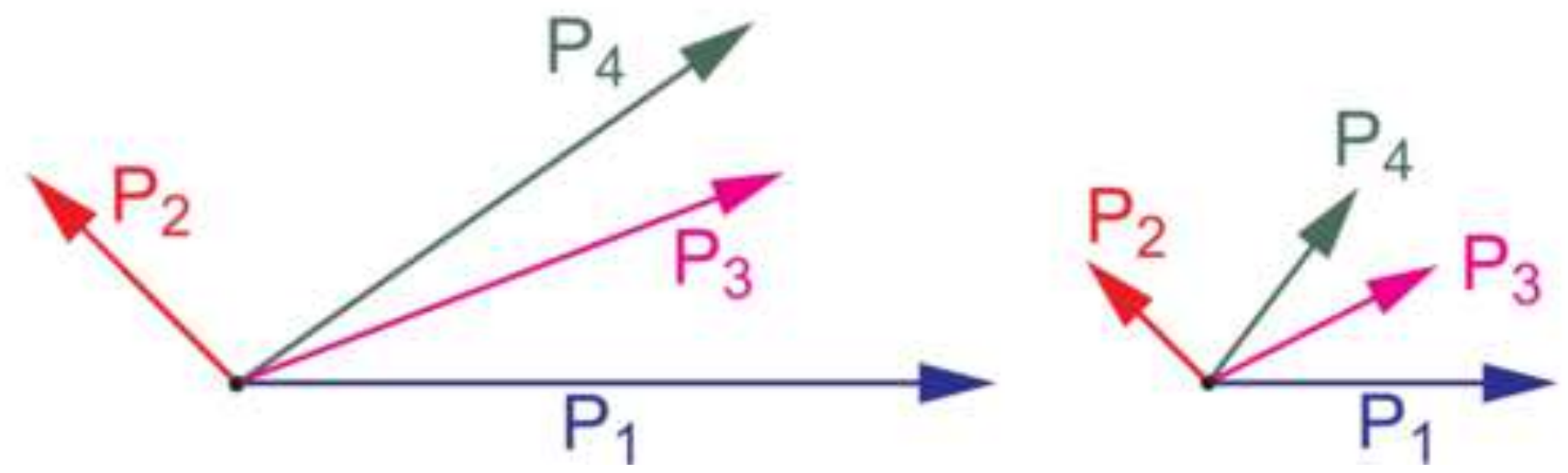


\section{Shallow now-enfiace enloobleple block}
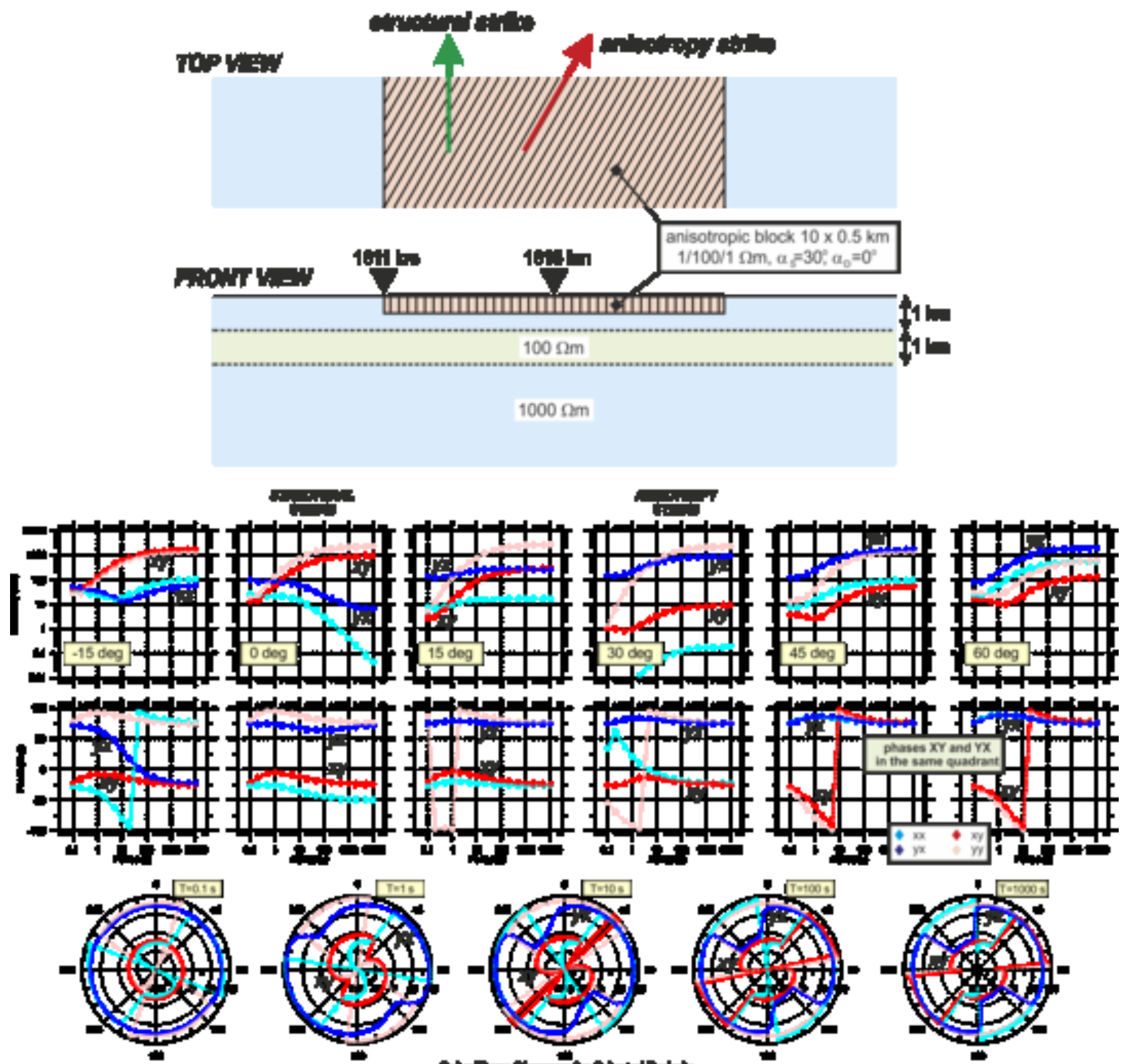

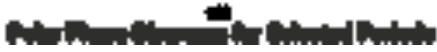
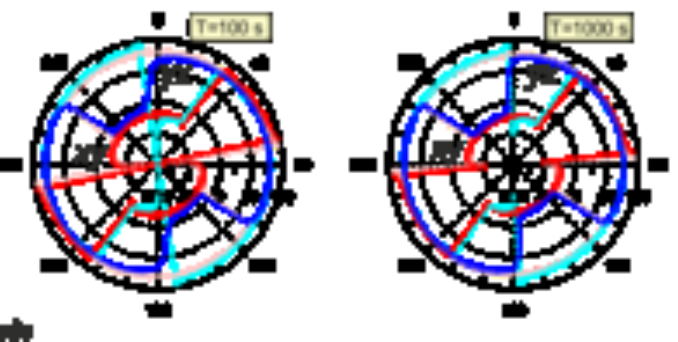

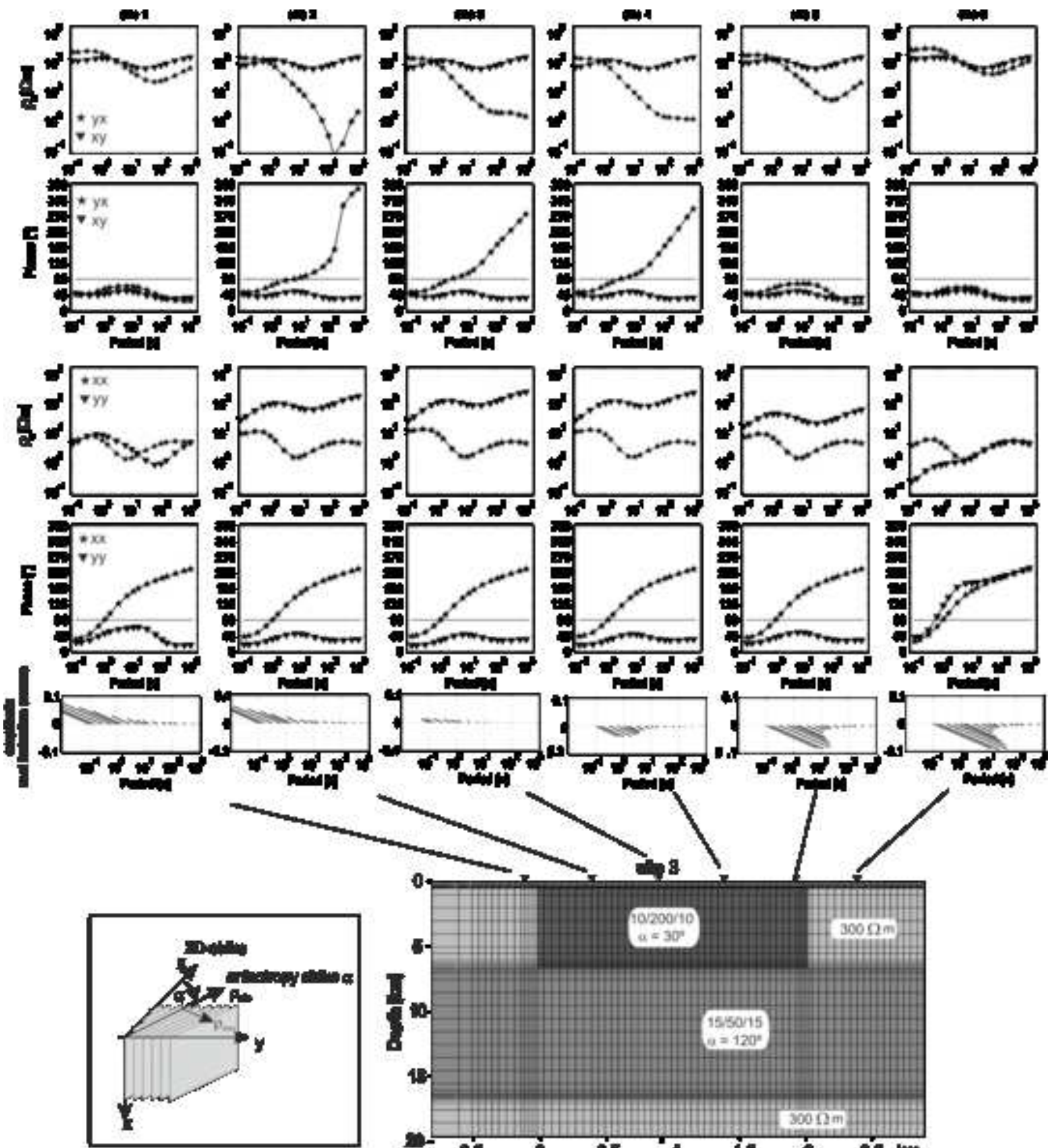

())

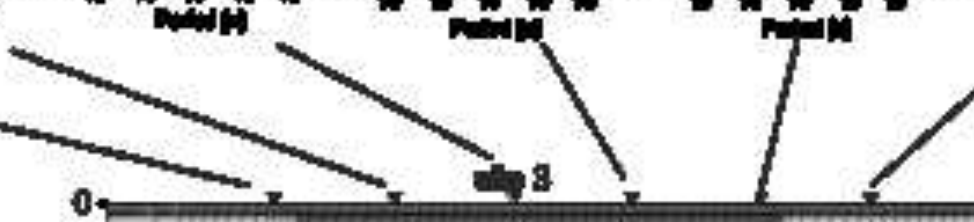

nim

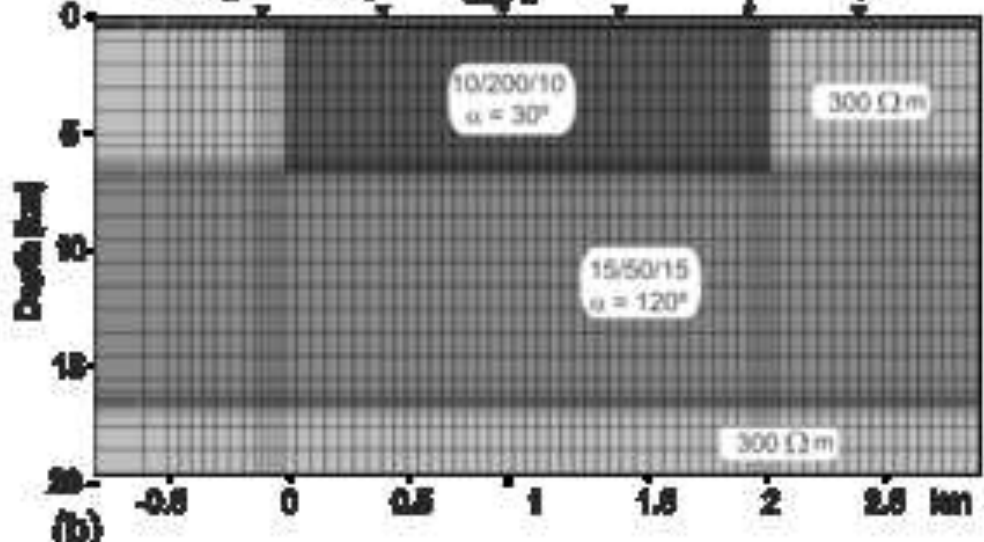

(b) 

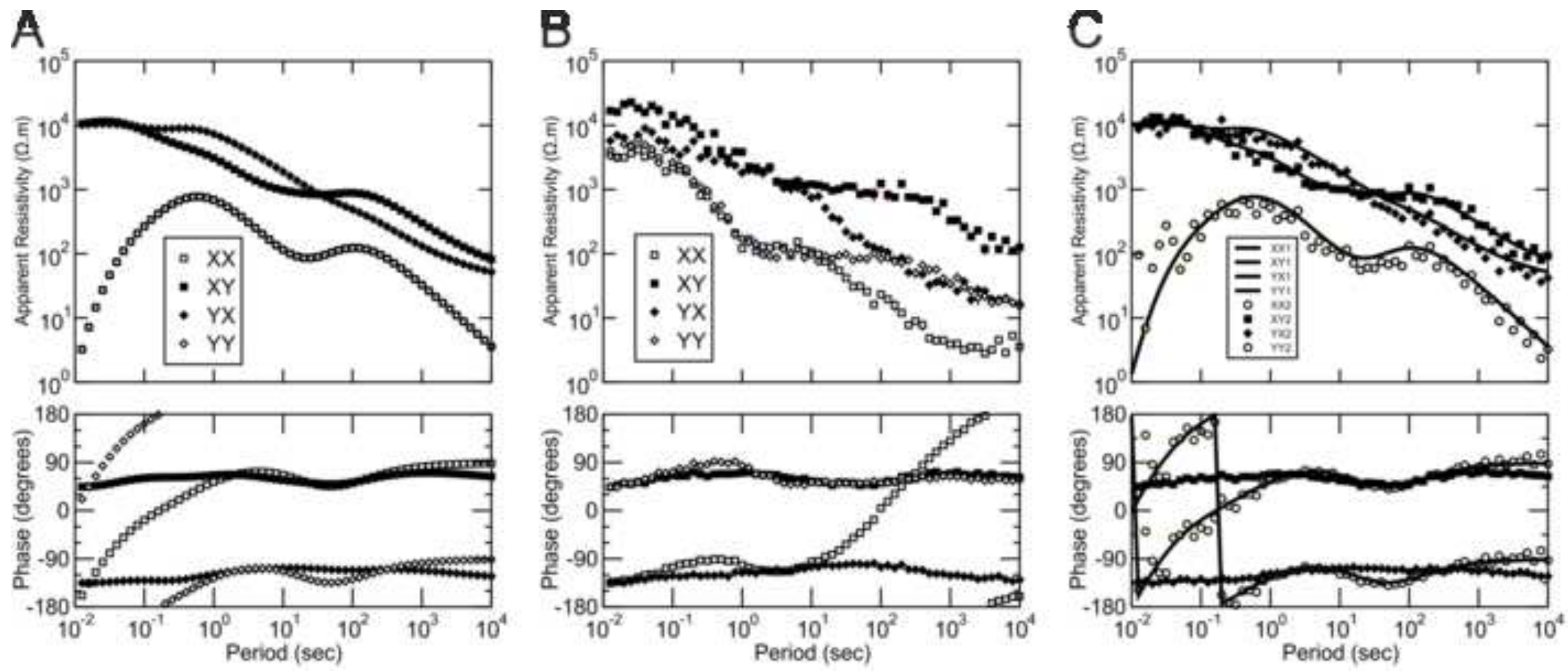

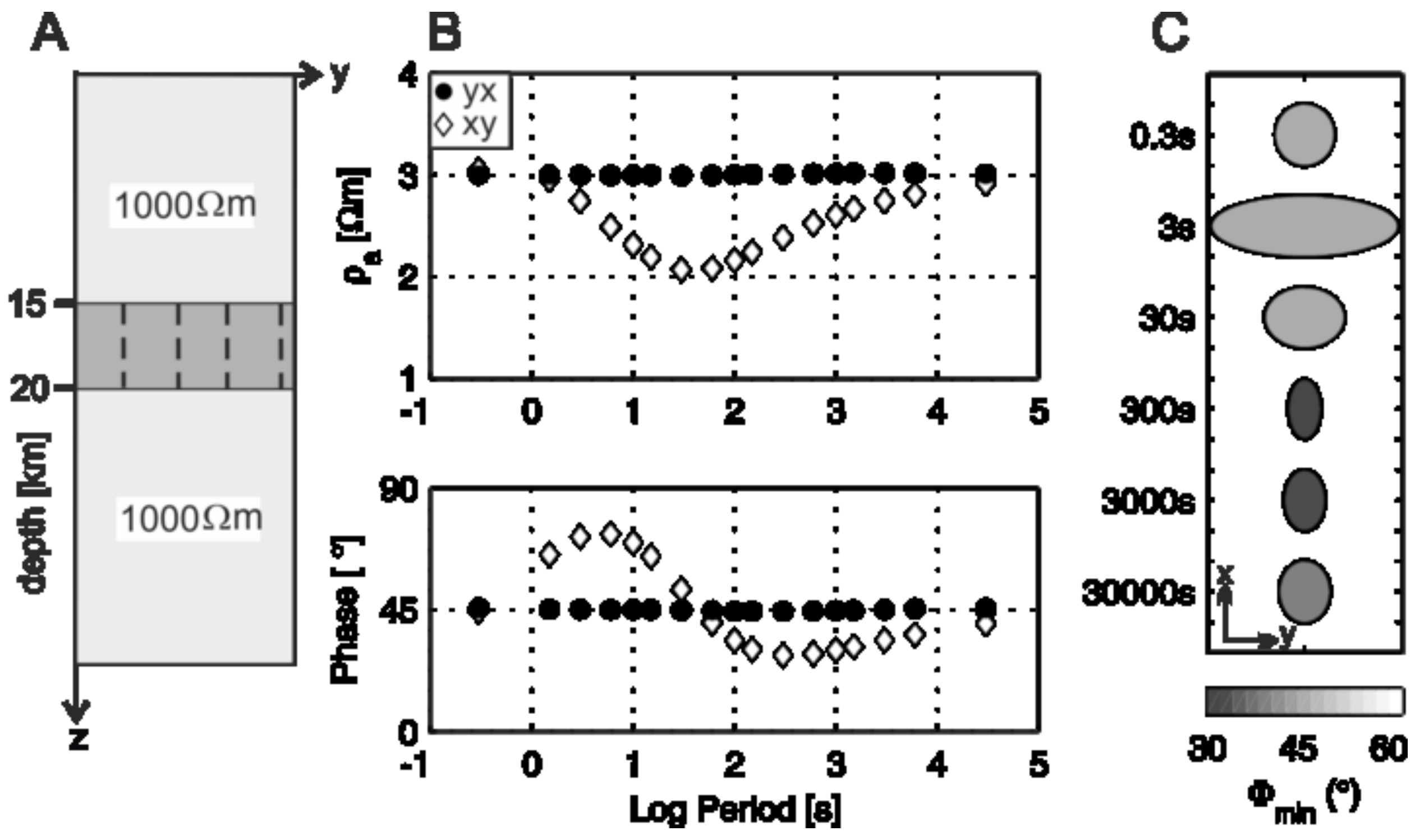


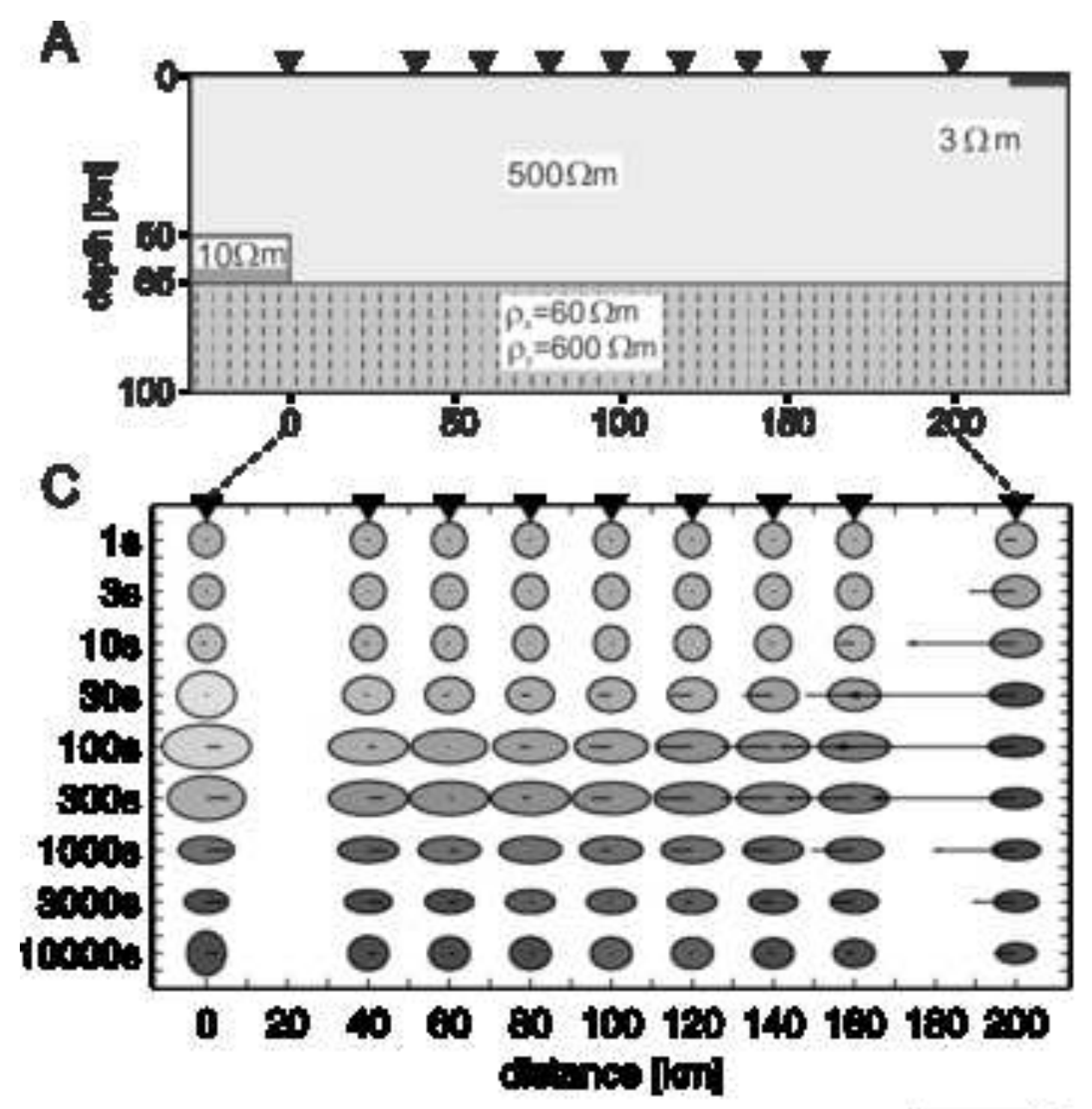

$\overrightarrow{R e(K) 0.6}$

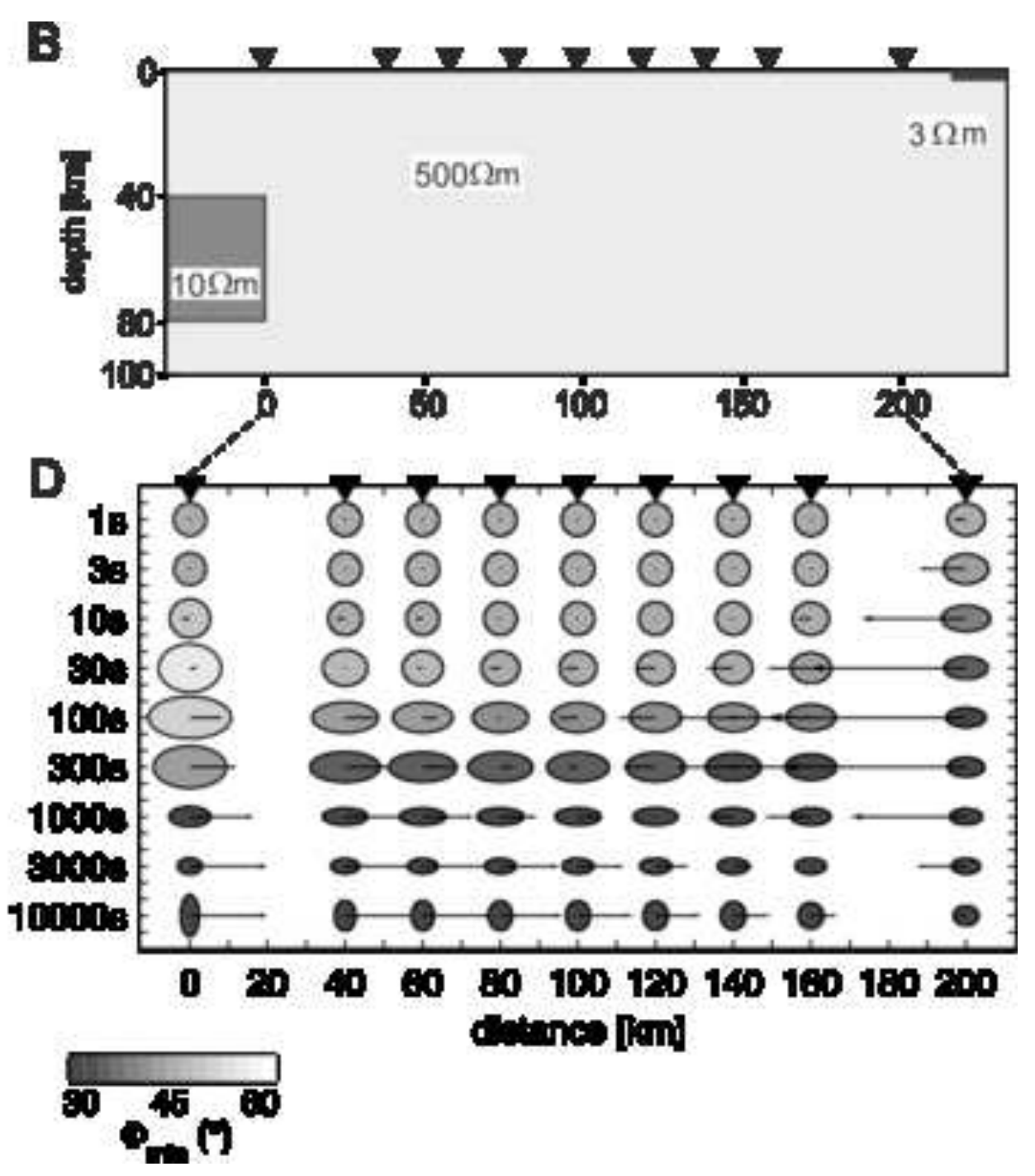




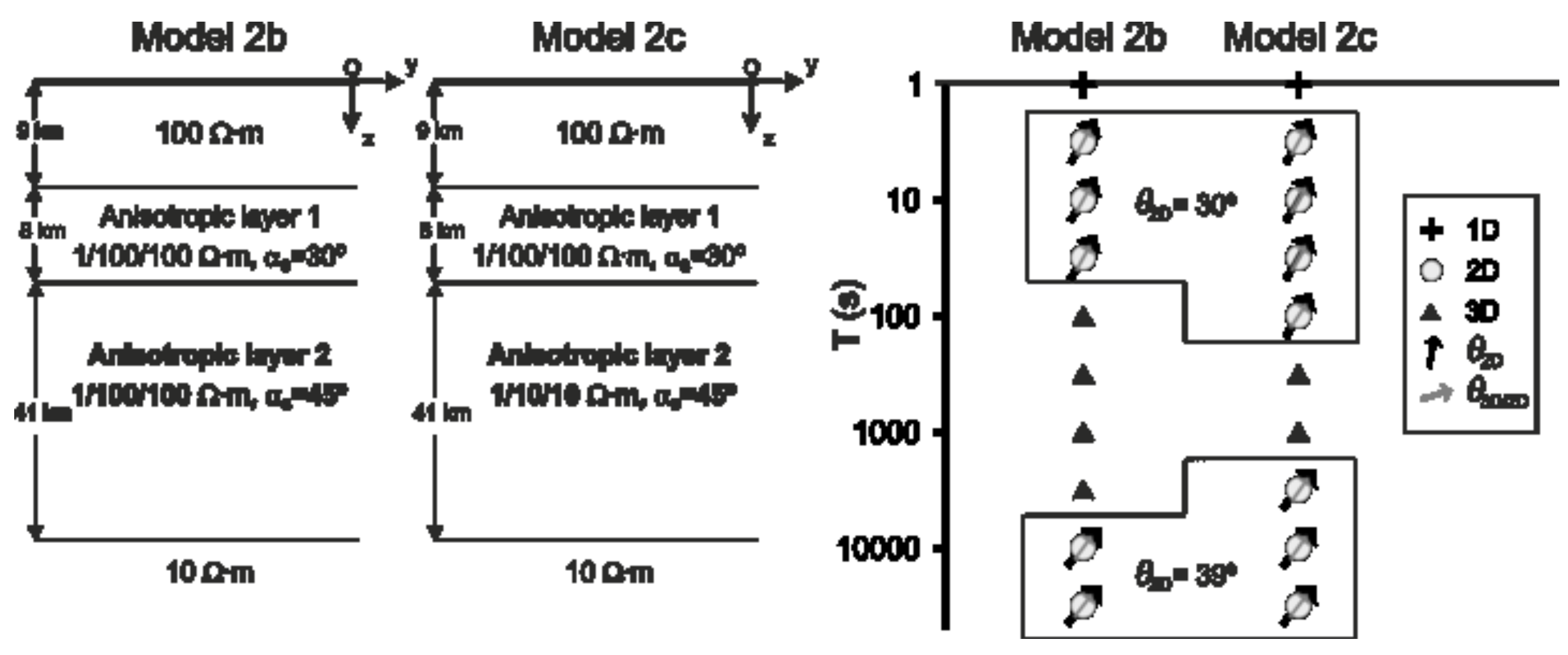



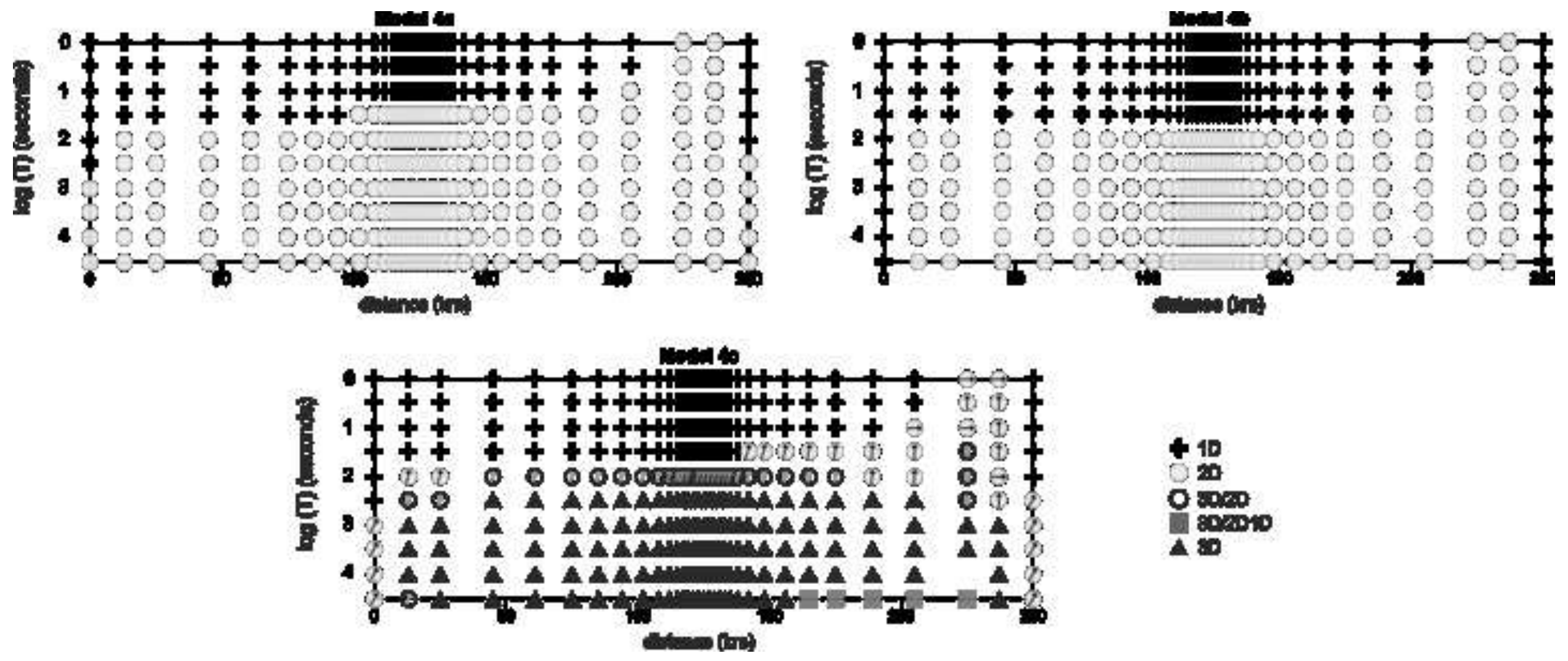

Anas (b) 
Click here to download high resolution image
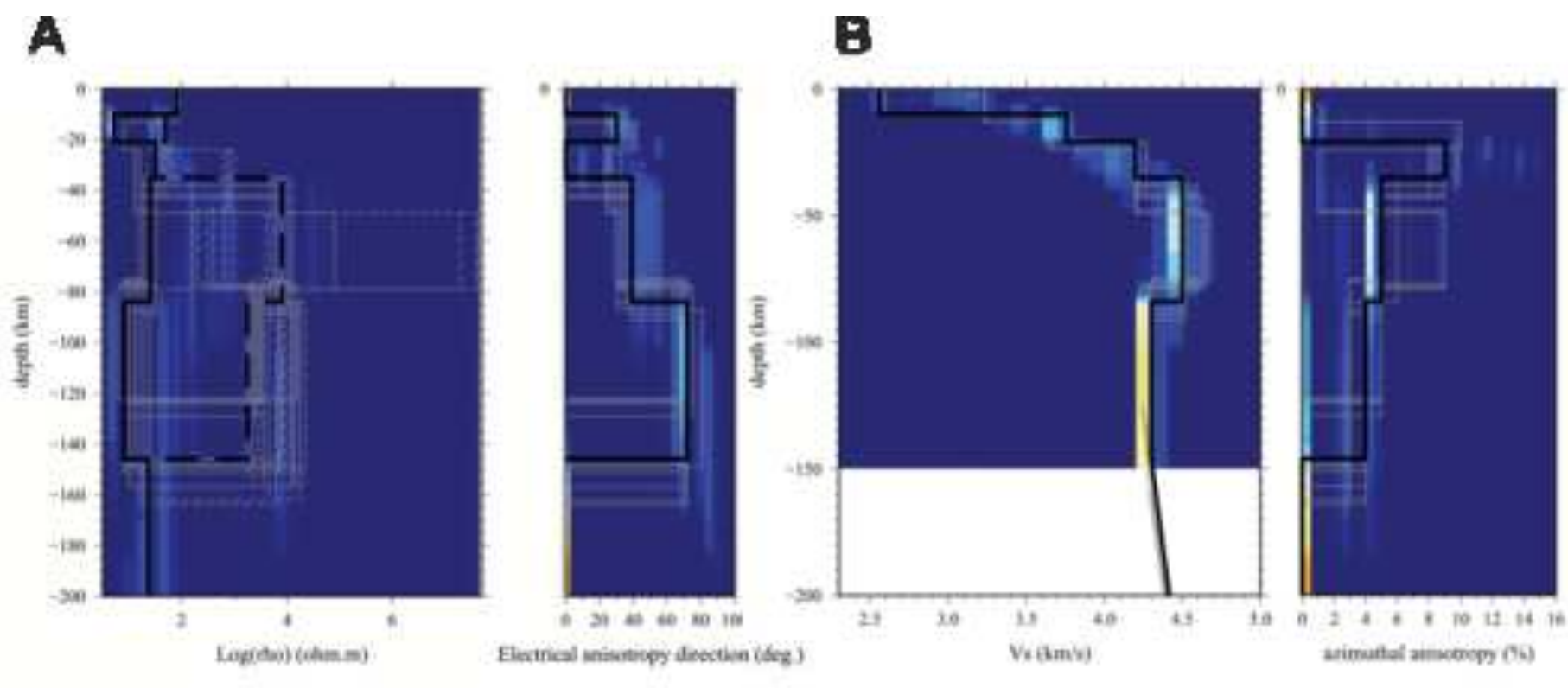

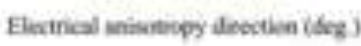

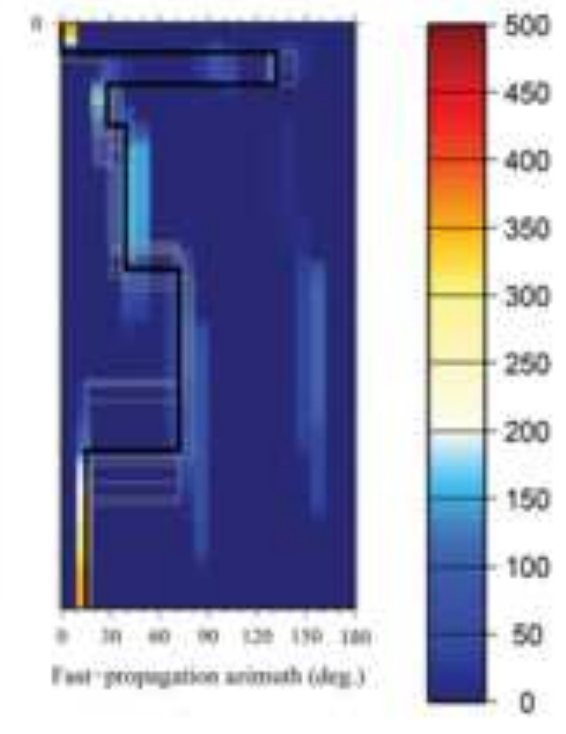

Inet propugation arimath (dey) 

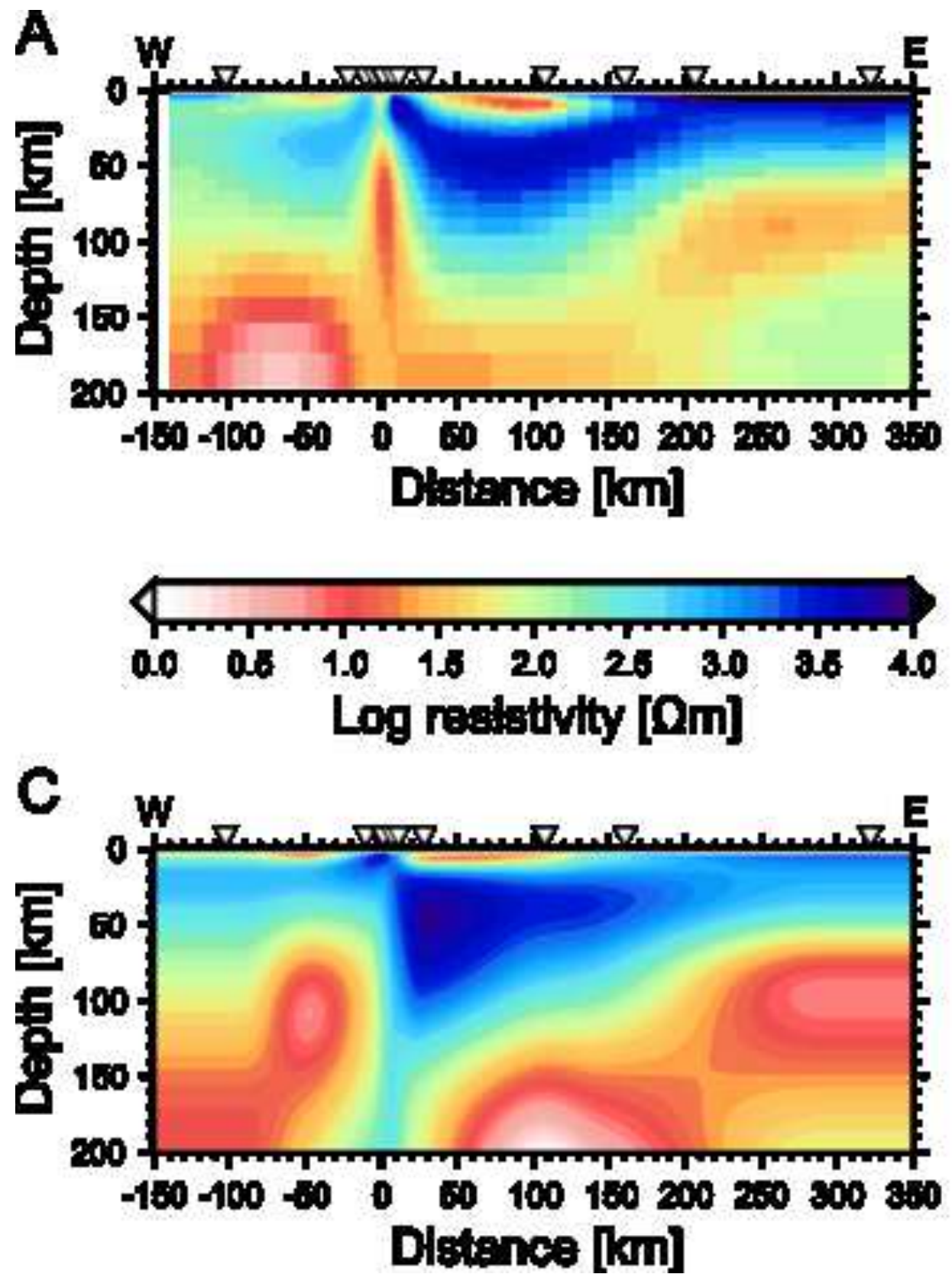
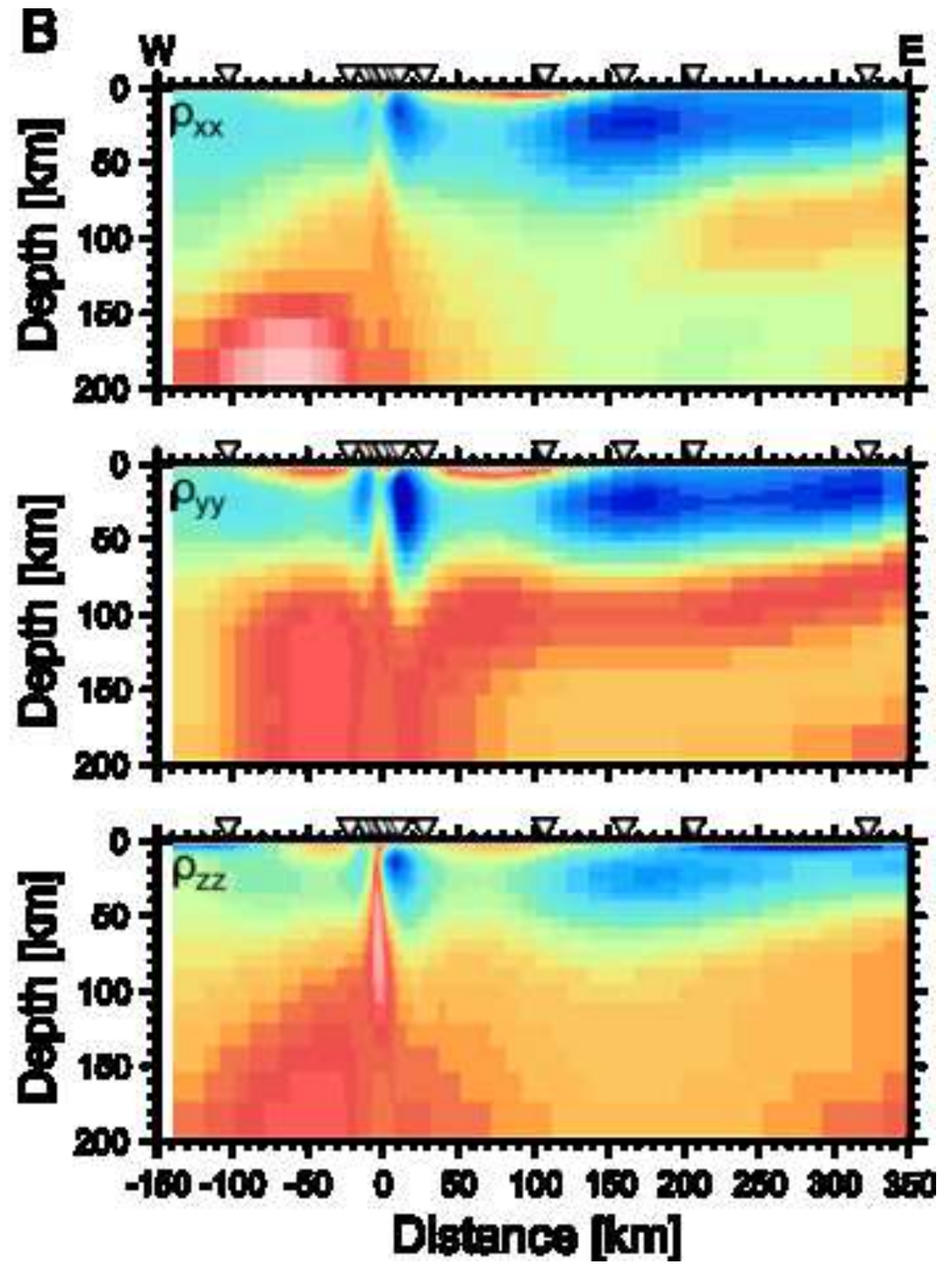

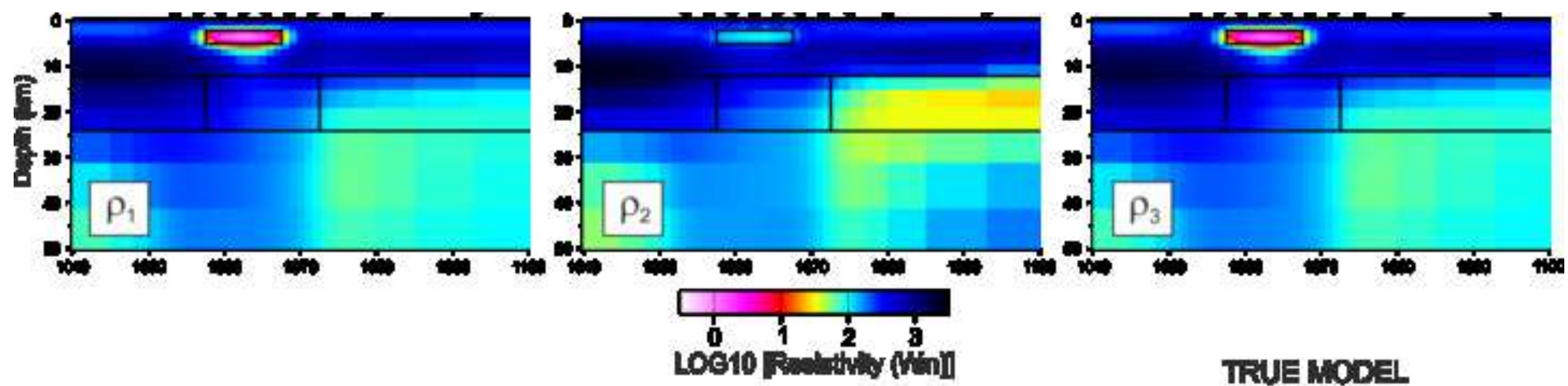

TRUE MODEL
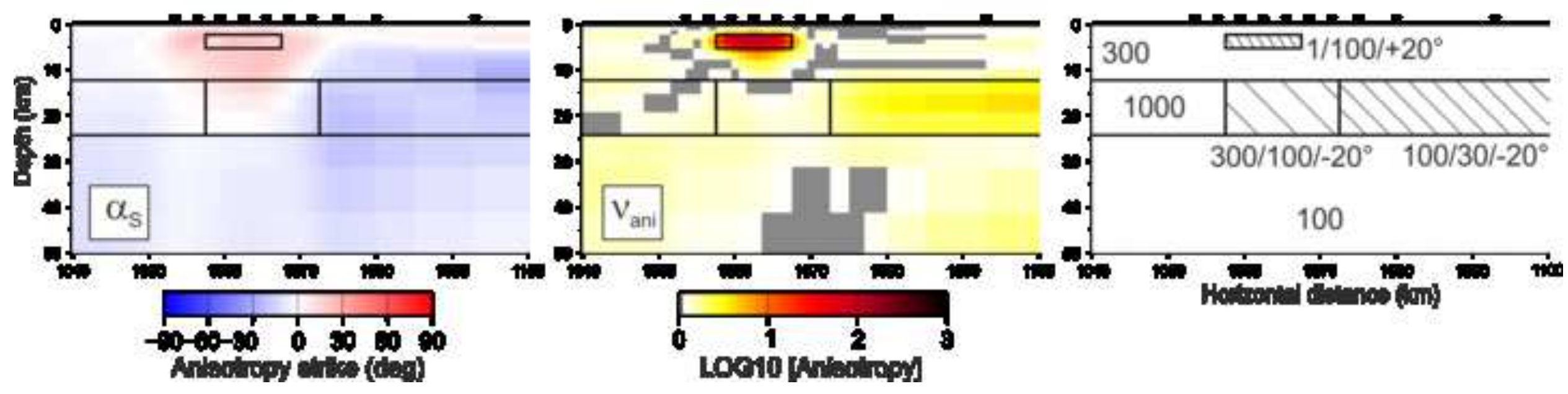

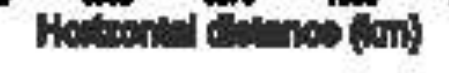




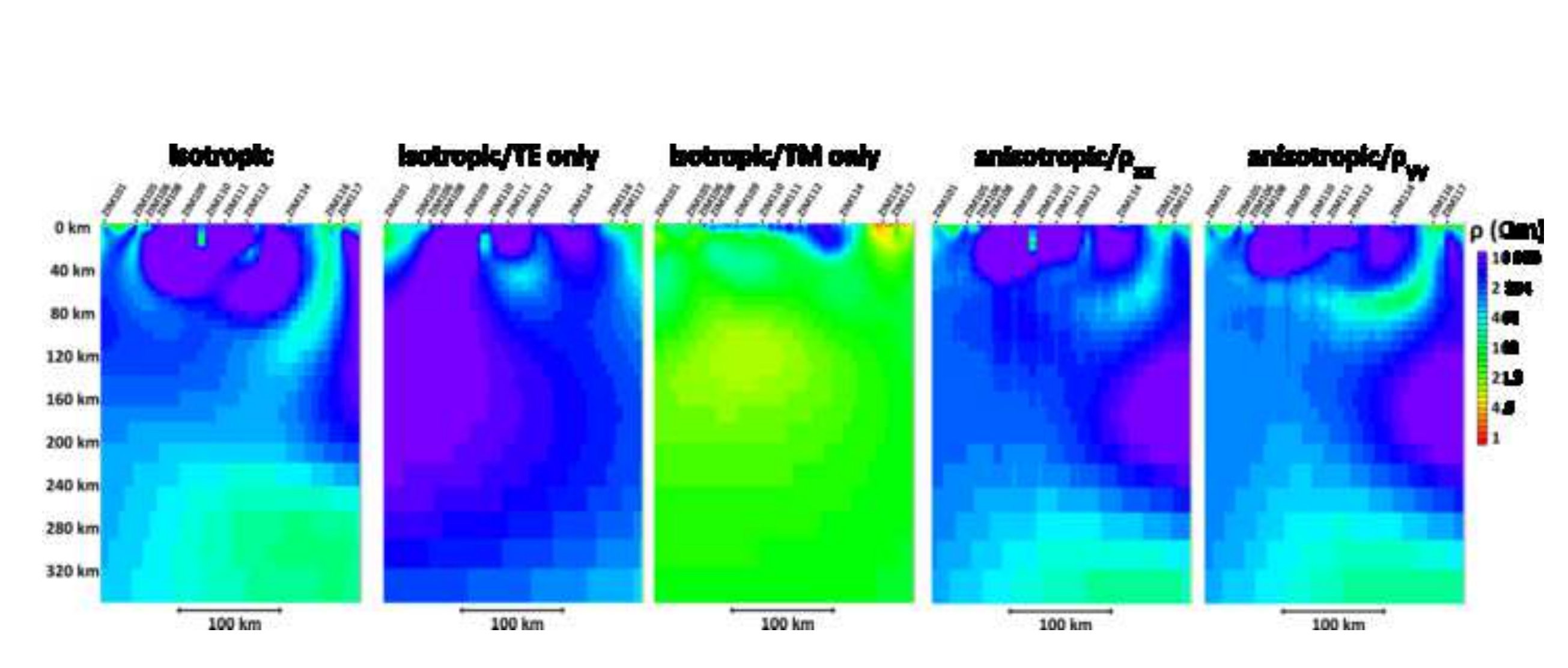

.

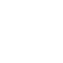

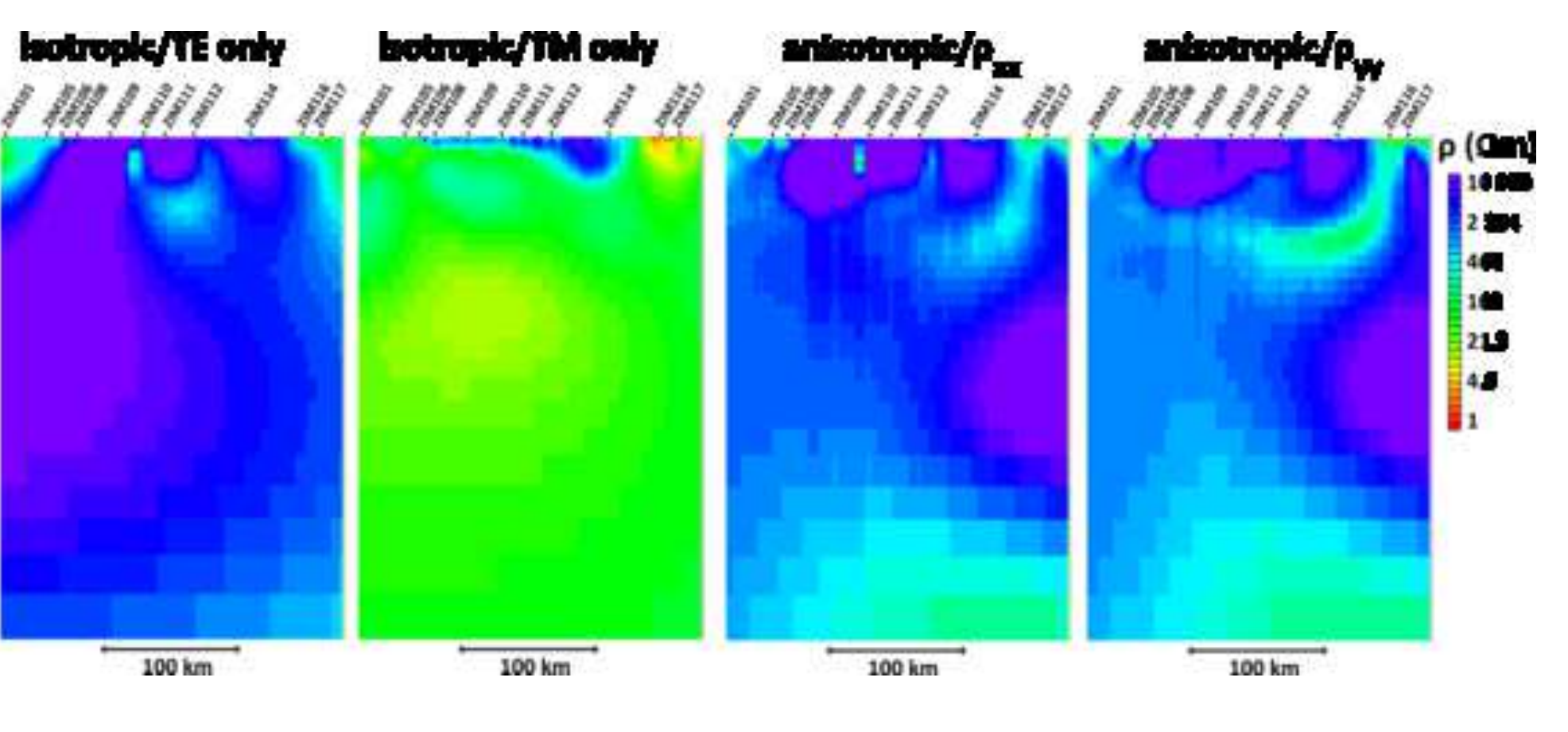




\begin{tabular}{|c|c|c|c|}
\hline$\sigma$ & $\begin{array}{c}\text { AZIMUTHAL } \\
\alpha_{S} \neq 0 \text { and } \\
\alpha_{D}=\alpha_{L}=0\end{array}$ & $\begin{array}{c}\text { DIPPING } \\
\alpha_{D} \neq 0 \text { and } \\
\alpha_{S}=\alpha_{L}=0\end{array}$ & $\begin{array}{c}\text { VERTICAL } \\
\alpha_{S}=\alpha_{D}=\alpha_{L}=0\end{array}$ \\
\hline $\begin{array}{c}\text { BIAXIAL } \\
\left(\sigma_{x} \neq \sigma_{y} \neq \sigma_{z}\right)\end{array}$ & {$\left[\begin{array}{ccc}\sigma_{x x} & \sigma_{x y} & 0 \\
\sigma_{y x} & \sigma_{y y} & 0 \\
0 & 0 & \sigma_{z z}\end{array}\right]$} & {$\left[\begin{array}{ccc}\sigma_{x x} & 0 & 0 \\
0 & \sigma_{y y} & \sigma_{y z} \\
0 & \sigma_{z y} & \sigma_{z z}\end{array}\right]$} & 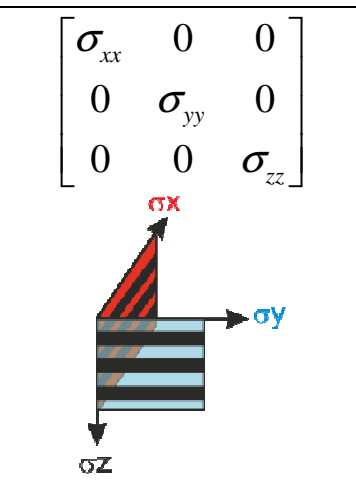 \\
\hline $\begin{array}{l}\text { UNIAXIAL } \\
\text { (and along the } \\
\text { measuring axes) }\end{array}$ & 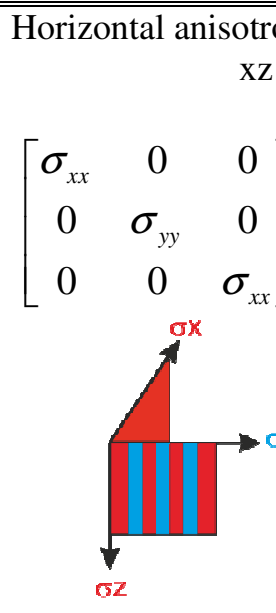 & 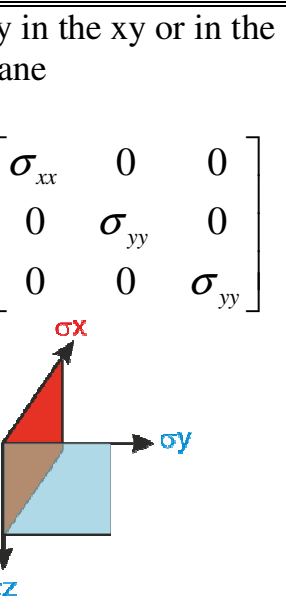 & $\begin{array}{l}\text { Transverse anisotropy in } \\
\text { the vertical direction } \\
{\left[\begin{array}{ccc}\sigma_{h} & 0 & 0 \\
0 & \sigma_{h} & 0 \\
0 & 0 & \sigma_{v}\end{array}\right]} \\
\underset{\sigma v}{\rightarrow}\end{array}$ \\
\hline
\end{tabular}


Click here to download table: Table2_Marti_2012.docx

\begin{tabular}{|c|c|c|c|c|}
\hline$I_{3}$ to $I_{7}, Q$ & \multicolumn{4}{|c|}{ GEOELECTRIC DIMENSIONALITY (“2D cases”) } \\
\hline \multirow{4}{*}{$\begin{array}{l}I_{3} \neq 0 \text { or } I_{4} \neq 0 \\
I_{5}=I_{6}=0 \\
I_{7}=0 \text { or } Q=0\end{array}$} & \multirow[t]{2}{*}{$\theta_{2 D}=\theta_{1}=\theta_{2}=\theta_{3 D / 2 D}$} & $\begin{array}{l}\text { Identical } \\
\text { tensors at } \\
\text { all sites } \\
\text { (period } \\
\text { dependent) }\end{array}$ & $\theta_{2 D} \neq 0$ & $\begin{array}{l}\text { 1D MEDIUM WITH ONE } \\
\text { ANISOTROPIC LAYER or } \\
\text { 2D ISOTROPIC MEDIUM } \\
\text { WITH MEASUREMENTS } \\
\text { ALONG STRIKE } \\
\text { 1D MEDIUM WITH ONE } \\
\text { ANISOTROPIC LAYER }\end{array}$ \\
\hline & & \multicolumn{2}{|c|}{$\begin{array}{l}\text { Different tensors at } \\
\text { each site (period } \\
\text { dependent) }\end{array}$} & 2D ISOTROPIC MEDIUM \\
\hline & $\theta_{2 D} \neq \theta_{3 D / 2 D}$ & \multicolumn{2}{|c|}{$\begin{array}{l}\text { Identical tensors at all } \\
\text { sites (period } \\
\text { independent) }\end{array}$} & $\begin{array}{l}\text { HOMOGENEOUS } \\
\text { ANISOTROPIC MEDIUM }\end{array}$ \\
\hline & $\begin{array}{c}\theta_{2 D} \neq \theta_{3 D / 2 D} \\
\text { or } \\
\theta_{1} \neq \theta_{2}\end{array}$ & \multicolumn{2}{|c|}{$\begin{array}{l}\text { Different tensors at } \\
\text { each site (period } \\
\text { dependent) }\end{array}$} & $\begin{array}{c}\text { ANISOTROPIC } \\
\text { STRUCTURE IN A 2D } \\
\text { MEDIUM }\end{array}$ \\
\hline
\end{tabular}

